\title{
EFEITOS DA DESORDEM DO CAMPO CRISTALINO SOBRE AS FASES MODULADAS DE UM MODELO MAGNÉTICO
}

NELSON ALVES JUNIOR

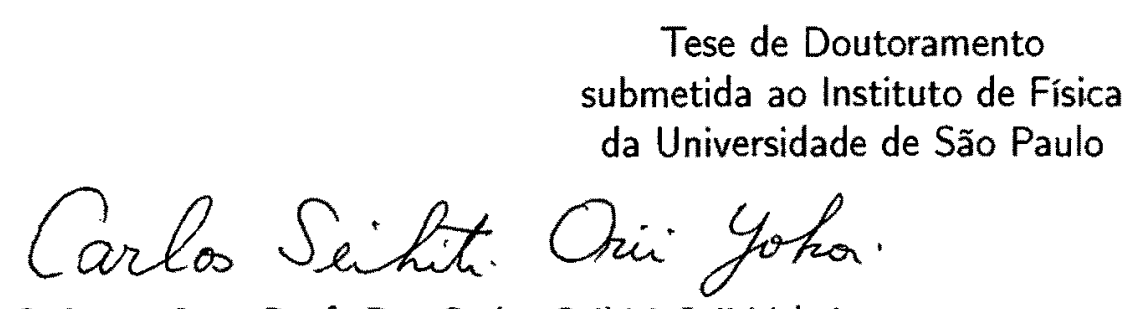

Orientador: Prof. Dr. Carlos Seihiti Orii Yokoi

Banca examinadora:

Prof. Dr. Carlos Seihiti Orii Yokoi (IFUSP)

INSTITUTO DE FÍSICA

Prof. Dr. Evaldo Mendonça Fleury Curado (CBPF)

Serviço de Riblioteca e Informação

Prof. Dr. Silvio Roberto de Azevedo Salinas (IFUSP)

Prof. Dr. Sergio Galvão Coutinho (UFPE)

Tombo: $\frac{3587}{e x \cdot 1}$

Prof. Dr. Valdir Bindilatti (IFUSP)

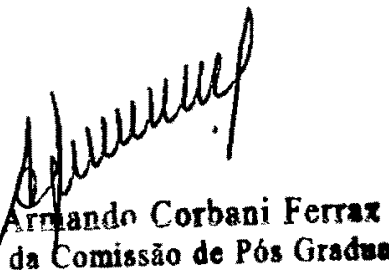
2001

SBI-IFUSP 
FICHA CATALOGRÁFICA

Preparada pelo Serviço de Biblioteca e Informação do Instituto de Física da Universidade de São Paulo

Alves Junior, Nelson

Efeitos da Desordem do Campo Cristalino sobre as Fases Moduladas de um Modelo Magnético.

São Paulo, 2001.

Tese (Doutoramento) - Universidade de São Paulo Instituto de Física - Departamento de Física Geral

Orientador: Prof. Dr. Carlos Seihiti Orii Yokoi Área de Concentração: Fisica do Estado Sólido

Unitermos: 1. Diagramas de Fases; 2. Simulações de Monte Carlo; 3. Campo Cristalino; 4. Sistemas Desordenados; 5 Interações Competitivas. 
Círculo Limite IV (M. C. Escher, 1960)

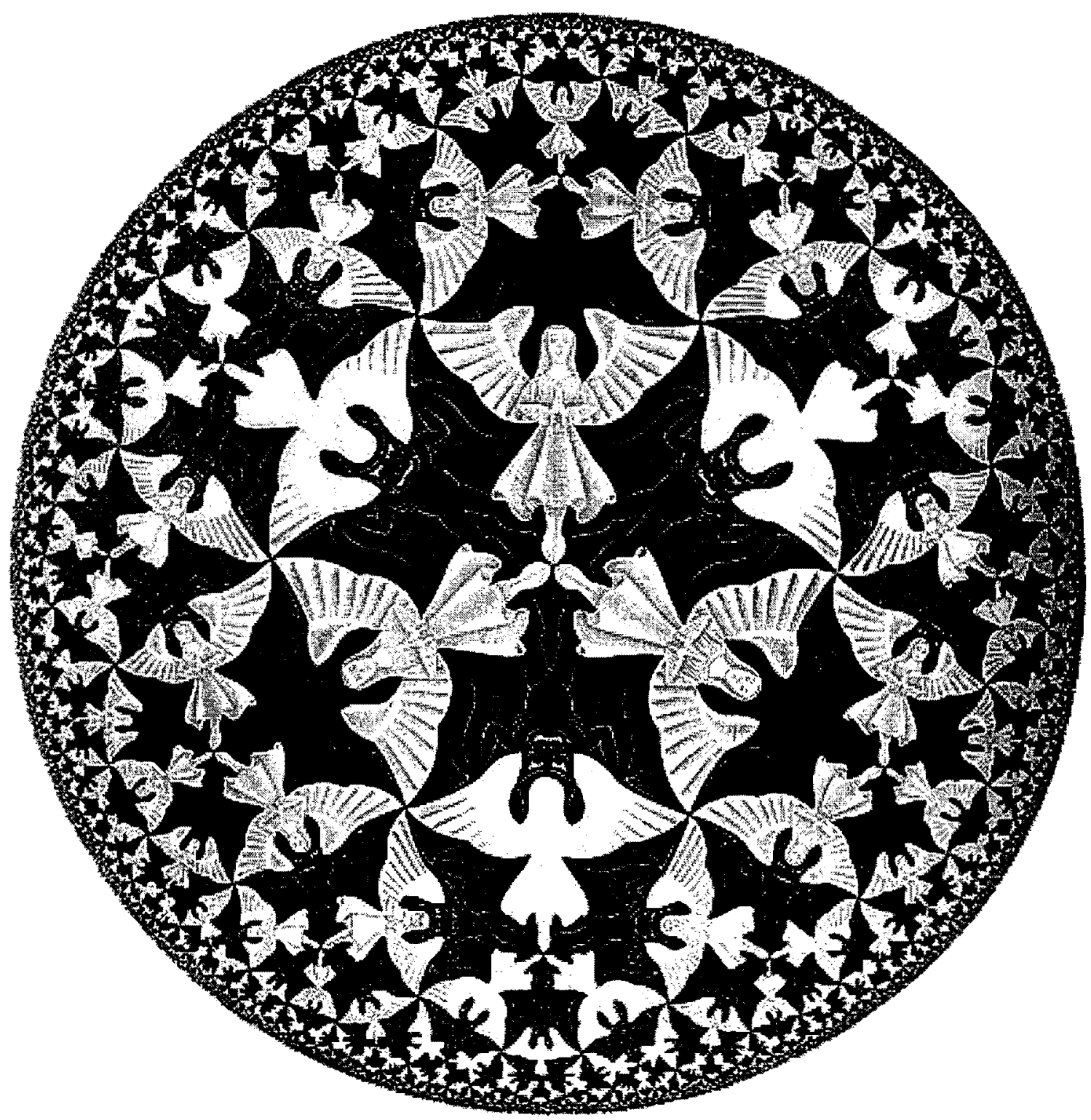

Dedico esta tese à Dualidade 


\section{Agradecimentos}

aos meus pais e à minha irmã, pelo apoio, incentivo e pelo amor que nos une.

ao Carlos Yokoi, pela orientação.

ao Silvio Salinas, pelas primeiras aulas de Mecânica Estatística (bom começo...) e por todos os pedidos de ajuda atendidos.

aos meus grandes amigos Claudia, Ricardo, Mario, Roberto, Karina Barbosa, Endrick e Karina (Birrú), pela lealdade, sinceridade, apoio e amizade, sem os quais nem esta tese, nem tantos momentos da minha vida teriam sentido. aos professores e alunos do Grupo de Mecânica Estatística, por todos os anos de convívio.

às secretárias Márcia, Dirce e Ivone, e ao Alexandre, contínuo do departamento, pela assistência sempre eficiente nos assuntos burocráticos.

aos porteiros João, Marcos, Silvio e Wilson pela amizade e pelas inúmeras madrugadas de solidariedade.

à Universidade de São Paulo.

à FAPESP, pelo apoio financeiro. 


\section{Resumo}

Nesta tese realizamos um estudo de campo médio dos efeitos da desordem do campo cristalino sobre os diagramas de fases do modelo ANNNI com spin 1.

Resultados de campo médio anteriores obtidos para o modelo com campo cristalino constante mostraram fases parcialmente desordenadas em seus diagramas de fases. No entanto, a existência de tais fases não é garantida, devido às flutuações não levadas em conta na aproximação de campo médio. Sendo assim, estudamos o modelo com campo cristalino constante por meio de simulações de Monte Carlo. Nosso objetivo foi fornecer maior credibilidade aos resultados de campo médio obtidos anteriormente, e com isso adquirirmos maior confiança nos resultados de campo médio apresentados nesta tese para um campo cristalino aleatório. Os resultados das simulações mostraram evidências de que a configuração com planos desordenados da fase comensurável de período 6, observada em baixas temperaturas, pode sofrer uma transição interna para uma configuração na qual tais planos estão ausentes. Esse resultado está em concordância com os estudos de campo médio.

Para o caso de um campo cristalino aleatório, investigamos os diagramas de fases do modelo na aproximação de campo médio. Para uma distribuição de probabilidades genérica do campo cristalino, obtivemos o funcional da 
energia livre e as equações de campo médio, assim como as expressões das linhas críticas e dos pontos tricríticos do modelo. Para investigarmos os efeitos da desordem sobre os diagramas de fases, consideramos as distribuições de probabilidade do campo cristalino delta-bimodal e gaussiana. 


\section{Abstract}

In this thesis we present a mean-field study of the effects of the disorder of the single-ion anisotropy on the phase diagrams of the spin-1 ANNNI model.

Previous mean-field results for the model with constant single-ion anisotropy showed partially disordered phases in the phase diagrams of the model. However, the existence of such phases cannot be taken as granted, due to the fluctuations not taken into account in the mean-field approximation. Thus, we studied the model with constant single-ion anisotropy by means of Monte Carlo simulations. Our aim was to give credit to previous mean-field results and to get more confidence in the mean-field results showed in this thesis obtained for a random single-ion anisotropy. The results obtained from the simulations showed evidences that the configuration of the commensurate phase with period 6 displaying disordered planes, found at low temperatures, may undergo an internal phase transition to a phase in which such planes are absent. This result is in agreement with previous mean-field studies.

For a random single-ion anisotropy, we investigated the phase diagrams of the model within mean-field approximation. For a generic probability distribution of the anisotropy, we obtained the free-energy functional and the mean-field equations, as well as the expressions for the critical lines and tricritical points of the model. In order to investigate disorder effects, we obtained 
phase diagrams of the model by considering a double-delta and a Gaussian probability distribution of the single-ion anisotropy. 


\section{Conteúdo}

1 Introdução 1

2 Caso sem desordem: Simulações de Monte Carlo 13

2.1 Introdução . . . . . . . . . . . . . . . . . 13

2.2 Resultados das simulações . . . . . . . . . . . 16

3 Caso com desordem: Aproximação de campo médio 32

3.1 Introdução . . . . . . . . . . . . . . . . . 32

3.2 Equações de campo médio e funcional da energia livre . . . . . 35

$3.3 \quad$ O limite $T \rightarrow 0 \ldots \ldots \ldots \ldots$

3.4 Expansão de Landau . . . . . . . . . . . . . . . . . . . . . 40

3.5 Linhas críticas e pontos tricríticos . . . . . . . . . . . 42

4 Resultados para uma distribuição delta-bimodal 45

4.1 Diagramas de fases em $T=0 \ldots \ldots$. . . . . . . . 46

4.2 Linhas críticas e pontos tricríticos . . . . . . . . . . 66

4.3 Diagramas de fases para $T>0 \ldots \ldots 70$

4.4 O modelo ANNNI com spin $1 / 2$ e diluição de sítios . . . . . . 79

5 Resultados para uma distribuição gaussiana 86 
5.1 Diagramas de fases em $T=0 \ldots \ldots$. . . . . . 87

5.2 Linhas críticas e pontos tricríticos . . . . . . . . . . . 98

5.3 Diagramas de fases para $T>0 \ldots . \ldots 102$

6 Considerações finais 106

A Expansão de Landau para uma distribuição de probabilidades genérica do campo cristalino

B Determinação do ponto crítico em $T=0$ para uma distribuição gaussiana do campo cristalino 


\section{Capítulo 1}

\section{Introdução}

Sistemas espacialmente modulados são caracterizados por exibirem propriedades locais que variam periodicamente em uma ou mais direções espaciais (Selke, 1992). Diferentes ordens moduladas foram observadas em inúmeros sistemas em Matéria Condensada. Por exemplo, materiais magnéticos (Izyumov, 1984), gases nobres adsorvidos em substratos cristalinos (Stephens et al., 1984), compostos ferroelétricos (Yamada, Hoshino, e Shibuya, 1963; Iizumi, Axe, Shirane, e Shimaoka, 1977), ondas de densidades de carga (Comes, Shapiro, Shirane, Garito, e Heeger, 1975), compostos intercalados de grafite (Fischer e Thompson, 1978) e cristais líquidos (Gennes, 1974). Uma fase modulada é dita comensurável se a razão entre a periodicidade da modulação e o parâmetro da rede for um número racional, e incomensurável caso contrário. O mecanismo básico para a ocorrência da modulação é a competição entre interações favorecendo ordenamentos distintos, o que dá origem ao fenômeno conhecido como frustração (Toulouse, 1977). Existe uma classificação segundo a qual os sistemas modulados podem ser agrupados em cinco categorias (Cummins, 1990): modulação por deslocamento, por orientação, por composição, por crescimento interno e modulação interfacial. Essas cinco categorias 
não são exclusivas, ou seja, um mesmo material pode apresentar mais de um tipo de modulação. Por exemplo, o modelo magnético estudado neste trabalho pode ser classificado como um sistema com modulação por orientação, por crescimento interno e interfacial. Com a descoberta da difração de nêutrons na década de 50, as estruturas moduladas puderam ser observadas pela primeira vez em metais terras-raras (lantanídeos) (Cooper, 1968). A estrutura magnética do hólmio foi estudada com difração de nêutrons (Koehler, Cable, Child, Wilkinson, e Wollan, 1966, 1967; Felcher, Lander, Arai, Sinha, e Spedding, 1976; Pechan e Stassis, 1984). Em temperaturas suficientemente baixas essa estrutura é uma espiral incomensurável com o vetor de onda de propagação na direção perpendicular aos planos basais da estrutura hexagonal compacta. Estudos empregando difração de raios X sincrotron do hólmio e do érbio (Bohr, Gibbs, Moncton, e Damico, 1986; Gibbs, Bohr, Axe, Moncton, e Damico, 1986) mostraram que o vetor de onda desses materiais varia com a temperatura, como mostrado nas figuras 1.1 e 1.2. Os platôs observados nessas figuras correspondem ao fenômeno de ancoramento (lock-in) das fases comensuráveis.

No final dos anos 50 as estruturas moduladas em terras-raras foram interpretadas (Villain, 1959; Kaplan, 1959; Yoshimori, 1959; Elliott, 1961) como sendo a manifestação da competição proveniente da interação de troca espacialmente oscilatória do tipo RKKY (Ruderman e Kittel, 1954; Kasuya, 1956; Yosida, 1957), existente entre os elétrons $4 \mathrm{f}$ e mediada pelos elétrons de condução.

Do ponto de vista teórico, diversos modelos com interações competitivas 


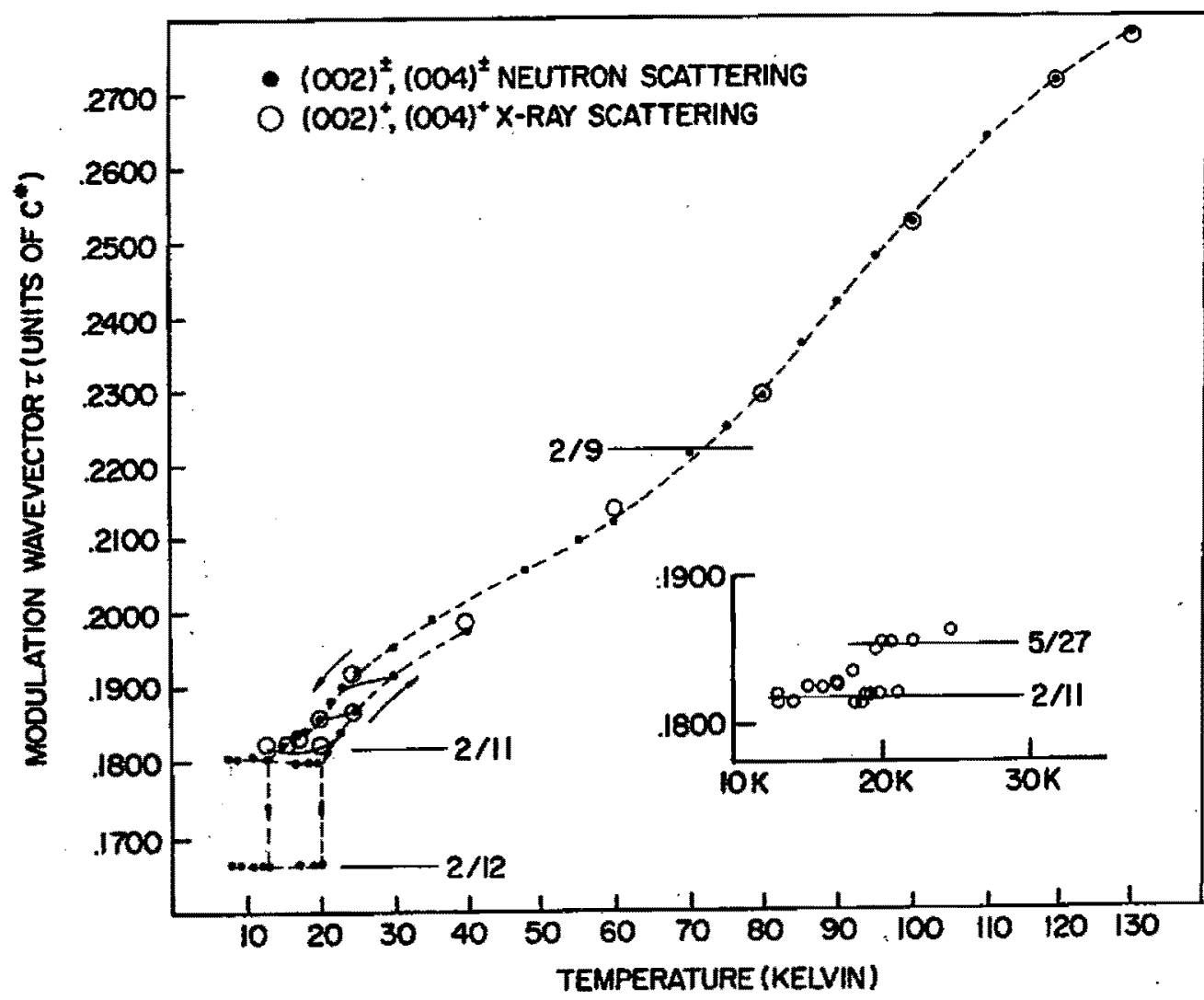

Figura 1.1: Dependência do vetor de onda magnético do hólmio com a temperatura, conforme obtida com difração de raios $\mathrm{X}$ de sincrotron (círculos vazios) ou com difração de nêutrons (pontos). $\left(\boldsymbol{a}^{*}, \boldsymbol{b}^{*}, \boldsymbol{c}^{*}\right.$ são os geradores da rede recíproca da rede hcp gerada por $\boldsymbol{a}, \boldsymbol{b}, \boldsymbol{c}$. $\boldsymbol{c}^{*}$ é paralelo a $\boldsymbol{c}$, que por sua vez é perpendicular aos planos basais). [Bohr e Gibbs, 1986].

foram propostos e investigados, mesmo antes que as estruturas moduladas pudessem ser observadas experimentalmente (Nagamiya, 1967; Cooper, 1968; Elliott, 1972). A seguir apresentamos alguns modelos mecânico-estatísticos para sistemas modulados, com o objetivo principal de ilustrar os diferentes mecanismos de competição responsáveis pelo surgimento da modulação.

Um modelo prototípico apresentando um complexo diagrama de fases moduladas comensuráveis e incomensuráveis consiste de uma cadeia de átomos ligados a seus primeiros vizinhos por molas e sujeitos a um potencial externo 


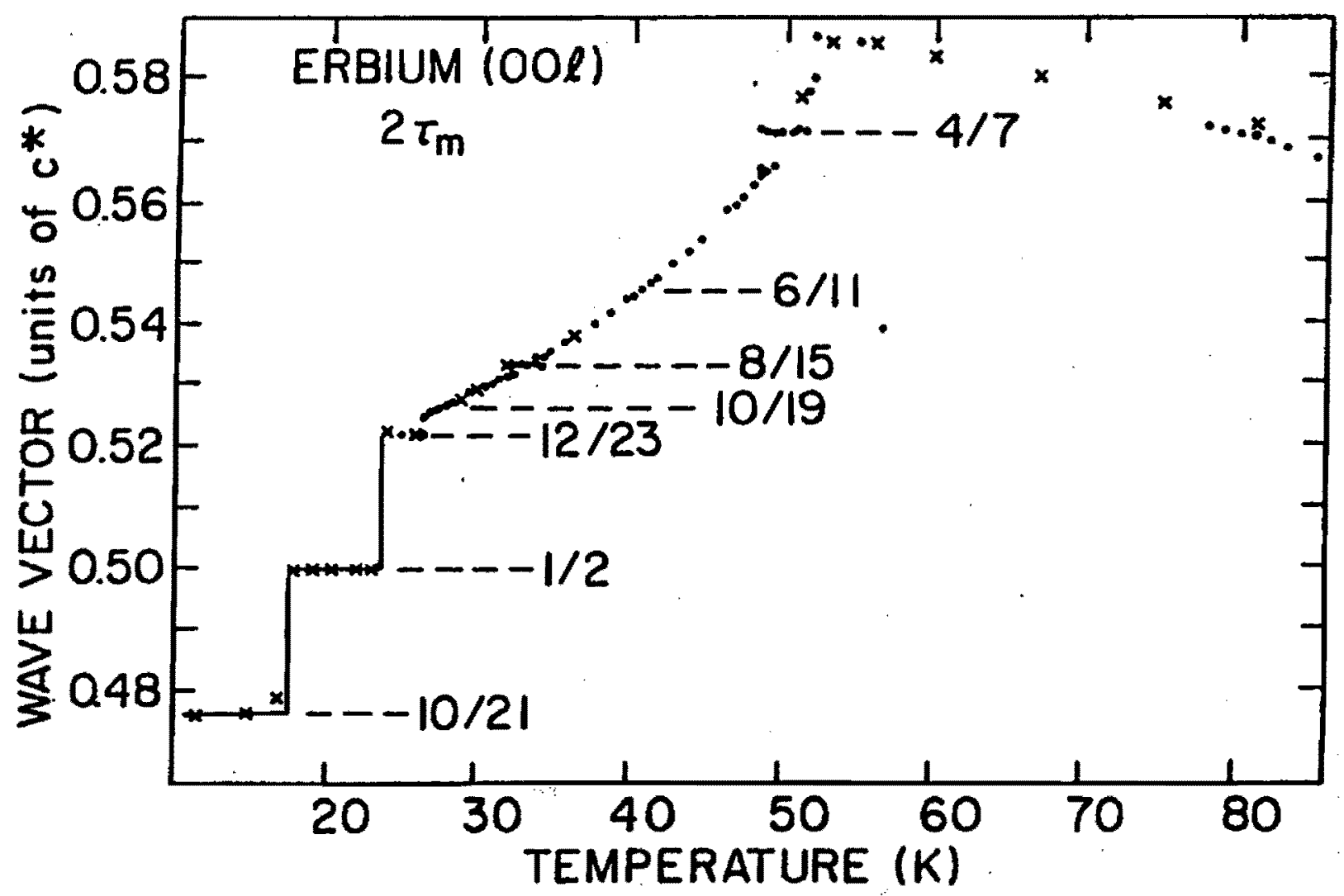

Figura 1.2: Dependência do vetor de onda magnético do érbio com a temperatura, conforme obtida com difração de raios $\mathrm{X}$ de sincrotron (pontos) ou com difração de nêutrons (cruzes). $\left(\boldsymbol{a}^{*}, \boldsymbol{b}^{*}, \boldsymbol{c}^{*}\right.$ são definidos como na figura 1.1). [Gibbs, Bohr e Axe, 1986].

periódico, conhecido como modelo de Frenkel-Kontorova (FK) (Selke, 1992; Griffiths, 1990). Entre os sistemas físicos que podem ser representados pelo modelo FK, podemos citar aquele em que os átomos de um gás são adsorvidos em um substrato cristalino. As posições de equilíbrio dos átomos estão intimamente ligadas à competição entre as interações elásticas e o potencial externo. Esse mecanismo de competição é responsável pela modulação observada no modelo FK. Embora o modelo tenha sido proposto no final da década de 20 , foi apenas no final dos anos 70 que estudos mais aprofundados foram realizados, especialmente sobre as propriedades dos diagramas de fases no zero 
absoluto de temperatura (Aubry, 1978).

Outro modelo apresentando estruturas moduladas em seus diagramas de fases é o modelo XY quiral, formado por uma série de cadeias de spins planares, dispostas paralelamente umas às outras e perpendicularmente ao plano dos spins. Dentro de cada plano XY os spins primeiros vizinhos interagem ferromagneticamente. Sendo assim, no zero absoluto de temperatura as cadeias estão identicamente ordenadas. Por outro lado, ao longo das cadeias as interações entre spins primeiros vizinhos favorecem o ordenamento helicoidal, no qual spins consecutivos formam um ângulo $\Delta \neq 0$ entre eles. Pode-se adicionar um campo magnético ao modelo. Na presença de um campo magnético paralelo ao plano $\mathrm{XY}$, todos os spins tendem a ordenar-se paralelamente ao campo, ou seja, todos os spins tendem a ficar paralelos uns aos outros. A competição entre esses dois ordenamentos distintos leva ao surgimento de um complexo diagrama de fases espacialmente moduladas (Yokoi, Tang, e Chou, 1988). Efeitos de anisotropia cristalina são comuns em sistemas magnéticos reais. O estudo de tais efeitos pode ser realizado pela adição de um termo de campo cristalino $K$, favorecendo $p$ direções espacias dadas pelos ângulos $\theta_{n}=2 \pi n / p \quad(n=1,2, \cdots, p)$. Na ausência de um campo magnético, o mecanismo responsável pelo surgimento das fases moduladas do modelo é a competição entre as interações quirais e o termo de anisotropia. Para valores suficientemente grandes do termo de anisotropia $(K \rightarrow \infty)$, nesses diagramas foi observada a existência de um ponto multicrítico, denominado ponto $\Upsilon$ (Micheletti, Seno, e Yeomans, 1995; Sasaki, 1992), a partir do qual surgem infinitas fases moduladas. No limite $K=\infty$ o modelo XY quiral reduz-se ao 
modelo de relógio quiral de $p$ estados, ou $\mathrm{CC}_{p}$ ( $p$-state chiral clock) (Ostlund, 1981; Huse, 1981), no qual as variáveis de spin podem ocupar apenas as $p$ posições $\theta_{n}=2 \pi n / p(n=1,2, \cdots, p)$.

No início da década de 60 foi introduzido um modelo (Elliott, 1961), posteriormente denominado por Fisher e Selke de modelo ANNNI (Axial NextNearest-Neighbor Ising) (Fisher e Selke, 1980), que é um dos modelos mais simples capaz de exibir um complexo diagrama de fases espacialmente moduladas (Bak, 1982; Selke, 1988; Yeomans, 1988; Selke, 1992). É definido numa rede cúbica simples cujos sítios são ocupados por variáveis de Ising. Há interações interplanares $J_{1}$ e $J_{2}$ entre primeiros e segundos vizinhos, respectivamente, ao longo do eixo axial $z$, e interações intraplanares $J_{0}$ entre primeiros vizinhos nos planos perpendiculares ao eixo $z$. Uma representação esquemática do modelo ANNNI é mostrada na figura 1.3.

No caso em que $J_{1}$ e $J_{2}$ têm sinais opostos, a competição entre essas interações pode dar origem às fases moduladas. Empregando a aproximação de campo médio Elliott mostrou que a fase paramagnética pode tornar-se instável, dando origem às fases moduladas caracterizadas pelo número de onda (Elliott, 1961)

$$
q=\cos ^{-1}\left(-\frac{J_{1}}{4 J_{2}}\right)
$$

O modelo possui solução exata apenas em uma dimensão, tendo sido estudado tanto na ausência de um campo magnético (Stephenson, 1970a, 1970b) quanto na sua presença (Rujan, Selke, e Uimin, 1983; Bhattacharyya e Dasgupta, 1991; Alves Jr. e Yokoi, 2000), e no zero absoluto de temperatura (Morita e Horiguchi, 1972; Yokoi, Coutinho-Filho, e Salinas, 1981). 


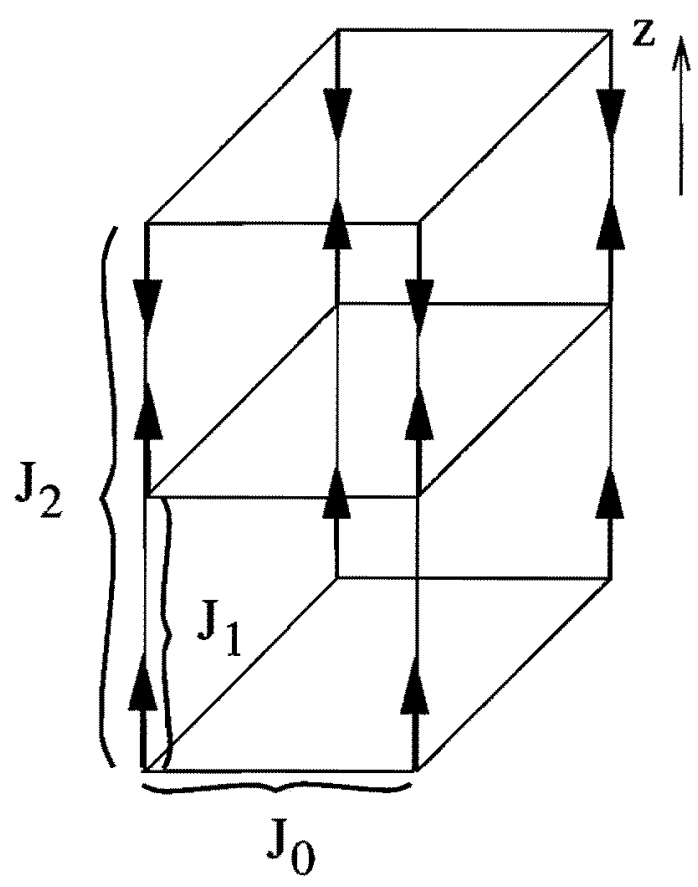

Figura 1.3: Representação do modelo ANNNI.

Embora o modelo tenha sido proposto para explicar a modulação magnética longitudinal observada no érbio (Elliott, 1961), sua gama de aplicações ultrapassa essa motivação original. Entre tais aplicações, podemos citar sistemas ferroelétricos $\left(\mathrm{NaNO}_{2}\right)$, ligas binárias $\left(\mathrm{Ag}_{3} \mathrm{Mg}, \mathrm{CuAu}, \mathrm{Au}_{3} \mathrm{Zn}, \mathrm{TiAl}_{3}\right.$, $\mathrm{Cu}_{3} \mathrm{Pd}$ ), politipos ( $\mathrm{SiC}$ e $\mathrm{CdI}_{2}$ ) e compostos magnéticos (CeSb e terras-raras) (Selke, 1988; Yeomans, 1988). Apesar de todo esse potencial de aplicações, o modelo permaneceu adormecido até meados da década de setenta quando novos desenvolvimentos teóricos incentivaram seu estudo mais aprofundado. O diagrama de fases de campo médio do modelo foi obtido pela primeira vez 
no início dos anos 80, quando foram determinadas as principais fases comensuráveis (Bak e Boehm, 1980). Nesse diagrama de fases foi localizado o Ponto de Lifshitz (Hornreich, Luban, e Shtrikman, 1975), situado no encontro da linha crítica, separando a região paramagnética das regiões ferromagnética e modulada, com a linha de primeira ordem, separando a fase ferromagnética da região modulada (Redner e Stanley, 1977). O ponto de Lifshitz foi observado experimentalmente pela primeira vez no composto magnético $\mathrm{MnP}$ (monofosfeto de manganês) (Becerra, Shapira, Oliveira Jr., e Chang, 1980) e pertence à mesma classe de universalidade do ponto de Lifshitz presente no modelo ANNNI. Além disso, o complexo ordenamento das fases magnéticas comensuráveis observadas no composto CeSb (Rossat-Mignod et al., 1977; Rossat-Mignod, Burlet, Bartholin, Vogt, e Lagnier, 1980) (figura 1.4) foram estudadas teoricamente a partir de um modelo semelhante ao modelo ANNNI. (Boehm e Bak, 1979). Posteriormente, a teoria de campo médio do modelo ANNNI foi melhor explorada por Selke e Duxbury (Duxbury e Selke, 1983; Selke e Duxbury, 1984). Esses autores mostraram que as fases moduladas surgem a partir de um processo de ramificação por combinação de estruturas, segundo o qual, dadas duas fases com números de onda $P / Q$ e $P^{\prime} / Q^{\prime}$, a fronteira entre elas é desestabilizada pelo surgimento de uma fase com número de onda $\left(P+P^{\prime}\right) /\left(Q+Q^{\prime}\right)$ (Selke e Duxbury, 1984). Na figura 1.5 apresentamos o diagrama de fases mais detalhado do modelo ANNNI, construído segundo o método de ramificação proposto por Selke e Duxbury. Sendo assim, durante a década de 80 o interesse pelo modelo ANNNI foi intensamente retomado. Muitos trabalhos foram desenvolvidos com o intuito de mostrar que o modelo, 


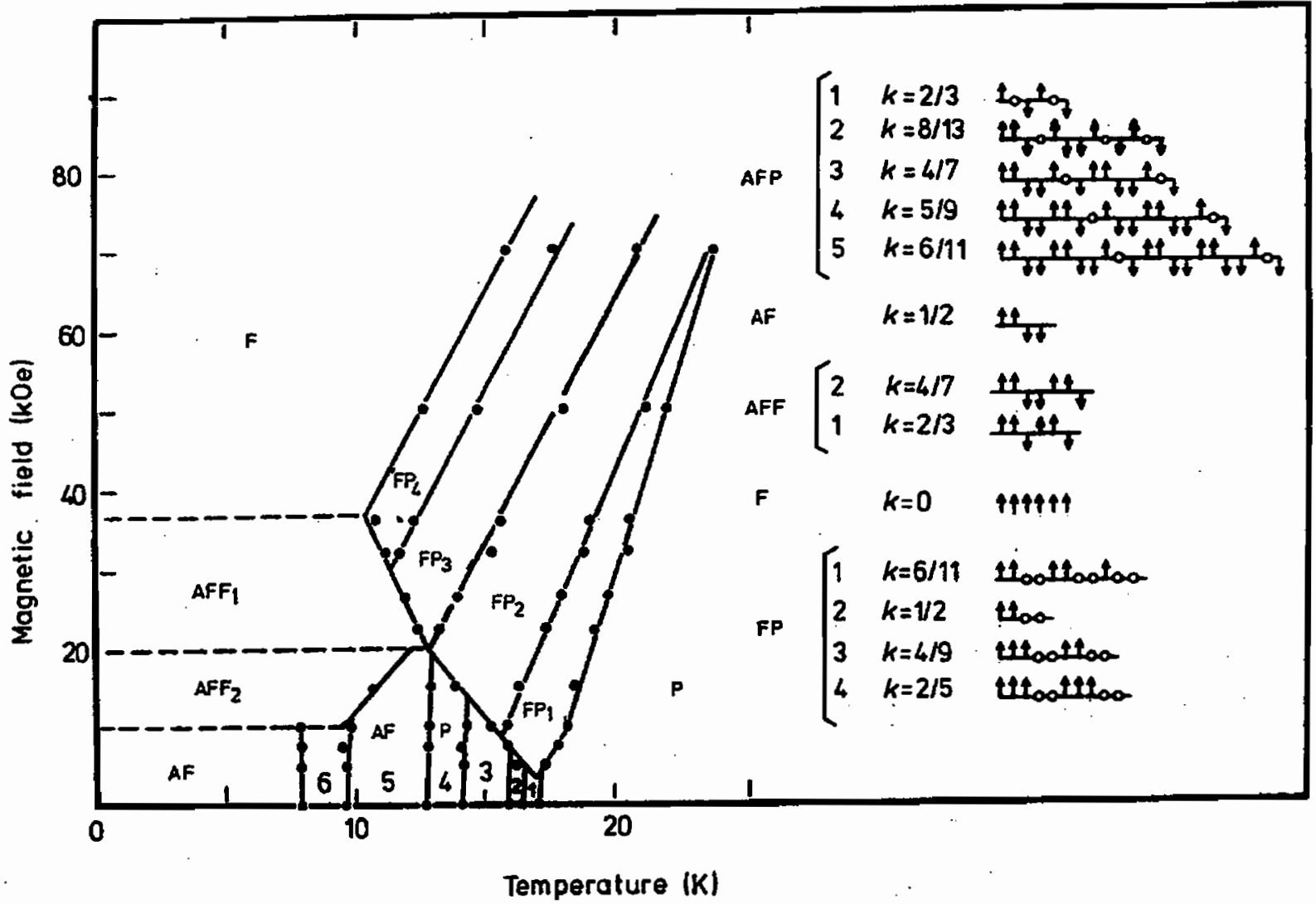

Figura 1.4: Diagrama de fases temperatura-campo magnético do CeSb. Rossat-Mignod, Burlet, Bartholin, Vogt e Langier (1980).

apesar de simples, possui um rico diagrama de fases moduladas, apresentando estruturas comensuráveis e incomensuráveis com a rede subjacente, além do ponto de Lifshitz e pontos de multifases (Selke, 1988; Yeomans, 1988). 


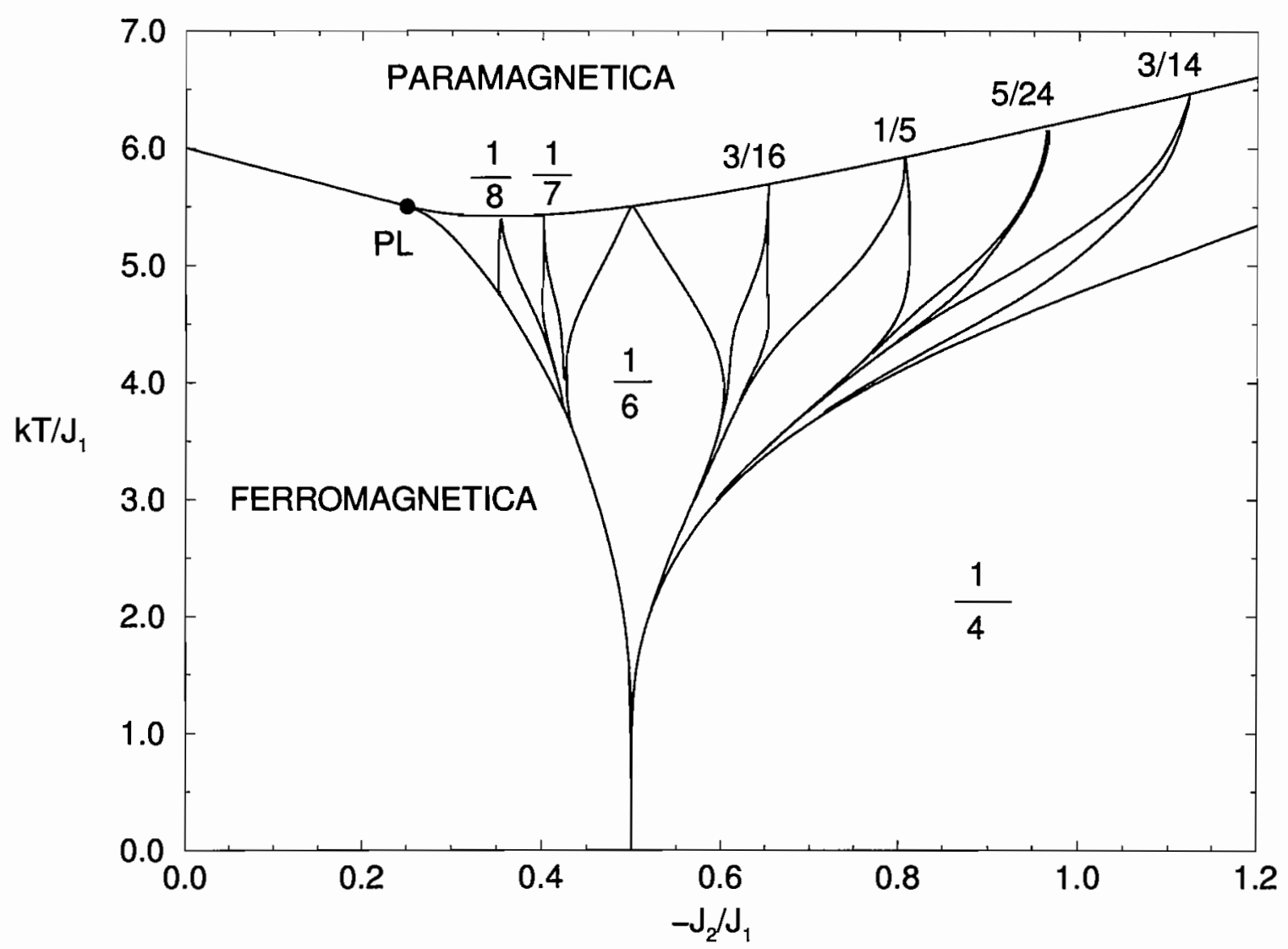

Figura 1.5: Diagrama de fases de campo médio do modelo ANNNI, construído segundo o método de ramificação proposto por Selke e Duxbury (1984). São apresentadas as fases paramagnética, ferromagnética e a região modulada. As frações correspondem ao número de onda das principais fases comensuráveis. O ponto de Lisfshitz PL localiza-se no encontro da linha de segunda ordem, situada entre a fase paramagnética e as fases ordenadas, com a linha de primeira ordem separando a fase ferromagnética da região modulada. 
Pode-se generalizar o modelo ANNNI de várias formas. Por exemplo, pela adição de um campo magnético ou cristalino, pela definição do modelo com variáveis de spin maiores que $1 / 2$, pela adição de interações além de segundos vizinhos, ou pela introdução de desordem. No início da década de oitenta, o estudo do modelo ANNNI com spin 1/2 na presença de um campo magnético uniforme foi realizado por meio de diferentes abordagens. Estudos de campo médio (Yokoi et al., 1981; Ottinger, 1983; Barbosa Filho, 1996), grupo de renormalização no espaço dos momentos (Yokoi, Coutinho-Filho, e Salinas, 1985), expansões em séries de baixas temperaturas (Pokrovsky e Uimin, 1982; Smith e Yeomans, 1982, 1983; Uimin, 1984; Szpilka e Fisher, 1985) mostraram a existência de transições de primeira ordem em baixas temperaturas, o surgimento de linhas de pontos tricríticos no diagrama de fases do modelo, a existência de pontos críticos terminais e de pontos bicríticos. Estudos da adição de interações entre terceiros vizinhos no modelo ANNNI (Selke, Barreto, e Yeomans, 1985; Barreto e Yeomans, 1985) mostraram que a estrutura global dos seus diagramas de fases não sofre alterações qualitativas.

Neste trabalho estamos interessados em investigar a influência de desordem sobre os diagramas de fases do modelo ANNNI. Em particular, estudamos os efeitos da desordem do campo cristalino sobre os diagramas de fases do modelo ANNNI com spin 1 (Cadorin e Yokoi, 1998). O modelo é descrito pela hamiltoniana

$$
\mathcal{H}=-\frac{1}{2} \sum_{\mathbf{r}, \mathbf{r}^{\prime}} J_{\mathbf{r r}} S_{\mathbf{r}} S_{\mathbf{r}^{\prime}}+\sum_{\mathbf{r}} D_{\mathbf{r}} S_{\mathbf{r}}^{2}
$$

onde as somas em $\mathbf{r}$ e $\mathbf{r}^{\prime}$ são sobre todos os sítios da rede e

$$
J_{\mathbf{r r}}=J_{0}\left(\delta_{\mathbf{r}, \mathbf{r}^{\prime}-\hat{\mathbf{x}}}+\delta_{\mathbf{r}, \mathbf{r}^{\prime}+\hat{\mathbf{x}}}+\delta_{\mathbf{r}, \mathbf{r}^{\prime}-\hat{\mathbf{y}}}+\delta_{\mathbf{r}, \mathbf{r}^{\prime}+\hat{\mathbf{y}}}\right)
$$




$$
+J_{1}\left(\delta_{\mathbf{r}, \mathbf{r}^{\prime}-\hat{\mathbf{z}}}+\delta_{\mathbf{r}, \mathbf{r}^{\prime}+\hat{\mathbf{z}}}\right)+J_{2}\left(\delta_{\mathbf{r}, \mathbf{r}^{\prime}-2 \hat{\mathbf{z}}}+\delta_{\mathbf{r}, \mathbf{r}^{\prime}+2 \hat{\mathbf{z}}}\right)
$$

O termo de campo cristalino aleatório $D_{\mathbf{r}}$ obedece a uma distribuição de probabilidades $P\left(D_{\mathbf{r}}\right)$, independente e identicamente distribuída em cada sítio da rede, com valor médio $D_{0}$ e desvio padrão $\sigma$. O caso sem desordem $(\sigma=0)$ corresponde a um campo cristalino constante, ou seja, $D_{\mathbf{r}}=D$ para todo $\mathbf{r}$ na hamiltoniana (1.2) (Cadorin e Yokoi, 1998). Para $\sigma=0$ e $J_{2}=0$, o modelo reduz-se ao modelo de Blume-Capel (Blume, 1966; Capel, 1966).

O planejamento desta tese é como segue: no capítulo 2 realizamos simulações de Monte Carlo do modelo sem desordem $(\sigma=0)$. Os resultados das simulações estão em concordância com estudos de campo médio anteriores (Cadorin e Yokoi, 1998), fornecendo maior credibilidade à aproximação de campo médio. Tendo em vista tal concordância, empregamos a aproximação de campo médio para estudar o caso $\sigma>0$ com mais confiança. Para uma distribuição de probabilidades genérica do campo cristalino, no capítulo 3 obtivemos as equações de campo médio e o funcional da energia livre do modelo. Efetuamos a expansão de Landau do funcional da energia livre e encontramos expressões para as linhas críticas e pontos tricríticos. Nos capítulos 4 e 5 obtivemos os diagramas de fases de campo médio do modelo para as distribuições do campo cristalino delta-bimodal e gaussiana, respectivamente. Finalmente, no capítulo 6 apresentamos um sumário dos resultados obtidos neste trabalho. 


\section{Capítulo 2}

\section{Caso sem desordem: Simulações de Monte Carlo}

\subsection{Introdução}

No contexto de simulações numéricas, o termo "Monte Carlo" foi usado pela primeira vez em um trabalho onde foram desenvolvidas técnicas estatísticas para estudar equações íntegro-diferenciais (Metropolis e Ulam, 1949). A técnica (dinâmica) de Monte Carlo conhecida atualmente como Algoritmo de Metropolis, foi descrita pela primeira vez em 1953 (Metropolis, Rosenbluth, Rosenbluth, Teller, e Teller, 1953). O algoritmo de Metropolis foi o primeiro exemplo de um método de amostragem por importância (importance sampling method), sendo até os dias de hoje o método dessa categoria mais amplamente empregado (Newman e Barkema, 1999).

Simulações de Monte Carlo do modelo ANNNI foram realizadas para estimar os expoentes críticos no ponto de Lifshitz (Selke, 1978), precedendo o primeiro estudo de uma fase espacialmente modulada por meio de simulações (Selke e Fisher, 1979). Simulações de Monte Carlo do modelo ANNNI também foram realizadas para estudar o comportamento tipo "escada do diabo" (devil's 
staircase) (Bak, 1982) do número de onda das fases moduladas como função da temperatura (Rasmussen e Jessen, 1981). Em duas dimensões, simulações do modelo foram realizadas recentemente (Sato e Matsubara, 1999). O método de Monte Carlo também foi empregado em algumas generalizações do modelo ANNNI. Por exemplo, foram realizadas simulações do modelo no caso de diluição de sítios (impurezas) para estudar os efeitos desse tipo de desordem sobre as fases moduladas (Kawasaki, 1984). Outros estudos de Monte Carlo do modelo ANNNI em duas e três dimensões são discutidos nos trabalhos de revisão de Selke e Yeomans (Selke, 1988; Yeomans, 1988).

Outros modelos apresentando fases espacialmente moduladas em seus iagramas de fases também foram estudados por meio de simulações de Monte Carlo. Entre outros, podemos citar a aplicação do método de Monte Carlo para o estudo do modelo de relógio quiral (chiral clock model) de 4 e 6 estados em três dimensões (Scholten e King, 1996) e o uso de técnicas do Grupo de Renormalização Monte-Carlo para o estudo do modelo de relógio quiral de três estados (Houlrik e Jessen, 1986). Estudos de Monte Carlo do modelo DIS (Double Ising Spin) foram recentemente realizados (Neubert, Pleimling, e Siems, 1998).

Embora a maior parte dos trabalhos tenha considerado o modelo ANNNI com spin $S=1 / 2$, algumas generalizações do modelo podem ser importantes para o estudo de propriedades encontradas em sistemas reais. Por exemplo, nos compostos magnéticos CeSb (Rossat-Mignod et al., 1977, 1980) e $\mathrm{PrCo}_{2} \mathrm{Si}_{2}$ (Takeda, Konishi, Deguchi, Iwata, e Shigeoka, 1991), os efeitos do campo cristalino sobre os diagramas de fases são importantes. Nesses compostos foram 
detectadas fases parcialmente desordenadas, ou seja, fases onde existem planos com magnetização nula. Do ponto de vista teórico, estudos do modelo ANNNI com spin $S=1 / 2$ (Nakanishi, 1989; Yokoi, 1991; Cadorin e Yokoi, 1993) indicaram a existência de fases parcialmente desordenadas quando as interações intraplanares são enfraquecidas $\left(J_{0} \ll J_{1}\right)$. Porém, uma aproximação de campo médio mais sofisticada (Nakanishi, 1992) e simulações de Monte Carlo (Rothaus e Selke, 1993) indicaram que as fases parcialmente desordenadas encontradas nos diagramas de fases do modelo ANNNI com spin $S=1 / 2$ são apenas uma deficiência da aproximação de campo médio no caso $J_{0} \ll J_{1}$. Sendo assim, o estudo do modelo ANNNI com spin $S>1 / 2$ pode ser apropriado para o entendimento das fases parcialmente desordenadas observadas nos compostos $\mathrm{CeSb}$ e $\mathrm{PrCo}_{2} \mathrm{Si}_{2}$. Estudos do modelo ANNNI com spin $1 \mathrm{e}$ campo cristalino constante em $T=0$ mostraram que a fase comensurável com período $6,\langle 0++0--\rangle$, na qual dois planos $x y$ com spins $+\mathrm{e}$ dois planos com spins - são intercalados por planos com spins 0, é estável numa região do diagrama de fases, permanecendo estável mesmo em $T>0$ (Cadorin e Yokoi, 1998). Esse resultado não é surpreendente uma vez que, de acordo com a hamiltoniana (1.2), um campo cristalino positivo $(D>0)$ favorece o estado de spin $S=0$. Estudos do modelo na presença de um campo magnético em $T=0$ mostraram a existência de outras fases parcialmente desordenadas (Muraoka, Oda, e Idogaki, 1999).

Embora os resultados obtidos para o modelo ANNNI com spin 1 e campo cristalino constante (Cadorin e Yokoi, 1998) sejam para $J_{0}=J_{1}$, as fases parcialmente desordenadas encontradas nos diagramas de fases do modelo podem 
estar sujeitas a não existirem devido à influência das flutuações térmicas, não levadas em conta na aproximação de campo médio. Portanto, neste capítulo estamos interessados na aplicação do método de Monte Carlo no estudo do modelo ANNNI com spin 1 e campo cristalino constante, definido pela hamiltoniana (1.2) com $D_{\mathbf{r}}=D$ para todo $\mathbf{r}$, com o objetivo de mostrar que a flutuação térmica não desestabiliza a fase $\langle 0++0--\rangle$. Para isso, a partir das simulações esperamos mostrar que a configuração com planos desordenados da fase comensurável de período 6 permanece estável em $T>0$, até uma temperatura na qual ocorre uma transição interna para uma configuração na qual os planos desordenados tornam-se ausentes. De fato, veremos ao longo deste capítulo que as simulações de Monte Carlo do modelo apresentaram evidências desse comportamento, corroborando os resultados de campo médio obtidos anteriormente (Cadorin e Yokoi, 1998).

O estudo de Monte Carlo realizado neste capítulo é de fundamental importância nesta tese, uma vez que os resultados das simulações sustentam o comportamento qualitativo dos diagramas de fases descrito pela aproximação de campo médio.

\subsection{Resultados das simulações}

As simulações foram realizadas em redes cúbicas simples de tamanho $N_{x} \times$ $N_{y} \times N_{z}$ ao longo das direções $x, y$ e $z$, respectivamente, com condições de contorno periódicas em todas as direções. Dizemos que foi efetuado um passo de Monte Carlo por sítio (PMCS) quando todos os sítios da rede forem visitados para a atualização dos seus estados de spin, realizada segundo o algoritmo de 
Metropolis. Neste capítulo, cada fase modulada foi rotulada pelo seu respectivo número de onda, obtido pelas equações (2.2) e (2.3) e correspondente às frações no diagrama de fases de campo médio apresentado na figura 2.1. As letras junto aos números de onda indicam a ausência (letra A) ou a presença (letra B) de planos desordenados na configuração da fase. Os perfis das fases $\frac{1}{4} \mathrm{~A}, \frac{1}{6} \mathrm{~A}$ e $\frac{1}{6} \mathrm{~B}$ são ilustrados na figura 2.1. Os parâmetros do modelo foram fixos em $J_{0}=J_{1},-J_{2} / J_{1}=0.5$ e $D / J_{1}=2.05$. Para o valor do parâmetro de competição $-J_{2} / J_{1}=0.5$, a partir do diagrama de fases de campo médio da figura 2.1 esperamos encontrar apenas a fase 1/6. Por isso, escolhemos para $N_{z}$ um múltiplo de 6. Escolhemos o valor $D / J_{1}=2.05$ por tentativa e erro, de modo a obtermos a melhor evidência das fases $\frac{1}{6} \mathrm{~A}$ e $\frac{1}{6} \mathrm{~B}$. Em todas as simulações descartamos $10^{4}$ PMCS, não encontrando nenhuma dependência com as condições iniciais após esse intervalo de tempo. 


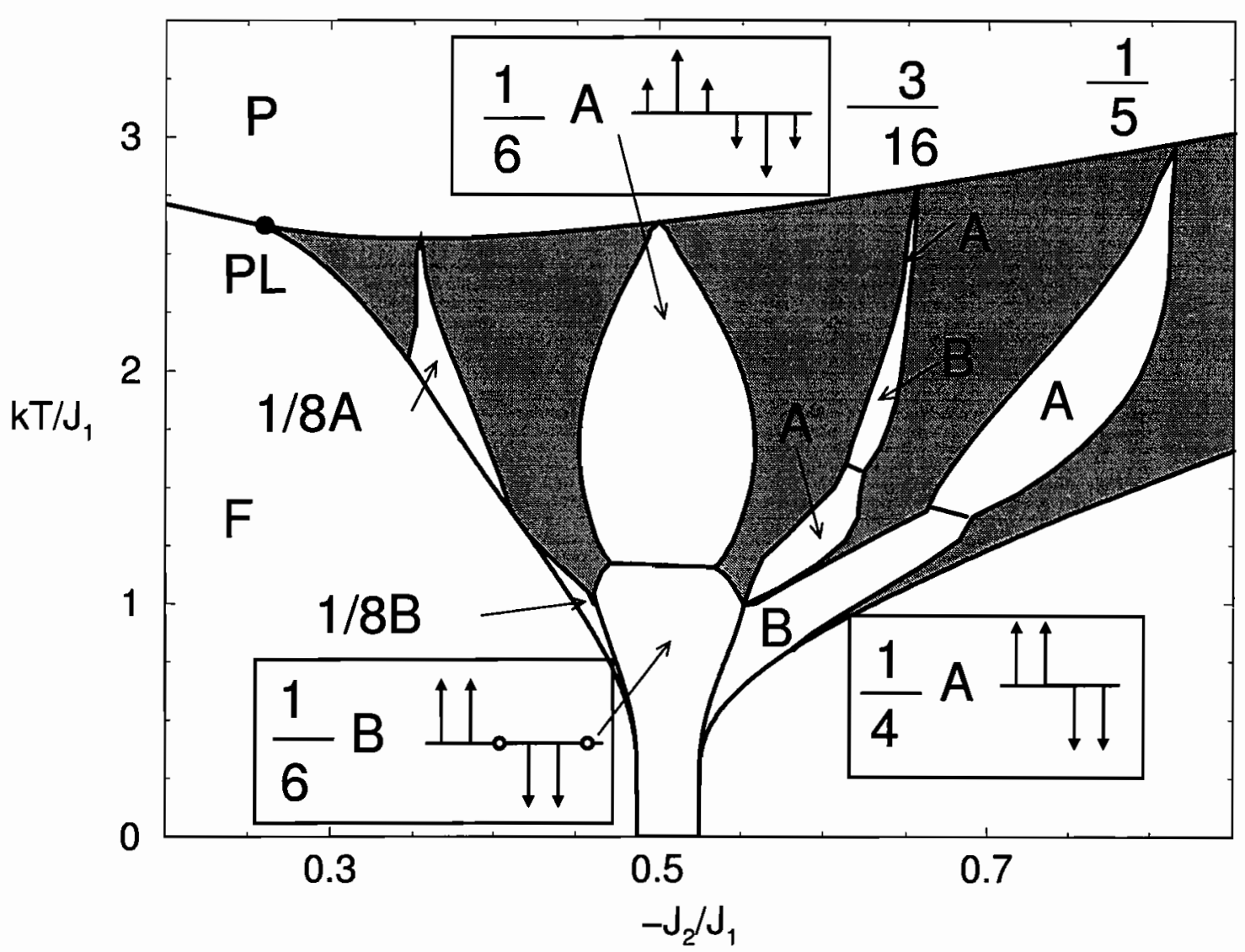

Figura 2.1: Diagrama de fases de campo médio do modelo ANNNI com spin 1 e campo cristalino $D / J_{1}=2.05$, construído para $J_{0}=J_{1}$. São mostradas as fases paramagnética (P), ferromagnética $(\mathrm{F})$ e algumas fases comensuráveis rotuladas pelos seus números de onda e tipos de simetria $\mathrm{A}$ ou $\mathrm{B}$, correspodentes à ausência ou à presença, respectivamente, de planos com magnetização nula. A linha de segunda ordem, separando a fase paramagnética (P) da região ordenada, encontra a linha de primeira ordem, entre a fase ferromagnética (F) e a região modulada, no ponto de Lifshitz (PL). Nas regiões sombreadas existem fases comensuráveis de períodos longos e fases incomensuráveis. 
Na figura 2.2 mostramos valores instantâneos ("fotos") da magnetização por camada

$$
M_{z}=\frac{1}{N_{x} N_{y}} \sum_{x=1}^{N_{x}} \sum_{y=1}^{N_{y}} S_{x, y, z},
$$

obtidos das simulações de um sistema de tamanho $20 \times 20 \times 48$. Dependendo da temperatura observamos diferentes perfis da magnetização. A figura 2.2a mostra um exemplo de perfil da magnetização obtido em uma temperatura baixa, $k T / J_{1}=0.5$. Levando em conta as flutuações, podemos identificar o perfil da fase parcialmente desordenada $\frac{1}{6} \mathrm{~B}$. Em uma temperatura intermediária, $k T / J_{1}=0.675$, o perfil exibe uma grande flutuação de foto para foto, tornando impossivel identificar uma fase definida na figura 2.2b. Entretanto, em uma temperatura ainda mais alta, $k T / J_{1}=1$, a flutuação torna-se pequena novamente, sendo possível identificar a fase $\frac{1}{6} \mathrm{~A}$ no perfil da magnetização da figura $2.2 \mathrm{c}$.

Para estudar com mais detalhes as fases $\frac{1}{6} \mathrm{~A}$ e $\frac{1}{6} \mathrm{~B}$ encontradas nas fotos do perfil da magnetização, fizemos a análise de Fourier de $M_{z}$ (Selke e Fisher, 1979; Rasmussen e Jessen, 1981). Uma vez que a rede é periódica na direção $z$ com período $N_{z}$ par, a magnetização por camada (2.1) pode ser expandida em uma série de Fourier

$$
M_{z}=\sum_{q} M_{q} e^{2 \pi i q z}
$$

onde a soma é efetuada sobre os números de onda

$$
q=\frac{k}{N_{z}}, \quad k=0, \pm 1, \pm 2, \ldots, \pm\left(N_{z}-2\right) / 2, N_{z} / 2
$$

Os coeficientes de Fourier são obtidos da transformada inversa

$$
M_{q}=\frac{1}{N_{z}} \sum_{z=1}^{N_{z}} M_{z} e^{-2 \pi i q z}=\left|M_{q}\right| e^{2 \pi i q \phi_{q}}
$$



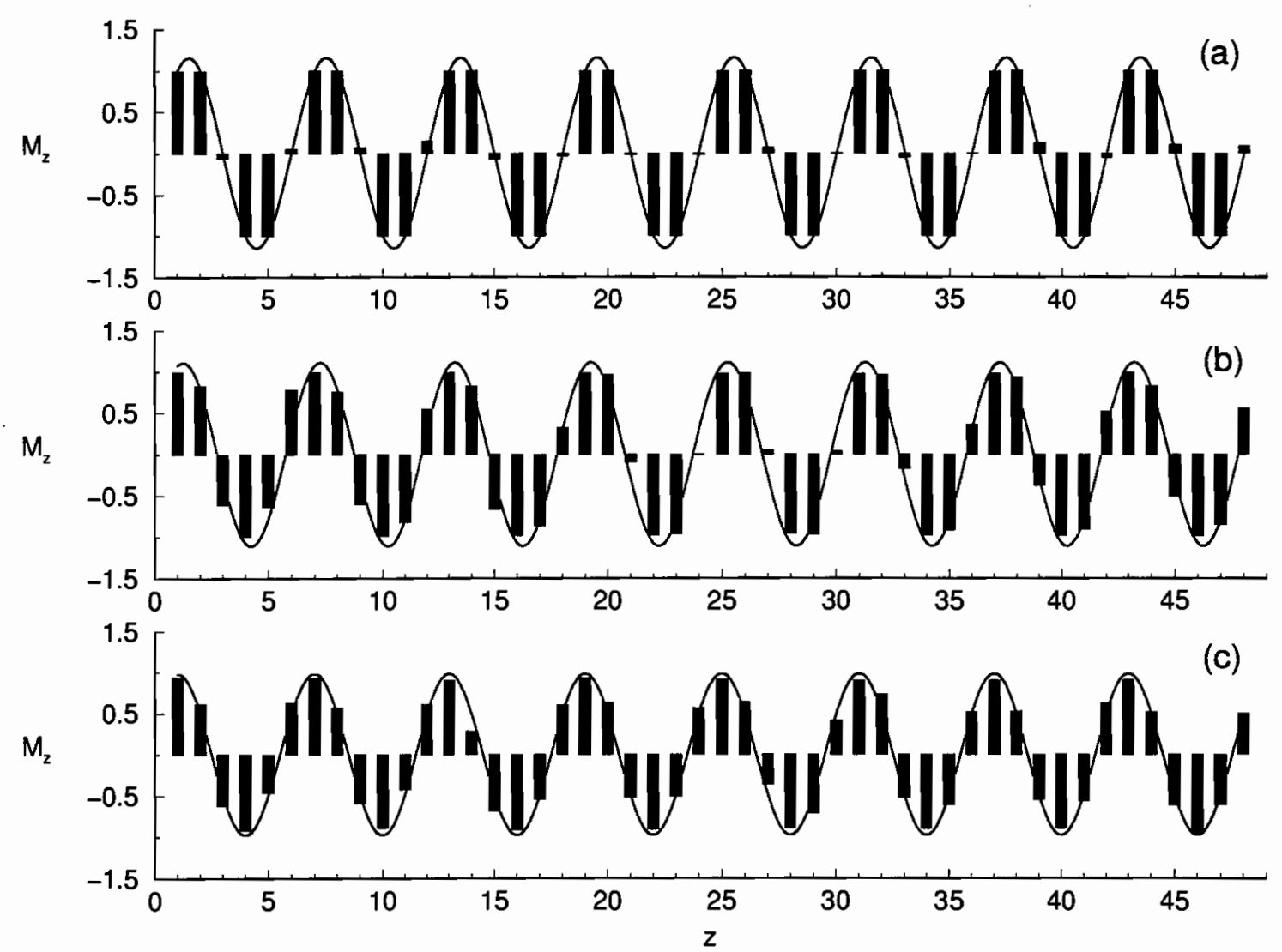

Figura 2.2: Perfis instantâneos da magnetização para um sistema de tamanho $20 \times 20 \times 48$ em (a) $k T / J_{1}=0.5$, (b) $k T / J_{1}=0.675$ e (c) $k T / J_{1}=1$. As linhas contínuas representam o harmônico principal correspondente ao perfil.

onde definimos a amplitude $\left|M_{q}\right|$ e o ângulo de fase $\phi_{q}$ correpondentes ao número de onda $q$.

Medimos a magnetização por camada a cada $10^{2} \mathrm{PMCS}$, sendo computadas a amplitude de Fourier e o ângulo de fase para cada número de onda (2.3). Apenas o harmônico principal $q=\frac{1}{6}$ contribui de forma significativa na série de Fourier (2.2), seguido pela pequena contribuição do terceiro harmônico $q=\frac{1}{2}$ no caso da fase tipo A. Devido às flutuações, existe uma pequena contribuição dos outros harmônicos, como mostrado na figura 2.3. 

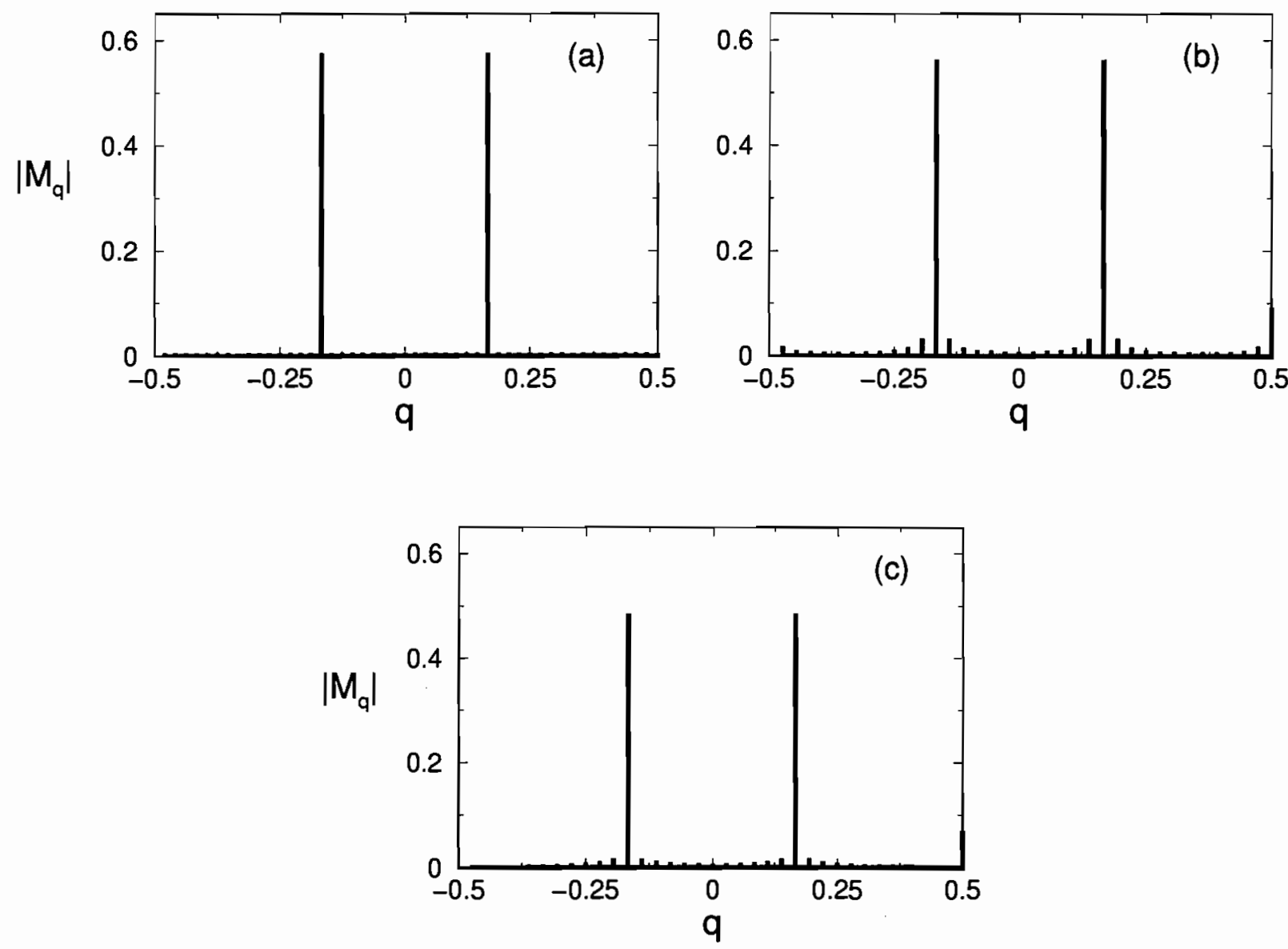

Figura 2.3: Gráficos das amplitudes $\left|M_{q}\right|$ em função dos números de onda $q$ em (a) $k T / J_{1}=$ 0.5 (fase tipo B), (b) $k T / J_{1}=0.675$ (transição entre A e B) e (c) $k T / J_{1}=1$ (fase tipo A). Nos três gráficos observamos claramente a maior contribuição do harmônico principal $q=\frac{1}{6}$. No gráfico (a) observamos que há contribuição apenas do harmônico principal, sendo as pequenas contribuições de todos os outros harmônicos devidas às flutuações. Nos gráficos (b) e (c) notamos a contribuição do terceiro harmônico $q=\frac{1}{2}$. 
Mantendo apenas o harmônico principal $q=\frac{1}{6}$, o perfil da magnetização será dado aproximadamente por

$$
M_{z}=2\left|M_{1 / 6}\right| \cos \left[\frac{\pi}{3}\left(z+\phi_{1 / 6}\right)\right] \text {. }
$$

Essa função senoidal está representada na figura 2.2 pelas linhas contínuas, as quais se ajustam satisfatoriamente aos perfis da magnetização.

$\mathrm{O}$ ângulo de fase $\phi_{1 / 6}$ é de especial interesse porque está relacionado à simetria da fase $\frac{1}{6}$. Um valor inteiro do ângulo $\phi_{1 / 6}$ corresponde à fase $\frac{1}{6} \mathrm{~A}$, um valor semi-inteiro corresponde à fase $\frac{1}{6} \mathrm{~B}$ com planos desordenados e qualquer outro valor de $\phi_{1 / 6}$ corresponde a uma fase sem simetria (Yokoi, 1991). Na figura 2.4 mostramos a evolução do ângulo $\phi_{1 / 6}$ ao longo do tempo (medido em PMCS) para um sistema de tamanho $20 \times 20 \times 48$. Na figura 2.4 a, em uma temperatura baixa, $k T / J_{1}=0.5$, observamos uma pequena flutuação do ângulo de fase em torno do valor $1 / 2$, indicando a estabilidade da fase $\frac{1}{6} \mathrm{~B}$. Na figura $2.4 \mathrm{~b}$, obtida para a temperatura intermediária $k T / J_{1}=0.675$, observamos uma flutuação muito grande do ângulo, indicando que nenhuma das fases $\frac{1}{6} \mathrm{~A}$ e $\frac{1}{6} \mathrm{~B}$ é estável nessa temperatura. Além disso, no intervalo de tempo considerado o valor desse ângulo tende a decrescer, sugerindo um arrasto lento do perfil da fase. Entretanto, em uma temperatura ainda mais alta, $k T / J_{1}=1$, mostramos na figura $2.4 \mathrm{c}$ que a flutuação do ângulo $\phi_{1 / 6}$ torna-se pequena novamente e ocorre em torno do valor 1 , indicando que a fase $\frac{1}{6} \mathrm{~A}$ tornou-se estável.

Para estudar a dependência do ângulo de fase com a temperatura e o tamanho da rede, estimamos a sua distribuição de probabilidades usando os dados coletados das simulações, com rodadas de $1.2 \times 10^{5}$ PMCS e medidas efetuadas 

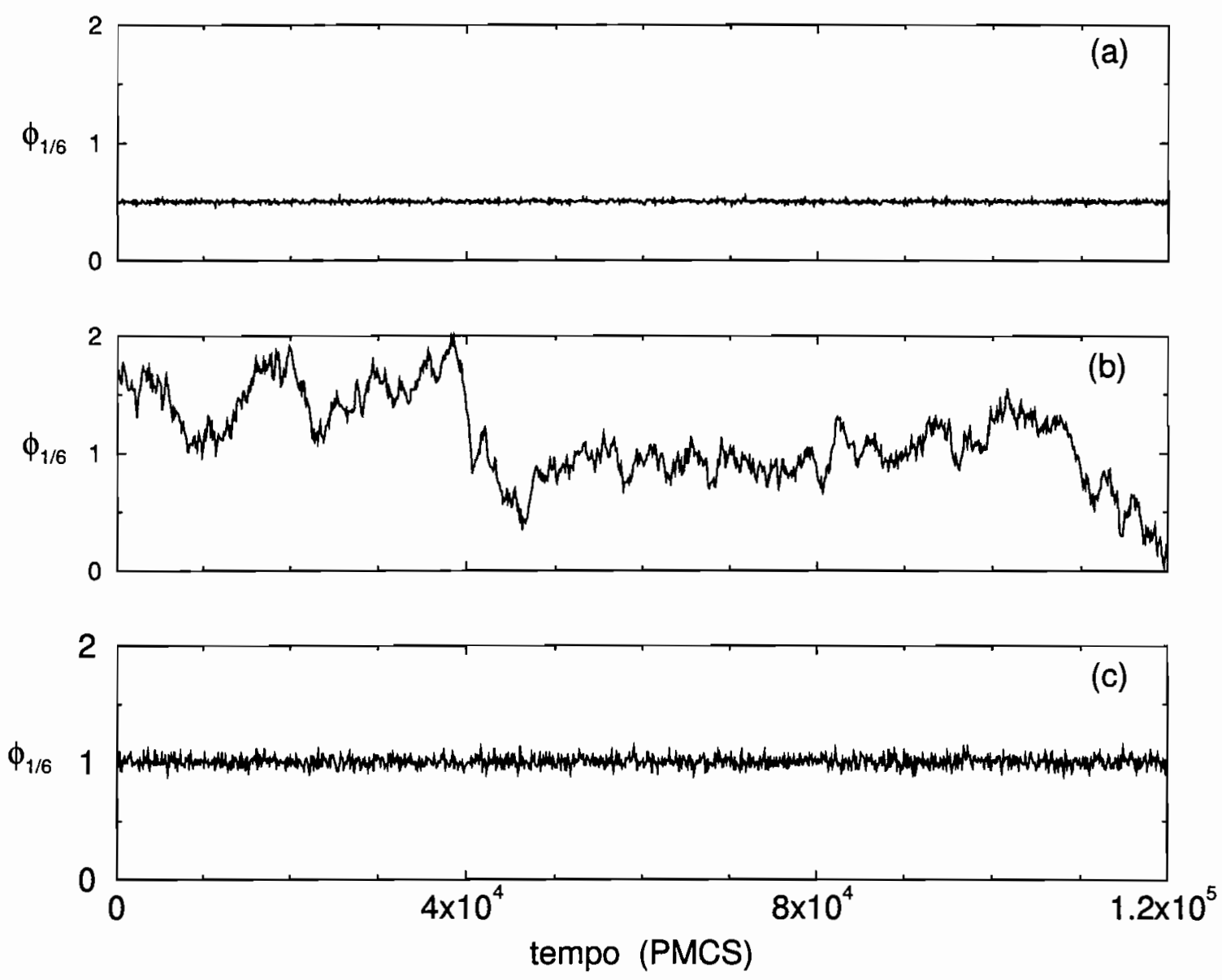

Figura 2.4: Evolução do ângulo de fase $\phi_{1 / 6}$ como função do tempo medido em PMCS em uma rede de tamanho $20 \times 20 \times 48$ para (a) $k T / J_{1}=0.5$, (b) $k T / J_{1}=0.675$ e (c) $k T / J_{1}=1$. Após descartarmos os $10^{4}$ PMCS iniciais, as medidas foram efetuadas a cada $10^{2}$ PMCS.

a cada $10^{2}$ PMCS. Os resultados são mostrados como histogramas normalizados com área unitária. Consideramos primeiro o comportamento do ângulo $\phi_{1 / 6}$ na fase $\frac{1}{6} \mathrm{~A}$. Nas figuras $2.5 \mathrm{a}$ e $2.5 \mathrm{~b}$, os histogramas rotulados (1) correspondem a um sistema de tamanho $20 \times 20 \times 48 \mathrm{em} k T / J_{1}=1$. Os histogramas estão centrados em $\phi_{1 / 6}=0$, em consistência com a fase $\frac{1}{6} \mathrm{~A}$. Para comparação, na figura 2.5a mostramos o histograma (2) correpondente a um sistema menor $10 \times 10 \times 48$ na mesma temperatura de $(1)$, e na figura $2.5 \mathrm{~b}$ o histograma 
(3) correspondente a uma temperatura mais alta, $k T / J_{1}=1.2$ com o mesmo tamanho de rede de (1). Tanto em (2) quanto em (3) observamos o alargamento dos histogramas, indicando flutuações mais fortes para sistemas menores e temperaturas mais altas. Resultados análogos foram obtidos para o ângulo $\phi_{1 / 6}$ referente à fase $\frac{1}{6} \mathrm{~B}$. Nas figuras $2.5 \mathrm{c}$ e $2.5 \mathrm{~d}$, os histogramas rotulados (4) correspondem a um sistema de tamanho $20 \times 20 \times 48 \mathrm{em} k T / J_{1}=0.5$. Os histogramas estão centrados em torno de $\phi_{1 / 6}=0.5$, de acordo com o esperado para a fase $\frac{1}{6} \mathrm{~B}$. Para comparação, na figura $2.5 \mathrm{c}$ mostramos o histograma (5) correspondente a um sistema menor $10 \times 10 \times 48$ na mesma temperatura de (4), e na figura $2.5 \mathrm{~d}$ o histograma (6) correspondente a uma temperatura mais baixa, $k T / J_{1}=0.4$, mas com o mesmo tamanho de rede de (4). Novamente, observamos claramente o alargamento dos histogramas devido a menores tamanhos de rede ou temperaturas mais altas. 

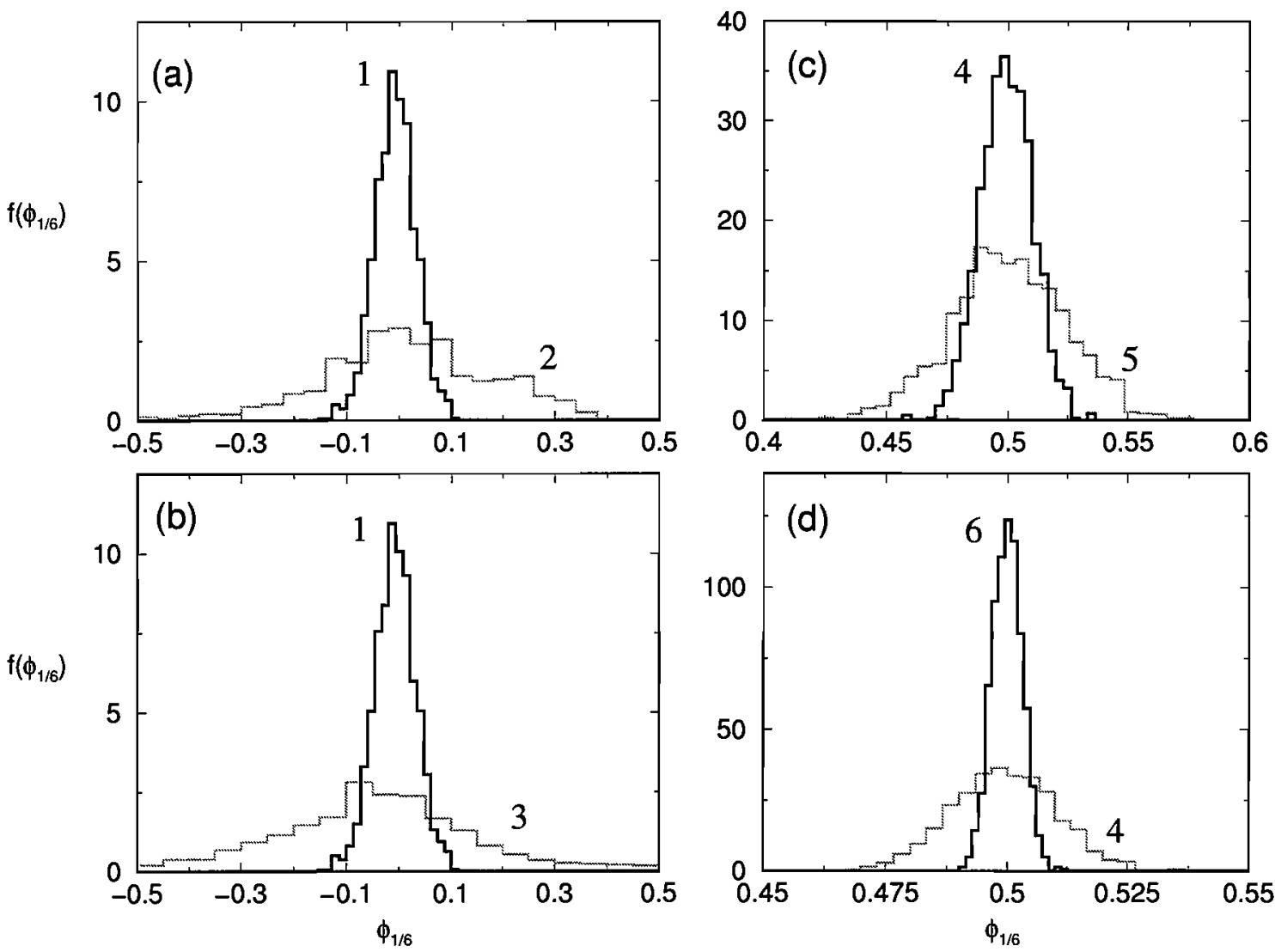

Figura 2.5: Distribuição do ângulo de fase $\phi_{1 / 6}$ do harmônico principal para diferentes temperaturas e tamanhos de rede. Cada histograma representa dados coletados da execução de $1.2 \times 10^{5}$ PMCS, com medidas feitas a cada $10^{2}$ PMCS. Os histogramas são normalizados com área unitária. Os tamanhos dos sistemas e as temperaturas consideradas nas simulações são (1) $20 \times 20 \times 48 \mathrm{em} k T / J_{1}=1$, (2) $10 \times 10 \times 48 \mathrm{em} k T / J_{1}=1$, (3) $20 \times 20 \times 48 \mathrm{em}$ $k T / J_{1}=1.2$, (4) $20 \times 20 \times 48 \mathrm{em} k T / J_{1}=0.5$, (5) $10 \times 10 \times 48 \mathrm{em} k T / J_{1}=0.5 \mathrm{e} \mathrm{(6)}$ $20 \times 20 \times 48 \mathrm{em} k T / J_{1}=0.4$. Os gráficos (a) e (c) mostram maiores flutuações do ângulo de fase devido a tamanhos menores de rede nas fases $\frac{1}{6} \mathrm{~A}$ e $\frac{1}{6} \mathrm{~B}$, respectivamente. Os gráficos (b) and (d) mostram maiores flutuações do ângulo de fase devido a temperaturas mais altas nas fases $\frac{1}{6} \mathrm{~A}$ e $\frac{1}{6} \mathrm{~B}$, respectivamente. 
Também estudamos a dependência da amplitude do harmônico principal $\left|M_{1 / 6}\right|$ com a temperatura e com o tamanho da rede. Nas figuras $2.6 \mathrm{a}$ e $2.6 \mathrm{~b}$, os histogramas rotulados (1) correspondem a um sistema de tamanho $20 \times 20 \times 48 \mathrm{em} k T / J_{1}=1$. Note pela escala do eixo horizontal que a flutuação da amplitude é muito menor que a flutuação do ângulo de fase correspondente. Para comparação, na figura 2.6a mostramos o histograma (2) correspondente a um sistema menor $10 \times 10 \times 48$ na mesma temperatura de (1), e na figura $2.6 \mathrm{~b}$ o histograma (3) correspondente a uma temperatura mais alta $k T / J_{1}=1.2$ com o mesmo tamanho de rede de (1). Ambos os histogramas (2) e (3) são mais largos que (1), novamente indicando maiores flutuações com tamanhos de rede menores ou temperaturas mais altas. Além disso, observamos que o pico do histograma (3) encontra-se em um valor menor da amplitude que o pico de (1), o que ilustra o decrescimento da amplitude em temperaturas mais altas. Resultados análogos foram obtidos no que se refere ao comportamento da amplitude $\left|M_{1 / 6}\right|$ na fase $\frac{1}{6} \mathrm{~B}$. Nas figuras 2.6c e $2.6 \mathrm{~d}$, os histogramas rotulados (4) correspondem a um sistema de tamanho $20 \times 20 \times 48 \mathrm{em} k T / J_{1}=0.5$. Para comparação, na figura 2.6c mostramos o histograma (5) correspondente a um sistema menor $10 \times 10 \times 48$ na mesma temperatura de (4), e na figura $2.6 \mathrm{~d} o$ histograma (6) correspondendo a uma temperatura mais baixa, $k T / J_{1}=0.4$, mas com o mesmo tamanho de rede de (4). Novamente observamos claramente o alargamento dos histogramas para tamanhos de rede menores e temperaturas mais altas, assim como menores amplitudes em temperaturas mais altas. 

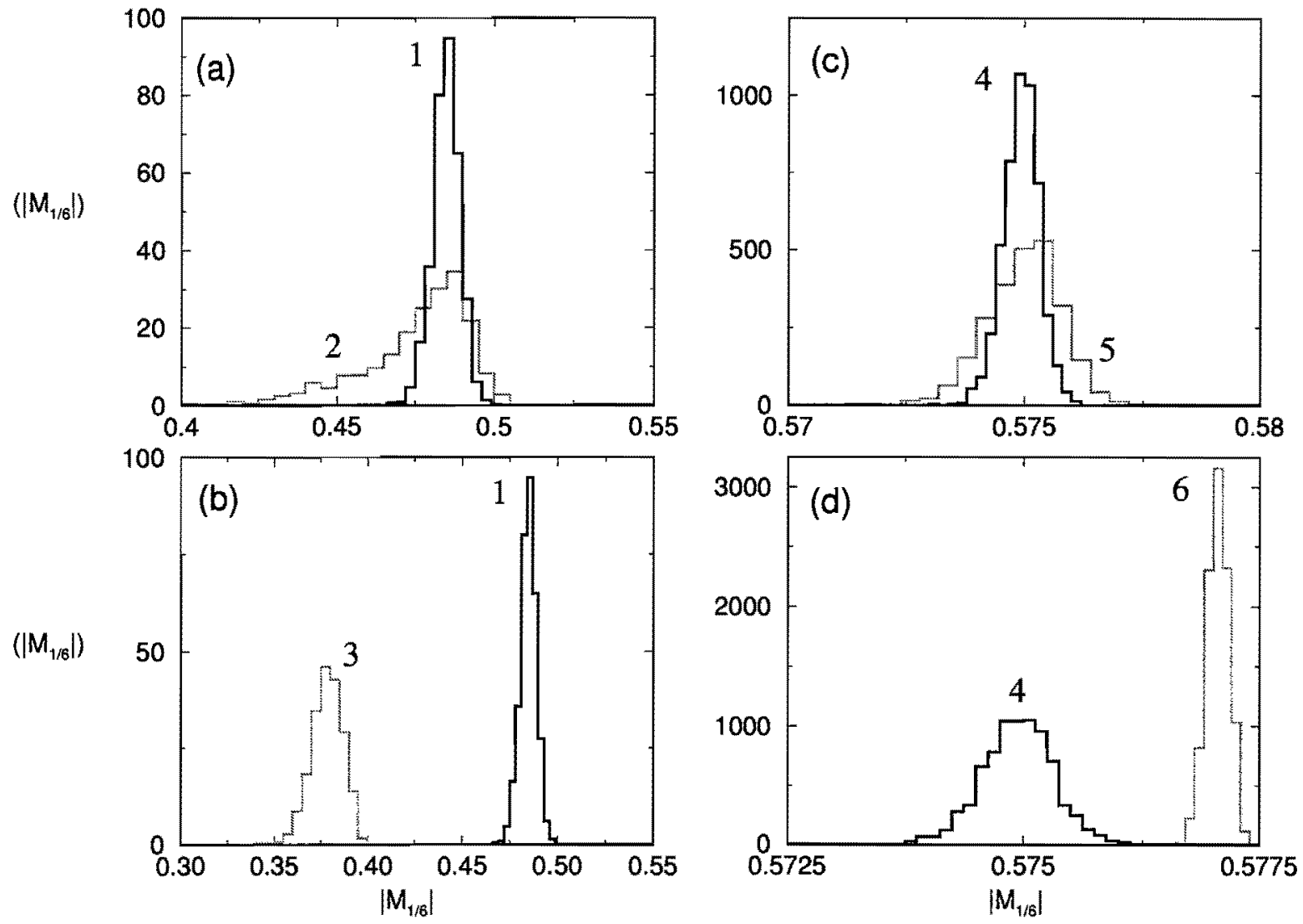

Figura 2.6: Distribuição da amplitude do harmônico principal $\left|M_{1 / 6}\right|$ para diferentes temperaturas e tamanhos de rede. Cada histograma representa dados coletados da execução de $1.2 \times 10^{5}$ PMCS, com medidas feitas a cada $10^{2}$ PMCS. Os histogramas são normalizados com área unitária. Os tamanhos dos sistemas e as temperaturas consideradas nas simulações são (1) $20 \times 20 \times 48 \mathrm{em} k T / J_{1}=1,(2) 10 \times 10 \times 48 \mathrm{em} k T / J_{1}=1$, (3) $20 \times 20 \times 48$

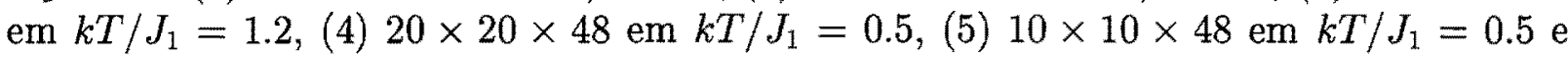
(6) $20 \times 20 \times 48 \mathrm{em} k T / J_{1}=0.4$. Os gráficos (a) e (c) mostram maiores flutuações da amplitude devido a tamanhos menores de rede nas fases $\frac{1}{6} \mathrm{~A}$ e $\frac{1}{6} \mathrm{~B}$, respectivamente. Os gráficos (b) and (d) mostram maiores flutuações da amplitude devido a temperaturas mais altas nas fases $\frac{1}{6} \mathrm{~A}$ e $\frac{1}{6} \mathrm{~B}$, respectivamente. 
Na figura 2.7 mostramos os histogramas do ângulo de fase $\phi_{1 / 6}$ e da amplitude $\left|M_{1 / 6}\right|$ em uma temperatura intermediária, $k T / J_{1}=0.675$, próximo à temperatura de transição entre as fases $\frac{1}{6} \mathrm{~A}$ e $\frac{1}{6} \mathrm{~B}$. Histogramas (1) e (2) correspondem aos tamanhos de rede $20 \times 20 \times 48$ e $10 \times 10 \times 48$, respectivamente. Histogramas para o ângulo de fase mostrados na figura. 2.7a indicam uma grande flutuação tanto para redes pequenas quanto grandes, em concordância com a evolução temporal mostrada na figura 2.4c. Contrariamente, os histogramas da amplitude mostrados na figura $2.7 \mathrm{~b}$ apresentam picos muito bem definidos, com uma flutuação ligeiramente maior para o sistema menor. Esses resultados são consistentes com um perfil de magnetização senoidal que se desloca quase livremente. 

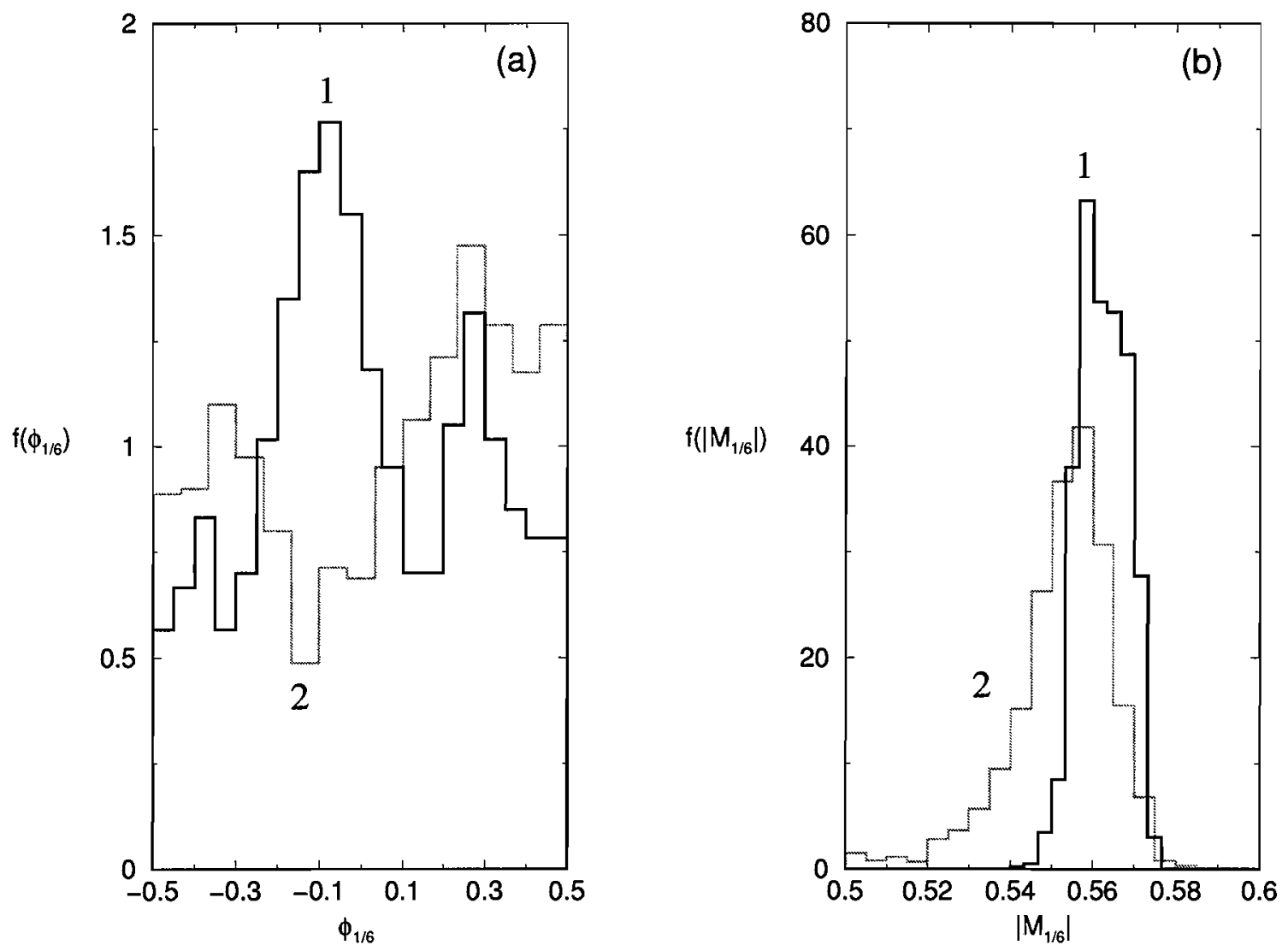

Figura 2.7: Distribuições do ângulo de fase $\phi_{1 / 6}$ e da amplitude $\left|M_{1 / 6}\right|$ do harmônico principal para os tamanhos de rede (1) $20 \times 20 \times 48$ e (2) $10 \times 10 \times 48 \mathrm{em} \mathrm{kT} / J_{1}=0.675$. Cada histograma representa dados coletados da execução de $1.2 \times 10^{5}$ PMCS com medidas feitas a cada $10^{2}$ PMCS. Os histogramas são normalizados com área unitária. O gráfico (a) ilustra a grande flutuação do ângulo de fase observada nessa temperatura tanto para redes grandes quanto para redes menores, embora a flutuação da amplitude seja menor para redes maiores, como mostrado no gráfico (b). 
Para a estimativa da temperatura de transição entre as fases $\frac{1}{6} \mathrm{~A}$ e $\frac{1}{6} \mathrm{~B}$, estudamos o calor específico como função da temperatura considerando um sistema de tamanho $20 \times 20 \times 36$. Realizamos $10^{4} \mathrm{PMCS}$ em cada temperatura, com medidas efetuadas a cada 10 PMCS. O gráfico da figura 2.8 apresenta dois picos. O pico na temperatura mais alta $k T / J_{1} \approx 1.39$ corresponde à transição para a fase desordenada. O pico mais estreito observado na temperatura mais baixa $k T / J_{1} \approx 0.675$ corresponde à transição interna entre as fases $\frac{1}{6} \mathrm{~A}$ e $\frac{1}{6} \mathrm{~B}$. Para comparação citamos os valores de campo médio $k T / J_{1}=2.623 \mathrm{e}$ $k T / J_{1}=1.164$, respectivamente, para essas temperaturas de transição. 


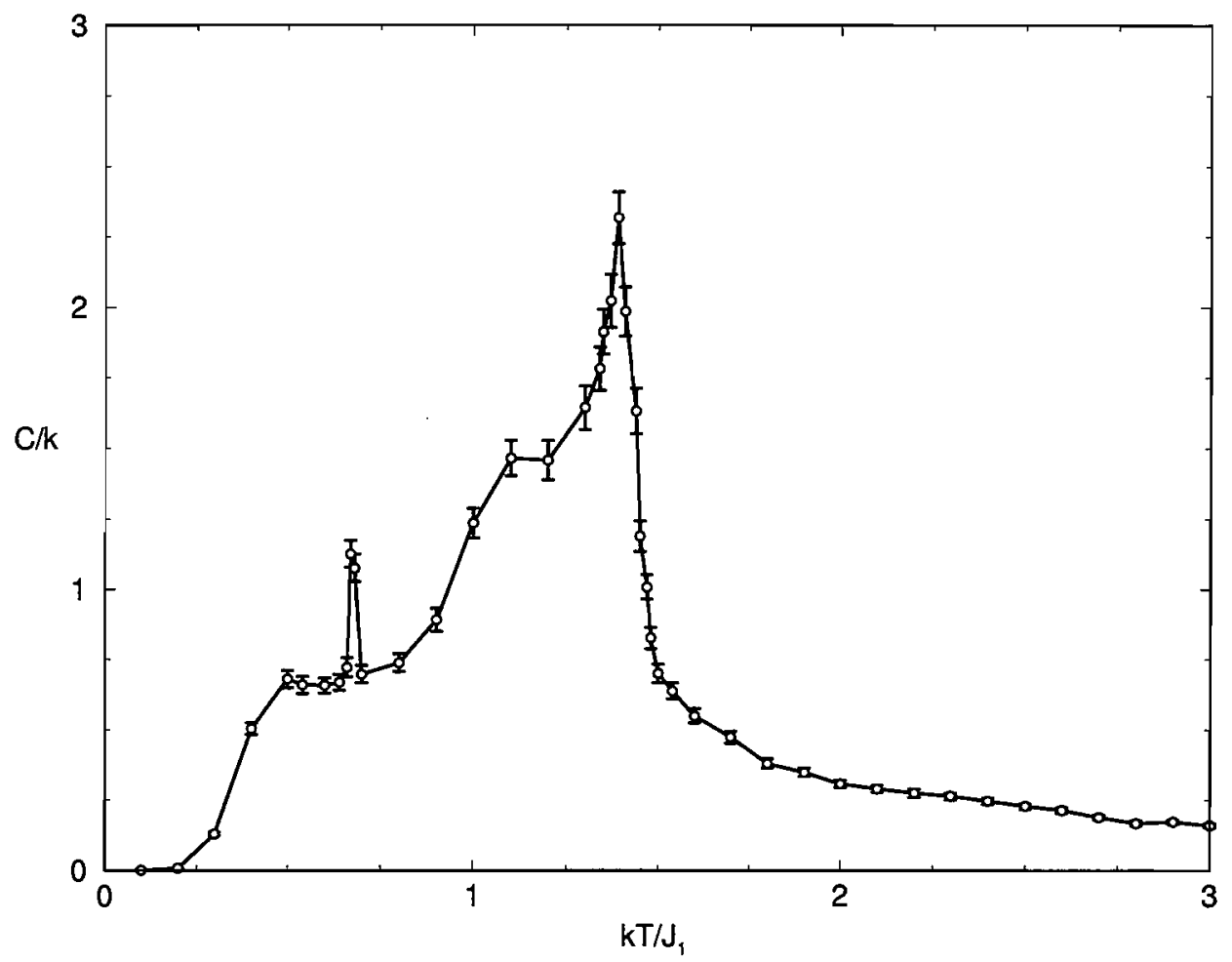

Figura 2.8: Calor específico por spin como função da temperatura para um sistema de tamanho $20 \times 20 \times 36,-J_{2} / J_{1}=0.5$ e $D / J_{1}=2.05$. Executamos $10^{4}$ PMCS em cada temperatura com medidas feitas a cada 10 PMCS. O pico maior em $k T / J_{1} \approx 1.39$ marca a transição para a fase paramagnética, ao passo que o pico menor encontrado na temperatura mais baixa $k T / J_{1} \approx 0.675$ indica a transição interna entre as fases $\frac{1}{6} \mathrm{~A}$ e $\frac{1}{6} \mathrm{~B}$. 


\section{Capítulo 3}

\section{Caso com desordem: Aproximação de campo médio}

\subsection{Introdução}

Conceitualmente, podemos distinguir três tipos de desordem em um sólido. Suponhamos um cristal monoatômico perfeito, no qual todas as arestas possuem o mesmo comprimento e todos os sítios têm o mesmo número de coordenação. A desordem de ligações corresponde à deformação desse cristal de tal forma que os comprimentos das arestas passem a ser desordenadamente diferentes uns dos outros, enquanto o número de coordenação de cada sítio permanece o mesmo. Em sólidos reais essa desordem corresponde, por exemplo, aos deslocamentos dos átomos de um cristal das suas posições de equilíbrio, devido a efeitos térmicos. O segundo tipo de aleatoriedade é a desordem topológica. Nesse caso as deformações alteram não apenas os comprimentos das arestas, mas também o número de coordenação dos sítios da rede. A desordem topológica necessariamente inclui a desordem de ligações e está presente na formação dos materiais vítreos ou amorfos. Finalmente, a desordem quimi$c a$ é aquela na qual os sítios da rede são ocupados aleatoriamente por átomos 
distintos. Por exemplo, a liga binária $\mathrm{A}_{x} \mathrm{~B}_{1-x}$ é um composto apresentando desordem química. No caso em que "A" é um átomo magnético e "B" é um átomo não magnético, a desordem química está relacionada ao fenômeno de percolação em magnetos com diluição de sítios (Stinchcombe, 1983).

A desordem pode ser acrescentada a um sistema puro de diferentes maneiras, como por exemplo, pela diluição de sítios ou ligações, ou pela introdução de interações e campos magnéticos ou cristalinos aleatórios. Entre os sistemas desordenados, um dos mais desafiadores do ponto de vista teórico é o vidro de spin. Os ingredientes básicos de um vidro de spin são a desordem das ligações e a frustração, oriunda da competição entre os ordenamentos ferro e antiferromagnéticos (Moorjani e Coey, 1984; Binder e Young, 1986; Mézard, Parisi, e Virasoro, 1987; Fischer e Hertz, 1993; Mydosh, 1993; Young, 1997).

O diagrama de fases do modelo ANNNI e suas generalizações é o resultado de um equilíbrio sutil entre as diferentes fases moduladas (metaestáveis) possíveis. Esse fato nos conduz à questão de como a adição de desordem ao modelo interfere nesse equilíbrio e quais as suas consequências sobre os diagramas de fases. Considerando impurezas recozidas ("annealed") no modelo ANNNI, estudos de campo médio mostraram que o diagrama de fases sofre pequenas alterações, mas a estrutura geral das fases moduladas se mantém inalterada (Roeder e Yeomans, 1985). O modelo ANNNI com impurezas recozidas pode ser mapeado ao modelo ANNNI com spin 1 e campo cristalino $D<0$ (Cadorin e Yokoi, 1998), onde o parâmetro $D$ está relacionado ao potencial químico controlando o número de impurezas no sistema (Roeder e Yeomans, 1985). No caso da adição de desordem congelada ("quenched"), estudos baseados em argumen- 
tos de paredes de domínios mostraram que as fases comensuráveis de longos períodos são instáveis em três dimensões (Fishman e Yeomans, 1985; Bak, Coppersmith, Shapir, Fishman, e Yeomans, 1985). Além disso, foram realizadas simulações de Monte Carlo em uma versão "quenched" diluída do modelo ANNNI (Kawasaki, 1984), com possível aplicação na interpretação das propriedades do ponto de Lifshitz e suas vizinhanças em amostras de $\mathrm{Mn}_{1-p} \mathrm{~T}_{p} \mathrm{P}$, sendo $\mathrm{T}=\mathrm{Ni}$, Mo, W e Co (Ziěba, Fjellvåg, e Kjekshus, 1988; Ziěba, Becerra, Oliveira Jr., Fjellvåg, e Kjekshus, 1992; Ziěba, Becerra, Fjellvåg, Oliveira Jr., e Kjekshus, 1992). Ainda no caso congelado, o modelo ANNNI com spin $1 / 2$ foi estudado na presença de um campo magnético aleatório (Tamashiro, Yokoi, e Salinas, 1997). Para uma distribuição bimodal simétrica do campo, mostrou-se que campos fracos não alteram qualitativamente os diagramas de fases. Para campos moderados, observou-se a existência de pontos tricríticos e de fases parcialmente desordenadas. Finalmente, no caso de campos intensos, as fases moduladas são destruídas (Tamashiro et al., 1997).

Os efeitos de um campo cristalino aleatório no modelo de Blume-Capel (Blume, 1966; Capel, 1966) foram estudados na aproximação de campo médio para as distribuições de probabilidades do campo cristalino delta-bimodal (Borelli e Carneiro, 1996; Benyoussef, Biaz, Saber, e Touzani, 1987; Carneiro, Henriques, e Salinas, 1989) e gaussiana (Carneiro, Henriques, e Salinas, 1990). Nesses trabalhos verificou-se que a aleatoriedade do campo cristalino exerce influência sobre as transições de primeira ordem do modelo, podendo desestabilizar os pontos tricríticos, dando origem a pontos críticos terminais e pontos bicríticos. 
Em síntese, os trabalhos expostos acima mostram que a desordem pode afetar significativamente os diagramas de fases, exercendo influência sobre a ordem das transições de fases, a estabilidade dos pontos multicríticos e o comportamento das fases moduladas. Com essa motivação, nesta tese investigamos a influência da desordem do campo cristalino sobre os diagramas de fases do modelo ANNNI com spin 1 definido pela hamiltoniana (1.2).

\subsection{Equações de campo médio e funcional da energia livre}

Estamos considerando uma desordem magnética congelada, que é o tipo de desordem encontrada em materiais magnéticos reais (Stinchcombe, 1983). Para esse tipo de desordem, a energia livre $G$ é dada pela média das energias livres $G\left(\left\{D_{\mathbf{r}}\right\}\right)$ sobre as possíveis configurações do campo cristalino $\left\{D_{\mathbf{r}}\right\}$ (Stinchcombe, 1983), ou seja,

$$
G=\left\langle G\left(\left\{D_{\mathbf{r}}\right\}\right)\right\rangle_{c}
$$

onde

$$
\langle(\cdots)\rangle_{c}=\int_{-\infty}^{\infty} \prod_{\mathbf{r}} \mathrm{d} D_{\mathbf{r}}(\cdots) P\left(D_{\mathbf{r}}\right) .
$$

Para a obtenção da energia livre (3.1), utilizamos o teorema variacional de Bogoliubov (Falk, 1970; Callen, 1985)

$$
G\left(\left\{D_{\mathbf{r}}\right\}\right) \leq G_{0}\left(\left\{D_{\mathbf{r}}\right\}\right)+\left\langle\mathcal{H}\left(\left\{D_{\mathbf{r}}\right\}\right)-\mathcal{H}_{0}\left(\left\{D_{\mathbf{r}}\right\}\right)\right\rangle_{0}
$$

onde $\langle\cdots\rangle_{0}$ é a média em relação à hamiltoniana tentativa $\mathcal{H}_{0} \mathrm{e}$

$$
\begin{aligned}
G_{0} & =-\frac{1}{\beta} \ln Z_{0} \\
Z_{0} & =\sum_{\{S\}} e^{-\beta \mathcal{H}_{0}}
\end{aligned}
$$


onde a soma em (3.5) é sobre todas as possíveis configurações dos spins. Portanto, a energia livre (3.1) satisfaz a desigualdade

$$
G \leq\left\langle G_{0}\left(\left\{D_{\mathbf{r}}\right\}\right)+\left\langle\mathcal{H}\left(\left\{D_{\mathbf{r}}\right\}\right)-\mathcal{H}_{0}\left(\left\{D_{\mathbf{r}}\right\}\right)\right\rangle_{0}\right\rangle_{c} \equiv \Phi
$$

Uma vez que estamos desenvolvendo um cálculo variacional, a melhor escolha para $\mathcal{H}_{0}$ é aquela que leva em conta o máximo de informação sobre a hamiltoniana original (1.2), mas ainda permitindo a solução do lado direito da equação (3.6). Portanto, incluímos em $\mathcal{H}_{0}$ o segundo termo no lado direito da hamiltoniana (1.2). O parâmetro variacional foi introduzido como um campo efetivo $\eta_{\mathbf{r}}$ que depende do particular sítio $\mathbf{r}$, isto é,

$$
\mathcal{H}_{0}=-\sum_{\mathbf{r}} \eta_{\mathbf{r}} S_{\mathbf{r}}+\sum_{\mathbf{r}} D_{\mathbf{r}} S_{\mathbf{r}}^{2}
$$

Usando as equações (3.4), (3.5) e (3.7) obtemos

$$
\begin{aligned}
G_{0}+\left\langle\mathcal{H}-\mathcal{H}_{0}\right\rangle_{0}= & -\frac{1}{\beta} \sum_{\mathbf{r}} \ln \left(1+2 e^{-\beta D_{\mathbf{r}}} \cosh \beta \eta_{\mathbf{r}}\right) \\
& -\frac{1}{2} \sum_{\mathbf{r}, \mathbf{r}^{\prime}} J_{\mathbf{r r}^{\prime}}\left\langle S_{\mathbf{r}}\right\rangle_{0}\left\langle S_{\mathbf{r}^{\prime}}\right\rangle_{0}+\sum_{\mathbf{r}} \eta_{\mathbf{r}}\left\langle S_{\mathbf{r}}\right\rangle_{0}
\end{aligned}
$$

onde

$$
\left\langle S_{\mathbf{r}}\right\rangle_{0}=\frac{2 e^{-\beta D_{\mathbf{r}}} \sinh \beta \eta_{\mathbf{r}}}{1+2 e^{-\beta D_{\mathbf{r}}} \cosh \beta \eta_{\mathbf{r}}}
$$

A seguir definimos

$$
m_{\mathbf{r}}=\left\langle\left\langle S_{\mathbf{r}}\right\rangle_{0}\right\rangle_{c}
$$

e usando o fato de que a distribuição de probabilidades $P\left(D_{\mathbf{r}}\right)$ é independente para cada sítio $\mathbf{r}$ e identicamente distribuída, obtemos

$$
\left\langle\left\langle S_{\mathbf{r}}\right\rangle_{0}\left\langle S_{\mathbf{r}^{\prime}}\right\rangle_{0}\right\rangle_{c}=\left\langle\left\langle S_{\mathbf{r}}\right\rangle_{0}\right\rangle_{c}\left\langle\left\langle S_{\mathbf{r}^{\prime}}\right\rangle_{0}\right\rangle_{c}=m_{\mathbf{r}} m_{\mathbf{r}^{\prime}}
$$


A seguir calculamos a média da equação (3.8) sobre as configurações do campo cristalino e usamos a equação (3.11) para obter o funcional da energia livre

$$
\Phi=-\frac{1}{\beta} \sum_{\mathbf{r}}\left\langle\ln \left(1+2 e^{-\beta D_{\mathbf{r}}} \cosh \beta \eta_{\mathbf{r}}\right\rangle_{c}-\frac{1}{2} \sum_{\mathbf{r}, \mathbf{r}^{\prime}} J_{\mathbf{r r}} m_{\mathbf{r}} m_{\mathbf{r}^{\prime}}\right.
$$

onde

$$
m_{\mathbf{r}}=\left\langle\frac{2 e^{-\beta D_{\mathbf{r}}} \sinh \beta \eta_{\mathbf{r}}}{1+2 e^{-\beta D_{\mathbf{r}}} \cosh \beta \eta_{\mathbf{r}}}\right\rangle_{c},
$$

e a integral múltipla na definição (3.2) torna-se a integral simples relacionada ao particular sítio $\mathbf{r}$.

O parâmetro variacional $\eta_{\mathbf{r}}$ é obtido aplicando-se a condição de ponto estacionário para $\Phi$, isto é,

$$
\frac{\partial \Phi}{\partial \eta_{\mathbf{r}}}=0
$$

o que implica

$$
\eta_{\mathbf{r}}=\sum_{\mathbf{r}} J_{\mathbf{r r}^{\prime}} m_{\mathbf{r}^{\prime}}
$$

Substituindo explicitamente a definição (1.3) para $J_{\mathbf{r r}^{\prime}}$ na equação (3.15) obtemos

$$
\eta_{\mathbf{r}}=4 J_{0} m_{\mathbf{r}}+J_{1}\left(m_{\mathbf{r}-\hat{\mathbf{z}}}+m_{\mathbf{r}+\hat{\mathbf{z}}}\right)+J_{2}\left(m_{\mathbf{r}-2 \hat{\mathbf{z}}}+m_{\mathbf{r}+2 \hat{\mathbf{z}}}\right) .
$$

Para modelos do tipo ANNNI, esperamos que a modulação ocorra ao longo do eixo $z$ de iterações competitivas. Uma vez que as interações intracamadas $J_{0}$ são ferromagnéticas, também esperamos um ordenamento ferromagnético nos planos $x y$. Finalmente, sendo a desordem identicamente distribuída, não esperamos que o campo cristalino aleatório modifique o ordenamento magnético. Assim, podemos impor

$$
\eta_{\mathbf{r}} \equiv \eta_{z} \quad(z=\mathbf{r} \cdot \hat{\mathbf{z}})
$$


e consequentemente,

$$
m_{\mathbf{r}} \equiv m_{z}
$$

Portanto, as equações de campo médio (3.13) e (3.16) ficam

$$
\begin{aligned}
m_{z} & =\left\langle\frac{2 e^{-\beta D} \sinh \beta \eta_{z}}{1+2 e^{-\beta D} \cosh \beta \eta_{z}}\right\rangle_{c}, \\
\eta_{z} & =4 J_{0} m_{z}+J_{1}\left(m_{z-1}+m_{z+1}\right)+J_{2}\left(m_{z-2}+m_{z+2}\right),
\end{aligned}
$$

onde

$$
\langle(\cdots)\rangle_{c}=\int_{-\infty}^{\infty} \mathrm{d} D(\cdots) P(D) .
$$

O funcional da energia livre fica

$$
\begin{aligned}
N^{-3} \Phi\left(T, D, N ; \eta_{z}\right)= & -\frac{1}{\beta N} \sum_{z}\left\langle\ln \left(1+2 e^{-\beta D} \cosh \beta \eta_{z}\right)\right\rangle_{c} \\
& -\frac{1}{2 N} \sum_{z}\left[4 J_{0} m_{z}^{2}+J_{1} m_{z}\left(m_{z-1}+m_{z+1}\right)\right. \\
& \left.+J_{2} m_{z}\left(m_{z-2}+m_{z+2}\right)\right]+\frac{1}{N} \sum_{z} \eta_{z} m_{z}
\end{aligned}
$$

onde as somas são efetuadas para $1 \leq z \leq N$. O funcional (3.22) pode ser escrito em uma forma mais conveniente para o desenvolvimento da expansão de Landau e para o estudo do limite $T \rightarrow 0$. Uma vez que

$$
\frac{\partial}{\partial \eta_{z}}\left[\left\langle\ln \left(1+2 e^{-\beta D} \cosh \beta \eta_{z}\right)\right\rangle_{c}\right]=\beta\left\langle\frac{2 e^{-\beta D} \sinh \beta \eta_{z}}{1+2 e^{-\beta D} \cosh \beta \eta_{z}}\right\rangle_{c}=\beta m_{z}
$$

podemos escrever

$$
\left\langle\ln \left(1+2 e^{-\beta D} \cosh \beta \eta_{z}\right)\right\rangle_{c}=\beta \int_{0}^{\eta_{z}} m_{z} \mathrm{~d} \eta_{z}+\left\langle\ln \left(1+2 e^{-\beta D}\right)\right\rangle_{c} .
$$

Integrando por partes,

$$
\int_{0}^{\eta_{z}} m_{z} \mathrm{~d} \eta_{z}=m_{z} \eta_{z}-\int_{0}^{m_{z}} \eta_{z} \mathrm{~d} m_{z}
$$


Portanto,

$$
\begin{aligned}
\left\langle\ln \left(1+2 e^{-\beta D} \cosh \beta \eta_{z}\right)\right\rangle_{c}= & \beta m_{z} \eta_{z}-\beta \int_{0}^{m_{z}} \eta_{z} \mathrm{~d} m_{z} \\
& +\left\langle\ln \left(1+2 e^{-\beta D}\right)\right\rangle_{c}
\end{aligned}
$$

Substituindo a equação (3.26) no funcional (3.22), obtemos

$$
\begin{aligned}
N^{-3} \Phi= & -\frac{1}{\beta}\left\langle\ln \left(1+2 e^{-\beta D}\right)\right\rangle_{c}+\frac{1}{N} \sum_{z} \int_{0}^{m_{z}} \eta_{z} \mathrm{~d} m_{z} \\
& -\frac{1}{2 N} \sum_{z}\left[4 J_{0} m_{z}^{2}+J_{1} m_{z}\left(m_{z-1}+m_{z+1}\right)\right. \\
& \left.+J_{2} m_{z}\left(m_{z-2}+m_{z+2}\right)\right] .
\end{aligned}
$$

Da equação (3.6), a melhor estimativa para a energia livre $G$ é obtida da minimização do funcional $\Phi$ em relação aos parâmetros variacionais $\eta_{z}$, ou seja,

$$
G(T, D, N)=\min _{\eta_{z}} \Phi\left(T, D, N ; \eta_{z}\right)
$$

No ponto estacionário de $\Phi$, o parâmetro $m_{z}$ coincide com a magnetização por spin na camada $z$.

\section{$3.3 \quad$ O limite $T \rightarrow 0$}

Para $\beta=1 / k T \rightarrow \infty$, as equações de campo médio (3.19) e (3.20), e o funcional da energia livre (3.27) ficam, respectivamente,

$$
\begin{gathered}
m_{z}=\frac{\operatorname{sgn}\left(\eta_{z}\right)}{2}\left[1+\left\langle\operatorname{sgn}\left(\left|\eta_{z}\right|-D\right)\right\rangle_{c}\right] \\
\eta_{z}=4 J_{0} m_{z}+J_{1}\left(m_{z+1}+m_{z-1}\right)+J_{2}\left(m_{z+2}+m_{z-2}\right),
\end{gathered}
$$

e

$$
N^{-3} \Phi=\left\langle\frac{D}{2}[1-\operatorname{sgn}(D)]\right\rangle_{c}+\frac{1}{N} \sum_{z} \int_{0}^{m_{z}} \eta_{z} d m_{z}
$$




$$
\begin{aligned}
& -\frac{1}{2 N} \sum_{z}\left[4 J_{0} m_{z}^{2}+J_{1} m_{z}\left(m_{z+1}+m_{z-1}\right)\right. \\
& \left.+J_{2} m_{z}\left(m_{z+2}+m_{z-2}\right)\right] .
\end{aligned}
$$

Nas equações (3.29) e (3.31), $\operatorname{sgn}(x)$ é a função sinal

$$
\operatorname{sgn}(x)=\left\{\begin{array}{rl}
+1 & x>0 \\
0 & x=0 \\
-1 & x<0
\end{array}\right.
$$

\subsection{Expansão de Landau}

Para estudarmos as linhas críticas e pontos tricríticos do modelo, nesta secção desenvolvemos a expansão de Landau (Kincaid e Cohen, 1975) do funcional da energia livre (3.27). Todos os resultados obtidos são válidos para uma distribuição de probabilidades genérica do campo cristalino. Inicialmente notamos que o funcional (3.27) não está escrito explicitamente em termos de $m_{z}$, uma vez que o funcional depende de $\eta_{z}$. Para escrevermos a equação (3.27) como uma função de $m_{z}$, expandimos o lado direito da equação (3.19) na forma

$$
m_{z}=a_{1} \eta_{z}+a_{3} \eta_{z}^{3}+a_{5} \eta_{z}^{5}+\cdots
$$

onde

$$
\begin{aligned}
& a_{1}=\beta\left\langle\frac{1}{\left.1+\frac{1}{2} e^{\beta D}\right\rangle_{c}}\right. \\
& a_{3}=\frac{\beta^{3}}{6}\left\langle\frac{1}{1+\frac{1}{2} e^{\beta D}}-\frac{3}{\left(1+\frac{1}{2} e^{\beta D}\right)^{2}}\right\rangle_{c} \\
& a_{5}=\frac{\beta^{5}}{120}\left\langle\frac{1}{1+\frac{1}{2} e^{\beta D}}-\frac{15}{\left(1+\frac{1}{2} e^{\beta D}\right)^{2}}+\frac{30}{\left(1+\frac{1}{2} e^{\beta D}\right)^{3}}\right\rangle_{c} .
\end{aligned}
$$

Para invertermos a série de potências e obtermos a expansão

$$
\eta_{z}=b_{1} m_{z}+b_{3} m_{z}^{3}+b_{5} m_{z}^{5}+\cdots
$$


substituímos a equação (3.33) em (3.37) e igualamos os coeficientes de mesma potência em $\eta_{z}$. Obtivemos

$$
\begin{aligned}
& b_{1}=\frac{1}{a_{1}}, \\
& b_{3}=-\frac{a_{3}}{a_{1}^{4}}, \\
& b_{5}=3 \frac{a_{3}^{2}}{a_{1}^{7}}-\frac{a_{5}}{a_{1}^{6}} .
\end{aligned}
$$

Substituindo a expansão (3.37) no funcional (3.27) obtivemos até ordem $m_{z}^{6}$,

$$
\begin{aligned}
N^{-3} \Phi= & -\frac{1}{\beta}\left\langle\ln \left(1+2 e^{-\beta D}\right)\right\rangle_{c}+\frac{b_{1}}{2 N} \sum_{z} m_{z}^{2}+\frac{b_{3}}{4 N} \sum_{z} m_{z}^{4}+\frac{b_{5}}{6 N} \sum_{z} m_{z}^{6} \\
& -\frac{1}{2 N} \sum_{z}\left[4 J_{0} m_{z}^{2}+J_{1} m_{z}\left(m_{z+1}+m_{z-1}\right)\right. \\
& \left.+J_{2} m_{z}\left(m_{z+2}+m_{z-2}\right)\right] .
\end{aligned}
$$

A seguir expandimos a magnetização por spin por camada em uma série de Fourier

$$
m_{z}=\sum_{q} m_{q} e^{i q z}
$$

onde os números de onda $q$ são dados por

$$
q=\frac{2 \pi}{N} k \quad(k \text { inteiro }), \quad-\pi \leq q<\pi
$$

e $m_{q}$ são os coeficientes de Fourier. Neste ponto, chamamos a atenção ao fato de que o número de onda $q$ definido na equação acima difere daquele definido pela equação (2.3) pelo fator $2 \pi$. Substituindo a equação (3.42) em (3.41) obtemos

$$
\begin{aligned}
\Phi= & -\frac{N^{3}}{\beta}\left\langle\ln \left(1+2 e^{-\beta D}\right)\right\rangle_{c}+\frac{N^{2}}{2} \sum_{z} \sum_{q_{1}, q_{2}}\left[b_{1}-J\left(q_{1}\right)\right] m_{q_{1}} m_{q_{2}} e^{i\left(q_{1}+q_{2}\right) z} \\
& +\frac{N^{2} b_{3}}{4} \sum_{z} \sum_{q_{1}, q_{2}, q_{3}, q_{4}} m_{q_{1}} m_{q_{2}} m_{q_{3}} m_{q_{4}} e^{i\left(q_{1}+q_{2}+q_{3}+q_{4}\right) z}
\end{aligned}
$$




$$
+\frac{N^{2} b_{5}}{6} \sum_{z} \sum_{q_{1}, q_{2}, q_{3}, q_{4}, q_{5}, q_{6}} m_{q_{1}} m_{q_{2}} m_{q_{3}} m_{q_{4}} m_{q_{5}} m_{q_{6}} e^{i\left(q_{1}+q_{2}+q_{3}+q_{4}+q_{5}+q_{6}\right) z} .
$$

Supondo que próximo à linha crítica a fase é bem representada por uma magnetização por camada senoidal com número de onda $q_{c}$ e que os harmônicos superiores têm a forma $\left|m_{n q_{c}}\right| \sim\left|m_{q_{c}}\right|^{n}$ (Yokoi et al., 1981), expandimos o funcional da energia livre (3.44) em potências do parâmetro de ordem $\left|m_{q_{c}}\right|$. Obtivemos

$$
N^{-3} \Phi=\Phi_{0}+C_{2}\left|m_{q_{c}}\right|^{2}+C_{4}\left|m_{q_{c}}\right|^{4}+C_{6}\left|m_{q_{c}}\right|^{6}+\cdots
$$

onde

$$
\begin{aligned}
& C_{2}=b_{1}-J\left(q_{c}\right), \\
& C_{4}=\frac{3}{2} b_{3}, \\
& C_{6}=\frac{10}{3} b_{5}-b_{3}^{2}\left[b_{1}-J\left(3 q_{c}\right)\right]^{-1} .
\end{aligned}
$$

No apêndice A a expansão de Landau é desenvolvida em detalhes.

A seguir analisamos os coeficientes da equação (3.45) para obter as linhas críticas e os pontos tricríticos do modelo.

\subsection{Linhas críticas e pontos tricríticos}

Segundo a Teoria de Landau das transições de fases (Kincaid e Cohen, 1975), a linha crítica é dada pela condição

$$
C_{2}=0
$$

a qual, usando a equação (3.46), fornece

$$
b_{1}=J\left(q_{c}\right)
$$


onde $q_{c}$ é o número de onda crítico

$$
q_{c}= \begin{cases}0, & \text { se }-J_{2} / J_{1} \leq 1 / 4, \\ \cos ^{-1}\left(-J_{1} / 4 J_{2}\right), & \text { se }-J_{2} / J_{1}>1 / 4,\end{cases}
$$

e

$$
\begin{aligned}
J\left(q_{c}\right) & =4 J_{0}+2 J_{1} \cos q_{c}+2 J_{2} \cos 2 q_{c} \\
& = \begin{cases}4 J_{0}+2 J_{1}+2 J_{2}, & \text { se }-J_{2} / J_{1} \leq 1 / 4, \\
4 J_{0}-2 J_{2}-J_{1}^{2} / 4 J_{2}, & \text { se }-J_{2} / J_{1}>1 / 4 .\end{cases}
\end{aligned}
$$

Substituindo as equações (3.34) e (3.38) em (3.50), obtivemos a equação da linha crítica

$$
\beta J\left(q_{c}\right)\left\langle\frac{1}{1+\frac{1}{2} e^{\beta D}}\right\rangle_{c}=1 .
$$

A estabilidade da linha crítica é verificada se (Kincaid e Cohen, 1975)

$$
C_{4}>0 \text {. }
$$

Com a equação (3.47), a condição acima pode ser escrita como

$$
b_{3}>0
$$

ou, de acordo com a equação (3.39),

$$
a_{3}<0 .
$$

Entretanto, o coeficiente $a_{3}$ é dado pela equação (3.35). Assim, a condição de estabilidade da linha crítica é

$$
\left\langle\frac{1}{1+\frac{1}{2} e^{\beta D}}\right\rangle_{c}-\left\langle\frac{3}{\left(1+\frac{1}{2} e^{\beta D}\right)^{2}}\right\rangle_{c}<0 .
$$

O ponto tricrítico é determinado pela condição (Kincaid e Cohen, 1975)

$$
C_{2}=C_{4}=0 \text {, }
$$


ou seja, é dado pelas equações (3.53) e

$$
\left\langle\frac{1}{1+\frac{1}{2} e^{\beta D}}\right\rangle_{c}-\left\langle\frac{3}{\left(1+\frac{1}{2} e^{\beta D}\right)^{2}}\right\rangle_{c}=0 .
$$

O ponto tricrítico é estável se, juntamente às equações (3.53) e (3.59), a condição

$$
C_{6}>0
$$

for satisfeita (Kincaid e Cohen, 1975). Então, impondo as condições (3.58) e usando as equações (3.34), (3.35), (3.36), (3.38), (3.39), (3.40) e (3.48), obtivemos a condição de estabilidade do ponto tricrítico

$$
\left\langle\frac{1}{\left(1+\frac{1}{2} e^{\beta D}\right)^{2}}\right\rangle_{c}-\frac{5}{2}\left\langle\frac{1}{\left(1+\frac{1}{2} e^{\beta D}\right)^{3}}\right\rangle_{c}>0 .
$$

Até este ponto nossos cálculos são válidos para uma distribuição de probabilidades genérica do campo cristalino. Para investigarmos a influência da desordem sobre os diagramas de fases do modelo consideramos as distribuições de probabilidades do campo cristalino delta-bimodal (capítulo 4) e gaussiana (capítulo 5). 


\section{Capítulo 4}

\section{Resultados para uma distribuição delta-bimodal}

Neste capítulo consideramos o caso particular de uma distribuição deltabimodal do campo cristalino

$$
P(D)=\frac{1}{2}\left[\delta\left(D-D_{0}-\sigma\right)+\delta\left(D-D_{0}+\sigma\right)\right],
$$

onde o valor médio $D_{0}$ e o desvio padrão $\sigma$ representam, respectivamente, a intensidade e a desordem da anisotropia. Assim, para $\sigma=0$ recuperamos o modelo ANNNI com spin 1 e termo de campo cristalino constante (Cadorin e Yokoi, 1998). Considerando $\sigma \neq 0$, na secção 4.1 construímos diagramas de fases do modelo em $k T / J_{1}=0$. Na secção 4.2 estudamos a região de altas temperaturas do modelo para a obtenção das linhas críticas e pontos tricríticos. Finalmente, na secção 4.3 determinamos as fases moduladas na região de temperaturas intermediárias dos diagramas de fases. 


\subsection{Diagramas de fases em $T=0$}

Considerando a distribuição delta-bimodal (4.1), podemos reescrever a equação de campo médio (3.29) como

$$
m_{z}=\operatorname{sgn}\left(\eta_{z}\right) \begin{cases}1, & \left|\eta_{z}\right|>D_{0}+\sigma \\ \frac{1}{2}, & D_{0}-\sigma<\left|\eta_{z}\right|<D_{0}+\sigma \\ 0, & \left|\eta_{z}\right|<D_{0}-\sigma\end{cases}
$$

onde

$$
\eta_{z}=4 J_{0} m_{z}+J_{1}\left(m_{z-1}+m_{z+1}\right)+J_{2}\left(m_{z-2}+m_{z+2}\right) .
$$

O resultado (4.2) é melhor visualizado no gráfico da figura 4.1.

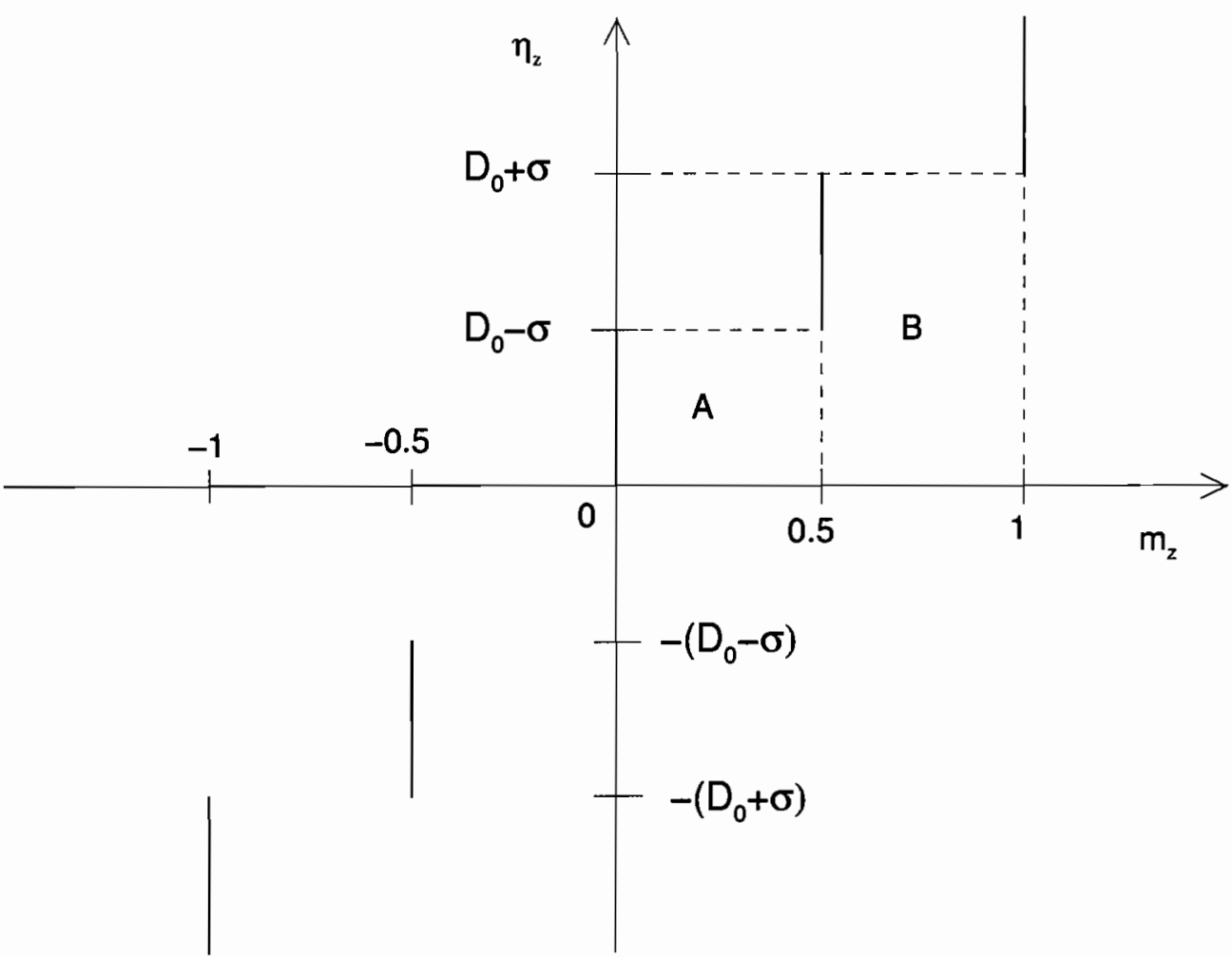

Figura 4.1: Gráfico de $\eta_{z}$ em função de $m_{z}$ construído com o resultado (4.2). 
O funcional da energia livre (3.31) fica

$$
\begin{aligned}
N^{-3} \Phi= & \left\{\frac{1}{4}\left(D_{0}+\sigma\right)\left[1-\operatorname{sgn}\left(D_{0}+\sigma\right)\right]+\frac{1}{4}\left(D_{0}-\sigma\right)\left[1-\operatorname{sgn}\left(D_{0}-\sigma\right)\right]\right\} \\
& +\frac{1}{N} \sum_{z} \int_{0}^{m_{z}} \eta_{z} d m_{z}-\frac{1}{2 N} \sum_{z}\left[4 J_{0} m_{z}^{2}+J_{1} m_{z}\left(m_{z+1}+m_{z-1}\right)\right. \\
& \left.+J_{2} m_{z}\left(m_{z+2}+m_{z-2}\right)\right]
\end{aligned}
$$

podendo ainda ser escrito de uma forma mais simples. Em primeiro lugar, observemos que o termo entre chaves no lado direito dessa equação não depende de $m_{z}$, tendo o mesmo valor para todas as fases. Logo, como comparamos as energias livres das fases moduladas, podemos ignorar esse termo em nossos cálculos. Além disso podemos calcular a integral

$$
y\left(m_{z}\right)=\int_{0}^{m_{z}} \eta_{z} d m_{z}
$$

da equação (4.2). Isso não é difícil de ser feito se observarmos o gráfico da figura 4.1 e notarmos que, segundo a equação (4.2), basta considerar $m_{z}=$ $0, \pm 1 / 2, \pm 1$. Para $m_{z}=0$, temos que $y(0)=0$. Para $m_{z}= \pm 1 / 2, y( \pm 1 / 2)$ corresponde à área da região $A$ da figura 4.1, ou seja,

$$
y\left( \pm \frac{1}{2}\right)=\frac{1}{2}\left(D_{0}-\sigma\right) .
$$

Para $m_{z}= \pm 1, y( \pm 1)$ é a soma das áreas das regiões $A$ e $B$ da figura 4.1, ou seja,

$$
y( \pm 1)=\frac{1}{2}\left(D_{0}+\sigma\right)+\frac{1}{2}\left(D_{0}-\sigma\right)=D_{0} .
$$

Os resultados obtidos acima para $y\left(m_{z}\right)$ estão resumidos na equação abaixo

$$
y\left(m_{z}\right)=\int_{0}^{m_{z}} \eta_{z} d m_{z}= \begin{cases}0, & m_{z}=0 \\ \frac{1}{2}\left(D_{0}-\sigma\right), & m_{z}= \pm \frac{1}{2} \\ D_{0}, & m_{z}= \pm 1\end{cases}
$$


Como y é uma função par e conhecemos cinco pontos dessa função, podemos ajustá-la por um polinômio de grau 4 sem termo independente (para satisfazer $y(0)=0$ ) e apenas com as potências pares (para satisfazer a paridade da função $\left.y\left(m_{z}\right)\right)$. Em outras palavras, podemos escrever $y\left(m_{z}\right)$ na forma conveniente

$$
y\left(m_{z}\right)=a m_{z}^{2}+b m_{z}^{4}
$$

ao invés da expressão ligeiramente mais complicada da equação (4.8). Para determinar os coeficientes $a$ e $b$ da equação (4.9), temos que

$$
y\left(\frac{1}{2}\right)=\frac{1}{2}\left(D_{0}-\sigma\right)=\frac{a}{4}+\frac{b}{16}
$$

e

$$
y(1)=D_{0}=a+b
$$

Resolvendo as equações (4.10) e (4.11) para $a$ e $b$, obtemos

$$
\begin{aligned}
a & =\frac{1}{3}\left(7 D_{0}-8 \sigma\right) \\
b & =\frac{1}{3}\left(-4 D_{0}+8 \sigma\right) .
\end{aligned}
$$

Substituindo os coeficientes $a$ e $b$ dados pela equação (4.12) no polinômio (4.9), o funcional da energia livre à temperatura nula (4.4) é finalmente escrito na forma

$$
\begin{aligned}
N^{-3} \Phi= & \frac{1}{3 N} \sum_{z}\left(7 D_{0}-8 \sigma\right) m_{z}^{2}+\left(-4 D_{0}+8 \sigma\right) m_{z}^{4}-\frac{1}{2 N} \sum_{z}\left[4 J_{0} m_{z}^{2}\right. \\
& \left.+J_{1} m_{z}\left(m_{z+1}+m_{z-1}\right)+J_{2} m_{z}\left(m_{z+2}+m_{z-2}\right)\right] .
\end{aligned}
$$

Para construir o diagrama de fases do modelo em $T=0$, resolvemos as equações (4.2) e (4.3) iterativamente para $1 \leq z \leq N$, com $N \leq 25$. A 
configuração estável é aquela que minimiza o funcional da energia livre (4.13). Todos os diagramas de fases mostrados a seguir foram obtidos para $J_{0}=J_{1}$.

Neste ponto, antes de apresentarmos os diagramas de fases, é importante explicarmos como rotulamos as fases moduladas. Cada fase é representada pelo seu número de onda $\frac{q}{2 \pi}$, seguido por uma letra maiúscula. A letra "A" indica a ausência de planos desordenados na configuração da fase, ao passo que a letra "B" indica a existência de planos desordenados. Com essa notação, uma fase tipo B é parcialmente desordenada. A letra "C" indica que a configuração da fase não apresenta qualquer tipo de simetria, independentemente da existência ou não de planos desordenados. Além disso, de acordo com a equação (4.2), em $T=0$ a magnetização por spin na camada $z\left(m_{z}\right)$ pode assumir apenas cinco valores: $m_{z}=0, \pm \frac{1}{2}, \pm 1$. Por isso, se a configuração de uma dada fase apresentar pelo menos um plano $z$ tal que $\left|m_{z}\right|=\frac{1}{2}$, acrescentamos o símbolo $\left.{ }^{\prime}\right)$ à notação da fase. Por exemplo, observe as configurações das fases e $\frac{1}{4} \mathrm{~A}^{\prime}$ e $\frac{1}{6} \mathrm{~B}^{\prime}$ mostradas nas figuras 4.2 e 4.3 , respectivamente. 


$$
\begin{aligned}
& \mathrm{F}= \\
& F^{\prime}=\Delta \Delta \Delta \Delta \Delta \Delta \Delta \\
& P=-000000 \\
& \frac{1}{4} \mathrm{~A}=\frac{4 \uparrow}{\prod_{i}} \\
& \frac{1}{4} A^{\prime}=\frac{\Delta 4}{\nabla \sigma} \\
& \frac{1}{5} \mathrm{~A}^{\prime}=\frac{\Delta \hat{A}_{\nabla} \Delta}{\prod_{\nabla}} \\
& \frac{1}{5} B^{\prime}=\frac{\Delta I_{\Theta^{\prime}}}{\nabla}
\end{aligned}
$$

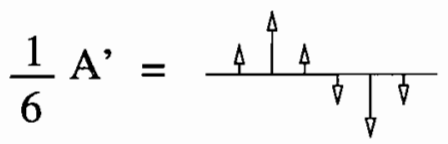

Figura 4.2: Exemplos de fases moduladas encontradas nos diagramas em $T=0$ e as notações correspondentes. 


$$
\begin{aligned}
& \frac{1}{6} B=\operatorname{lin}^{4} \\
& \frac{1}{6} B^{\prime}=-4 \Delta_{\frac{4}{7}}
\end{aligned}
$$

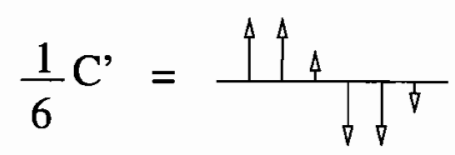

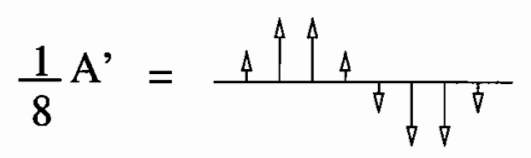

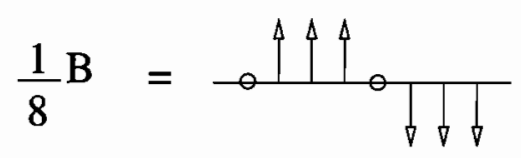

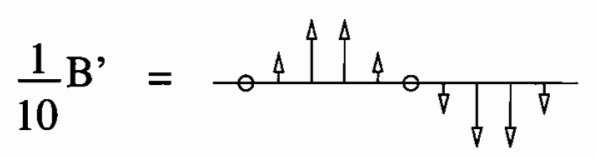

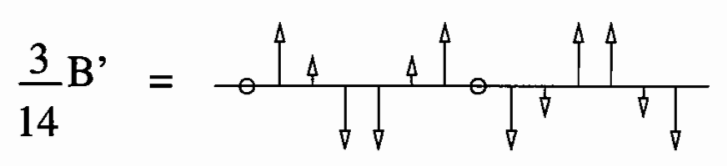

Figura 4.3: Exemplos de fases moduladas encontradas nos diagramas em $T=0$ e as notações correspondentes. 
Na figura 4.4 mostramos o diagrama de fases do modelo em $T=0$ no plano $D_{0} / J_{1}$ versus $-J_{2} / J_{1}$ com $\sigma / J_{1}=0$, que corresponde ao caso de um campo cristalino constante (Cadorin e Yokoi, 1998). Nesse diagrama encontramos as

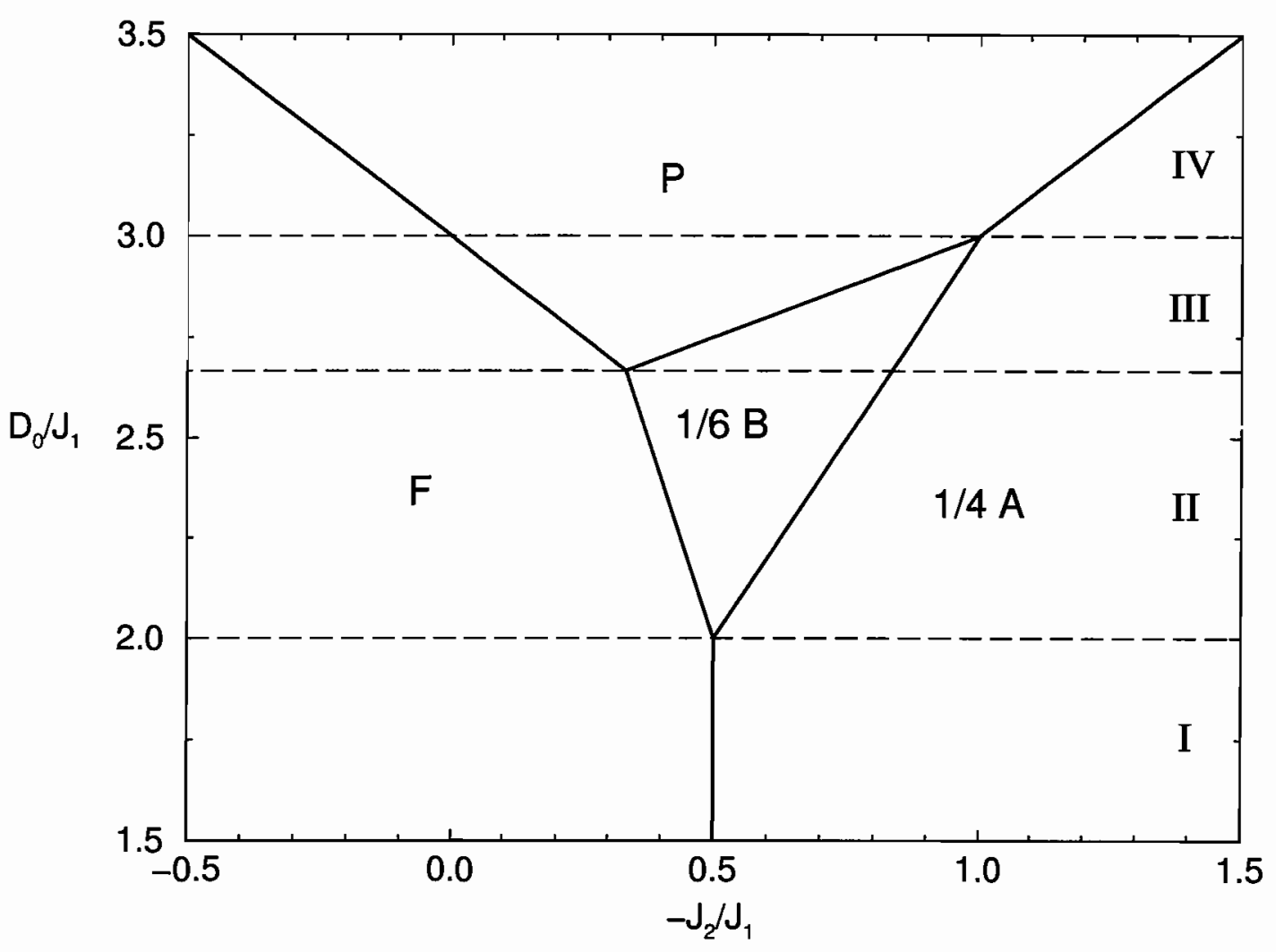

Figura 4.4: Diagrama de fases do modelo em $T=0$, construído para $J_{0}=J_{1}$ e $\sigma / J_{1}=0$, no plano do valor médio $D_{0} / J_{1}$ contra o parâmetro de competição $-J_{2} / J_{1}$. As linhas tracejadas não são transições de fases. Elas apenas delimitam as quatro regiões nas quais o diagrama de fases foi dividido.

fases ferromagnética $(F)$, paramagnética $(P)$, e as fases moduladas $\frac{1}{6} \mathrm{~B}$ e $\frac{1}{4} \mathrm{~A}$ (Cadorin e Yokoi, 1998). Para estudarmos efeitos de desordem, construímos diagramas de fases do modelo no plano $\sigma / J_{1}$ versus $-J_{2} / J_{1}$ para valores fixos de $D_{0} / J_{1}$. Para sermos sistemáticos nesse estudo, dividimos o diagrama de fases nas quatro regiões delimitadas pelas linhas tracejadas mostradas na figura 
4.4. A região I corresponde a $-\infty \leq D_{0} / J_{1} \leq 2$. Nesse intervalo há apenas as fases $\mathrm{F}$ e $\frac{1}{4} \mathrm{~A}$ e o diagrama de fases em $T=0$ nessa região é idêntico ao diagrama de fases do modelo ANNNI usual em $T=0$. Em particular, no limite $D_{0} / J_{1} \rightarrow-\infty$, recobramos o modelo ANNNI usual com spin $\frac{1}{2}$. A região II é delimitada por $2<D_{0} / J_{1} \leq 2.666 \cdots$, onde as fases $F, \frac{1}{4} \mathrm{~A}$ e $\frac{1}{6} \mathrm{~B}$ estão presentes. A região III corresponde a $2.666 \cdots<D_{0} / J_{1}<3$ e nessa região as fases F, P, $\frac{1}{4} \mathrm{~A}$ e $\frac{1}{6} \mathrm{~B}$ estão presentes. Finalmente, na região IV definida por $3 \leq D_{0} / J_{1} \leq \infty$ as fases F, P e $\frac{1}{4} \mathrm{~A}$ estão presentes. No limite $D_{0} / J_{1} \rightarrow \infty$, há apenas a fase paramagnética $(\mathrm{P})$ no diagrama de fases do modelo em $T=0$. Esse fato é confirmado no diagrama da figura 4.4 pelo aumento da largura da fase $\mathrm{P}$ conforme o valor de $D_{0} / J_{1}$ aumenta. Em cada uma das quatro regiões definidas acima fixamos diferentes valores de $D_{0} / J_{1}$, para os quais levantamos diagramas de fases do modelo em $T=0$ no plano $\sigma / J_{1}$ versus $-J_{2} / J_{1}$.

$\mathrm{Na}$ região I consideramos os valores médios $D_{0} / J_{1}=1.5$ e $D_{0} / J_{1}=2$. Os respectivos diagramas no plano $\sigma / J_{1}$ versus $-J_{2} / J_{1}$ estão mostrados nas figuras 4.5 e 4.6. Nesses diagramas, as formas e os tamanhos das fases $\frac{1}{5} \mathrm{~A}^{\prime}$, $\frac{1}{6} \mathrm{~A}^{\prime}, \frac{1}{6} \mathrm{C}^{\prime}$ e $\frac{1}{8} \mathrm{~A}^{\prime}$ são idênticos. Em $T=0$ as fronteiras entre as fases moduladas são funções lineares dos parâmetros $-J_{2} / J_{1}, D_{0} / J_{1}$ e $\sigma / J_{1}$. Portanto, se a região formada por essas fases é idêntica nesses dois valores de $D_{0} / J_{1}$, para qualquer outro valor $D_{0} / J_{1} \leq 2$ continuará sendo. Por outro lado, o ponto onde as fases $\mathrm{F}, \frac{1}{4} \mathrm{~A}$ e $\frac{1}{6} \mathrm{C}^{\prime}$ têm a mesma energia é dado pelas coordenadas $\left(-J_{2} / J_{1}=0.5 ; \sigma / J_{1}=-D_{0} / J_{1}+3\right)$, ou seja, a coordenada $\sigma / J_{1}$ desse ponto aumenta conforme $D_{0} / J_{1}$ diminui. 


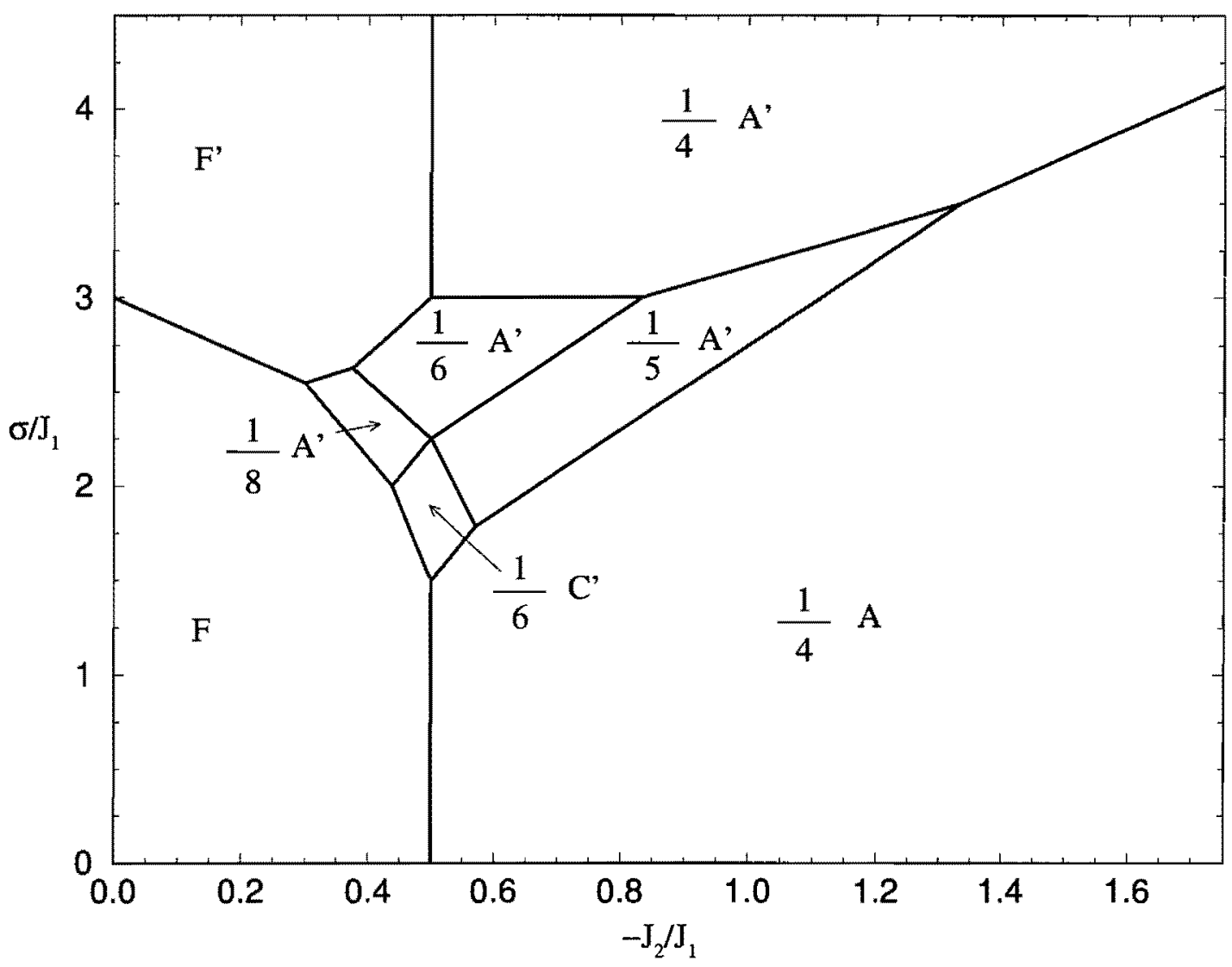

Figura 4.5: Diagrama de fases em $T=0$ no plano $\sigma / J_{1}$ versus $-J_{2} / J_{1}$ para $D_{0} / J_{1}=1.5$. 


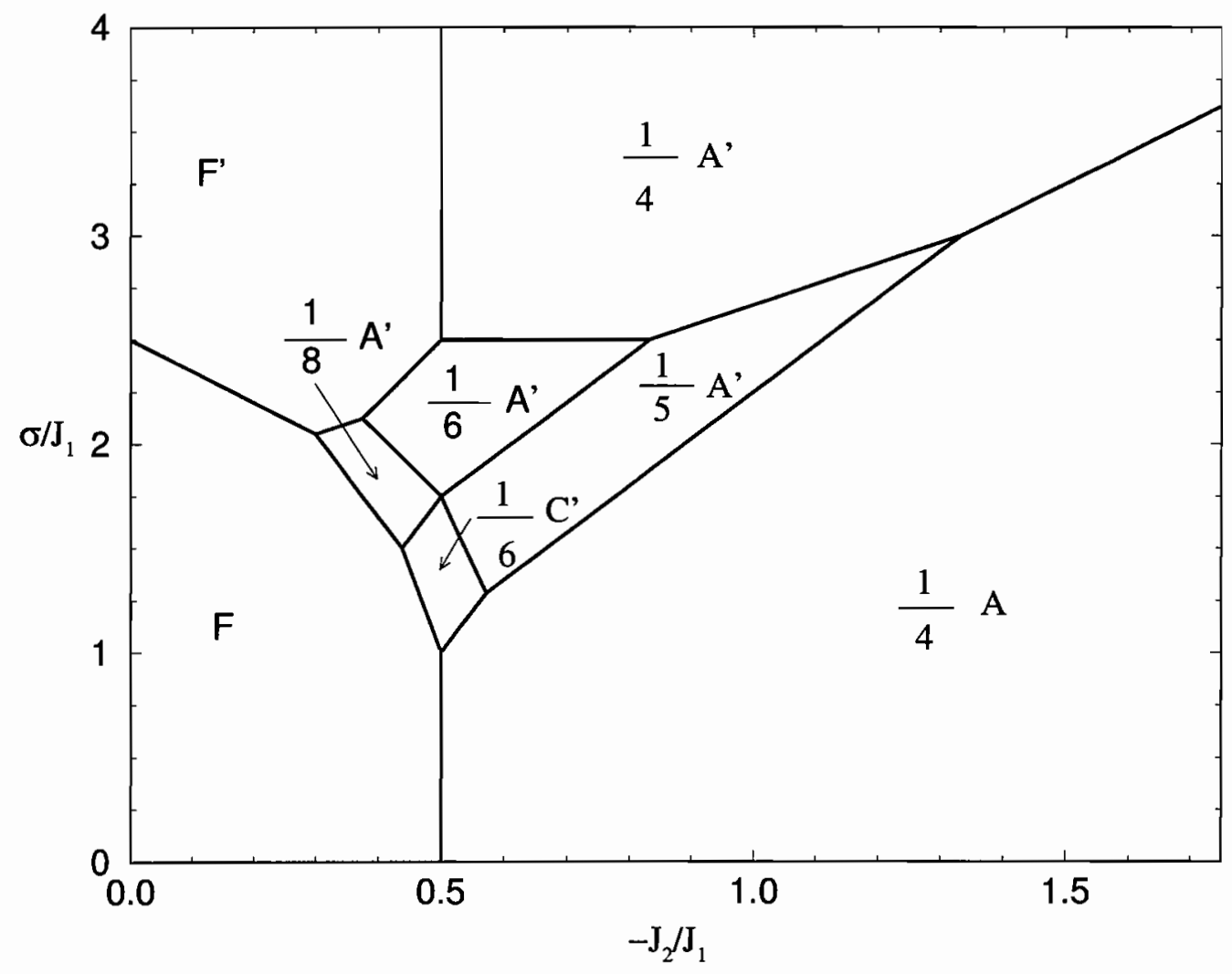

Figura 4.6: Diagrama de fases em $T=0$ no plano $\sigma / J_{1}$ versus $-J_{2} / J_{1}$ para $D_{0} / J_{1}=2$. 
Na região II, construímos os diagramas para $D_{0} / J_{1}=2.1,2.2,2.5$ e 2.6 , mostrados nas figuras $4.7,4.8,4.9$ e 4.10 , respectivamente.

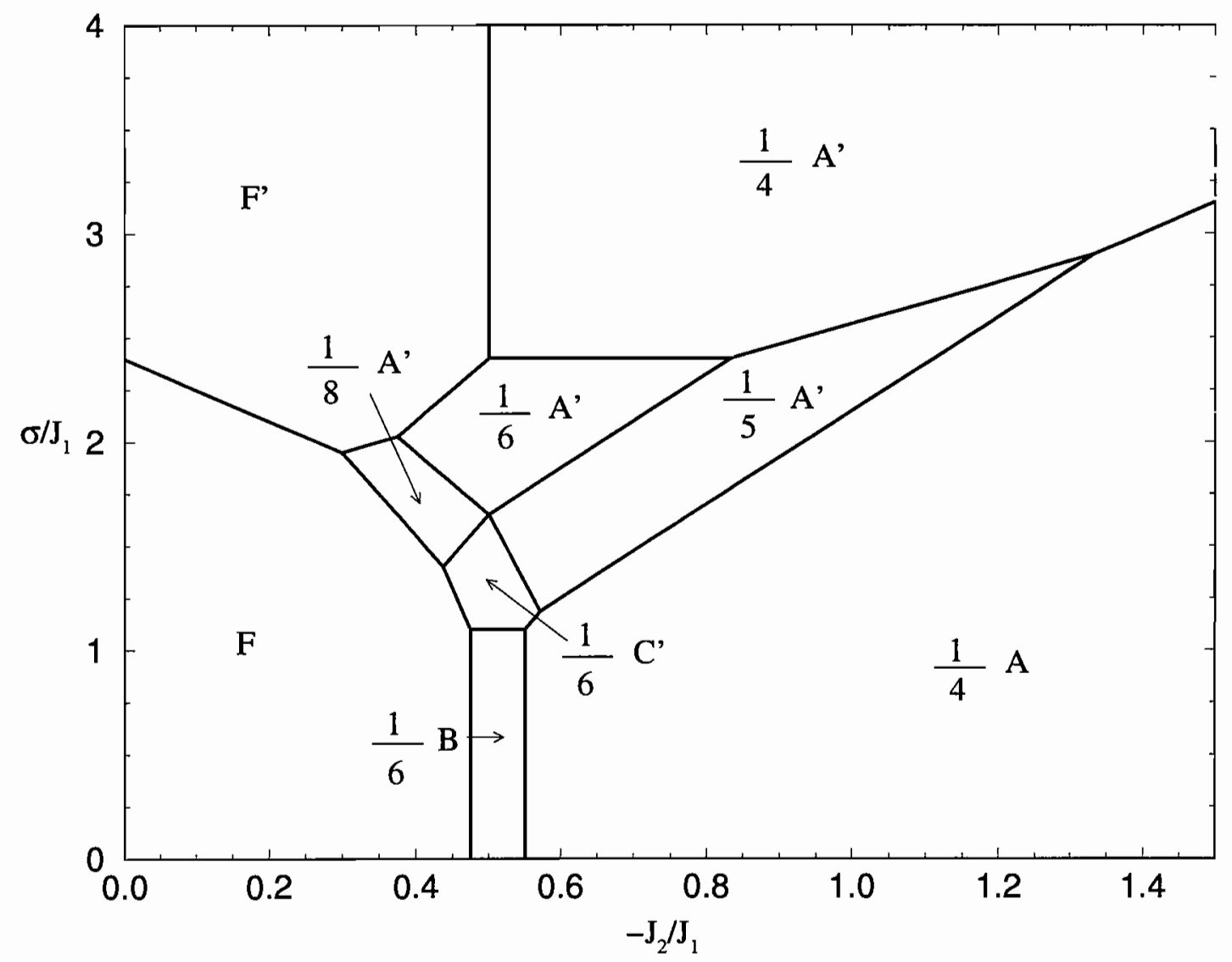

Figura 4.7: Diagrama de fases em $T=0$ no plano $\sigma / J_{1}$ versus $-J_{2} / J_{1}$ para $D_{0} / J_{1}=2.1$.

Na figura 4.7 observamos a existência da fase $\frac{1}{6} \mathrm{~B}$. O surgimento dessa fase é acompanhado pelo início da perda de estabilidade da fase $\frac{1}{6} \mathrm{C}^{\prime}$, ao passo que as fases $\frac{1}{6} \mathrm{~A}^{\prime}, \frac{1}{8} \mathrm{~A}^{\prime}$ e $\frac{1}{5} \mathrm{~A}^{\prime}$ mantém suas regiões de estabilidade inalteradas. Na figura 4.8 vemos que a região de estabilidade da fase $\frac{1}{6} \mathrm{C}^{\prime}$ é ainda menor, enquanto as fases $\frac{1}{6} \mathrm{~A}^{\prime}, \frac{1}{8} \mathrm{~A}^{\prime}$ e $\frac{1}{5} \mathrm{~A}^{\prime}$ permanecem inalteradas e a fase $\frac{1}{6} \mathrm{~B}$ ocupa uma porção maior do diagrama de fases. Para $D_{0} / J_{1}=2.5$ (figura 4.9 ) a fase $\frac{1}{6} \mathrm{C}^{\prime}$ já não está mais presente em $T=0$ e as regiões de estabilidade das fases $\frac{1}{6} \mathrm{~A}^{\prime}, \frac{1}{8} \mathrm{~A}^{\prime}$ e 


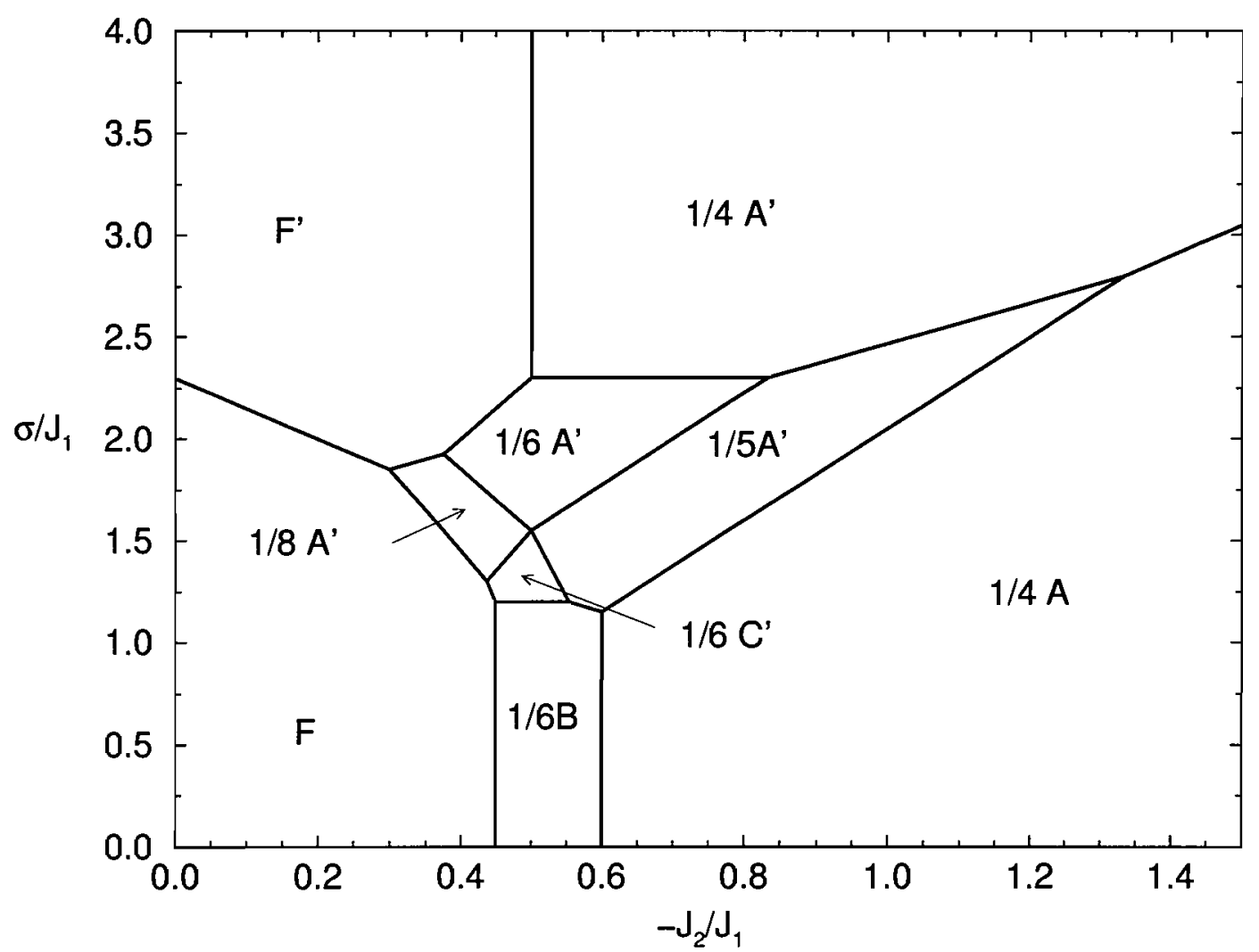

Figura 4.8: Diagrama de fases em $T=0$ no plano $\sigma / J_{1}$ versus $-J_{2} / J_{1}$ para $D_{0} / J_{1}=2.2$.

$\frac{1}{5} A^{\prime}$ foram diminuídas e a fase $\frac{1}{6} B$ se torna estável em uma região ainda maior. Finalmente, as fases presentes no diagrama da figura 4.10 são as mesmas do diagrama anterior. 


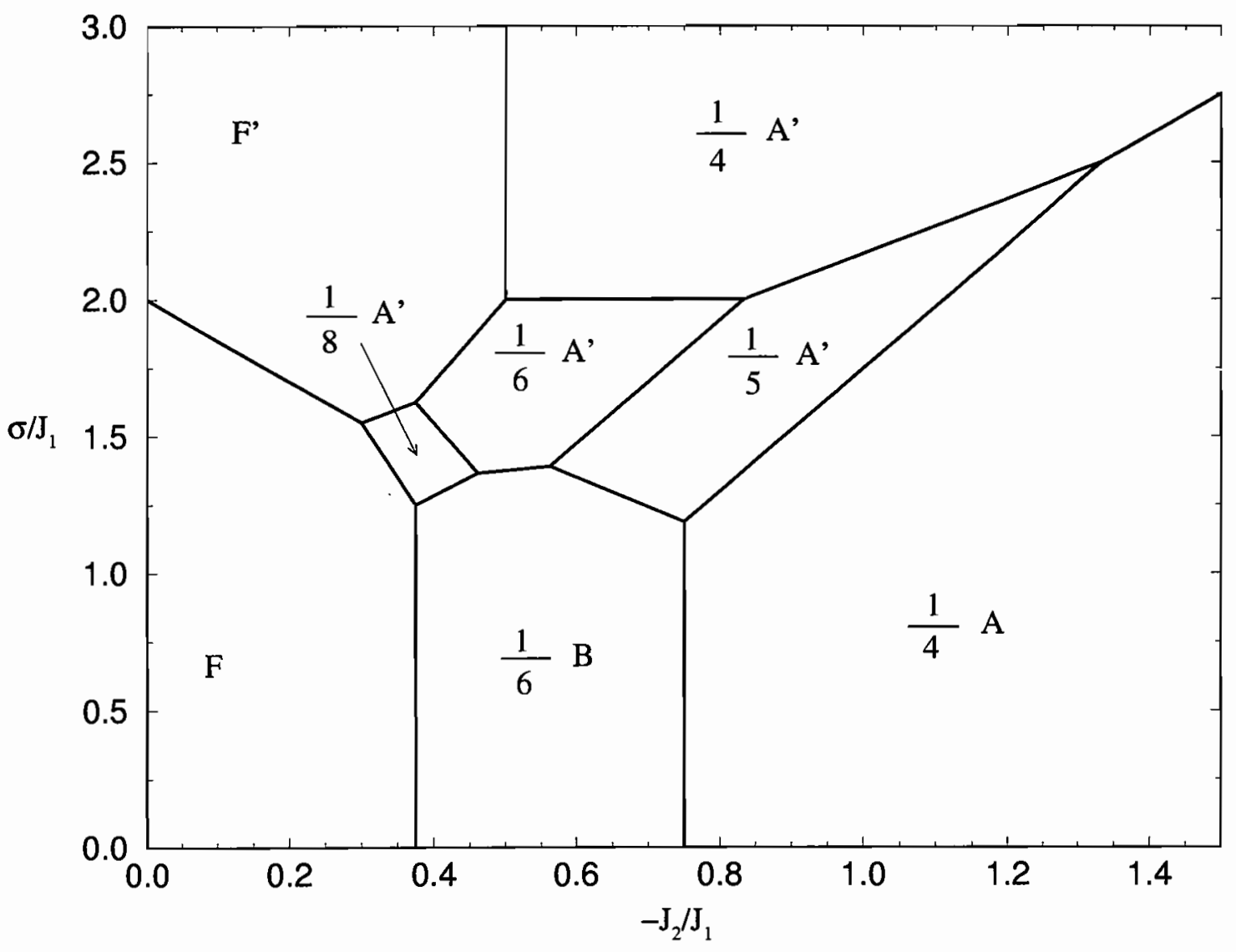

Figura 4.9: Diagrama de fases em $T=0$ no plano $\sigma / J_{1}$ versus $-J_{2} / J_{1}$ para $D_{0} / J_{1}=2.5$. 


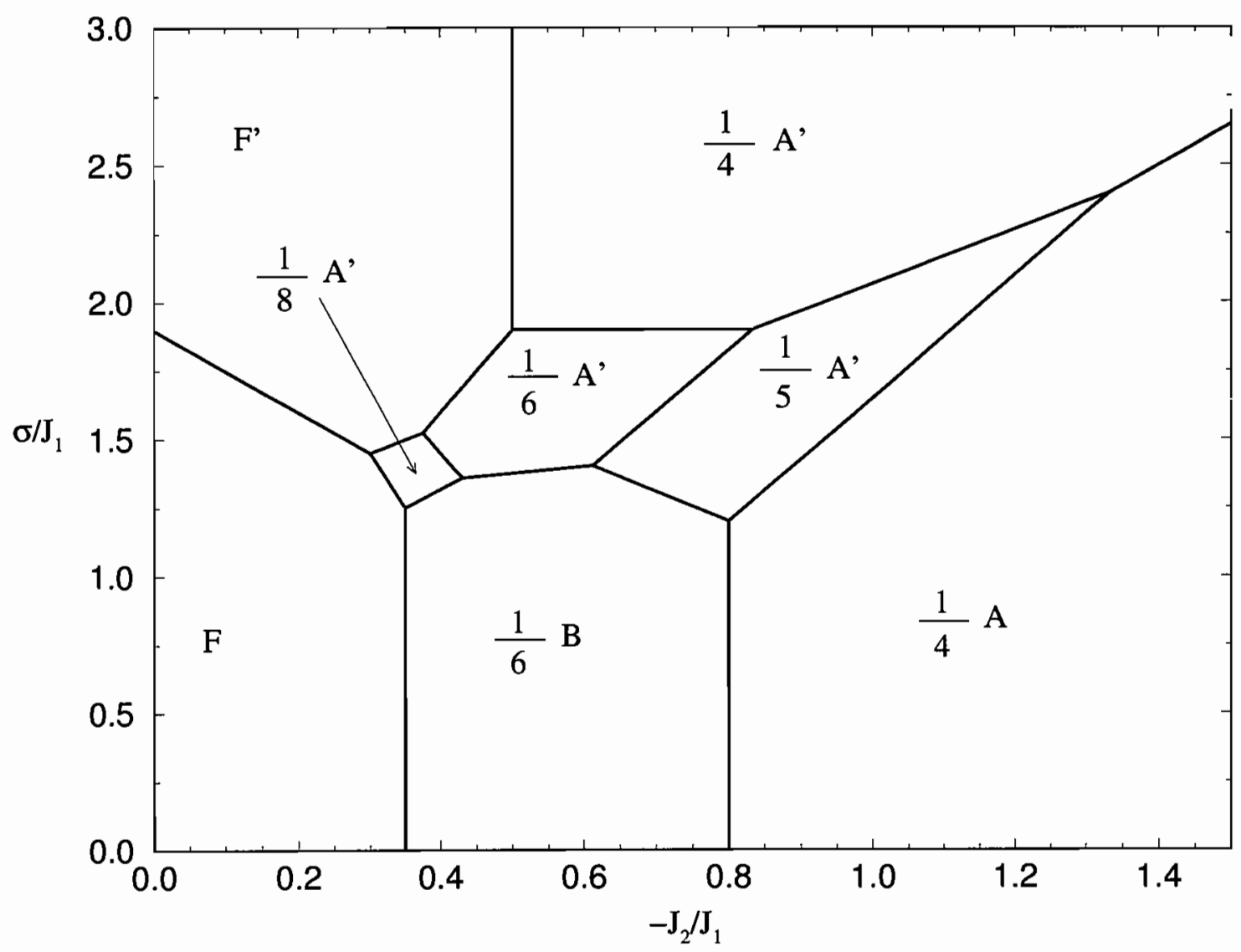

Figura 4.10: Diagrama de fases em $T=0$ no plano $\sigma / J_{1}$ versus $-J_{2} / J_{1}$ para $D_{0} / J_{1}=2.6$. 
$\mathrm{Na}$ região III consideramos os valores médios $D_{0} / J_{1}=2.7$ e 2.75 nas figuras 4.11 e 4.12 , respectivamente. Na figura 4.11 observamos a presença das fases

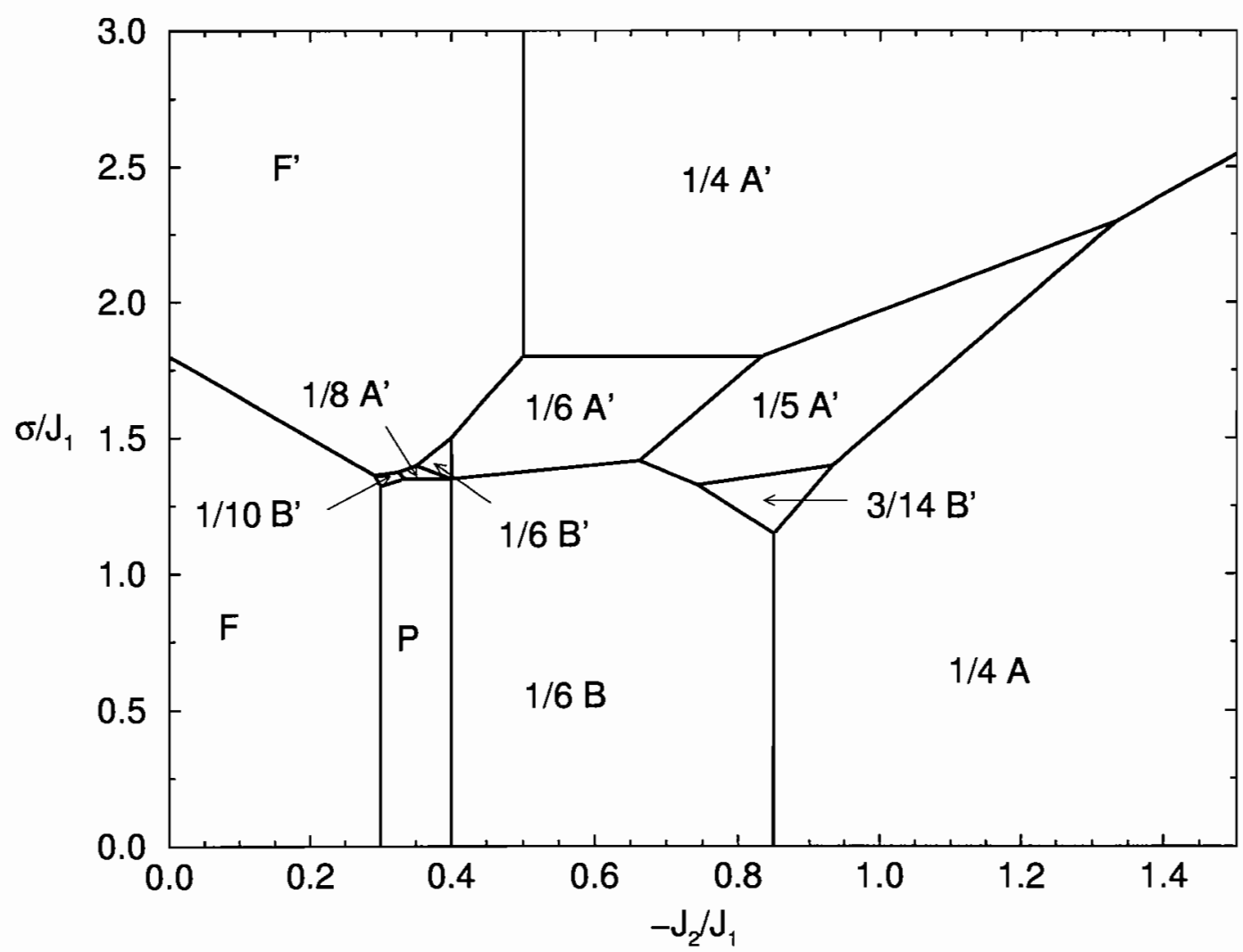

Figura 4.11: Diagrama de fases em $T=0$ no plano $\sigma / J_{1}$ versus $-J_{2} / J_{1}$ para $D_{0} / J_{1}=2.7$.

$\mathrm{P}, \frac{1}{10} \mathrm{~B}^{\prime}, \frac{1}{6} \mathrm{~B}^{\prime}$ e $\frac{3}{14} \mathrm{~B}^{\prime}$. Notamos também um aumento (embora modesto) da região de estabilidade da fase $\frac{1}{6} \mathrm{~B}$ em relação ao diagrama da figura 4.10 e o desfavorecimento da estabilidade das fases $\frac{1}{6} \mathrm{~A}^{\prime}, \frac{1}{8} \mathrm{~A}^{\prime}$ e $\frac{1}{5} \mathrm{~A}^{\prime}$. Para $D_{0} / J_{1}=2.75$ (figura 4.12), as fases $\frac{1}{10} \mathrm{~B}^{\prime}$ e $\frac{1}{8} \mathrm{~A}^{\prime}$ não estão mais presentes e a estabilidade das fases $\frac{1}{5} \mathrm{~A}^{\prime}$ e $\frac{1}{6} \mathrm{~A}^{\prime}$ é desfavorecida ao passo que as fases $\mathrm{P}, \frac{1}{6} \mathrm{~B}^{\prime}, \frac{1}{6} \mathrm{~B}$ e $\frac{3}{14} \mathrm{~B}^{\prime}$ ocupam regiões maiores no diagrama de fases. 


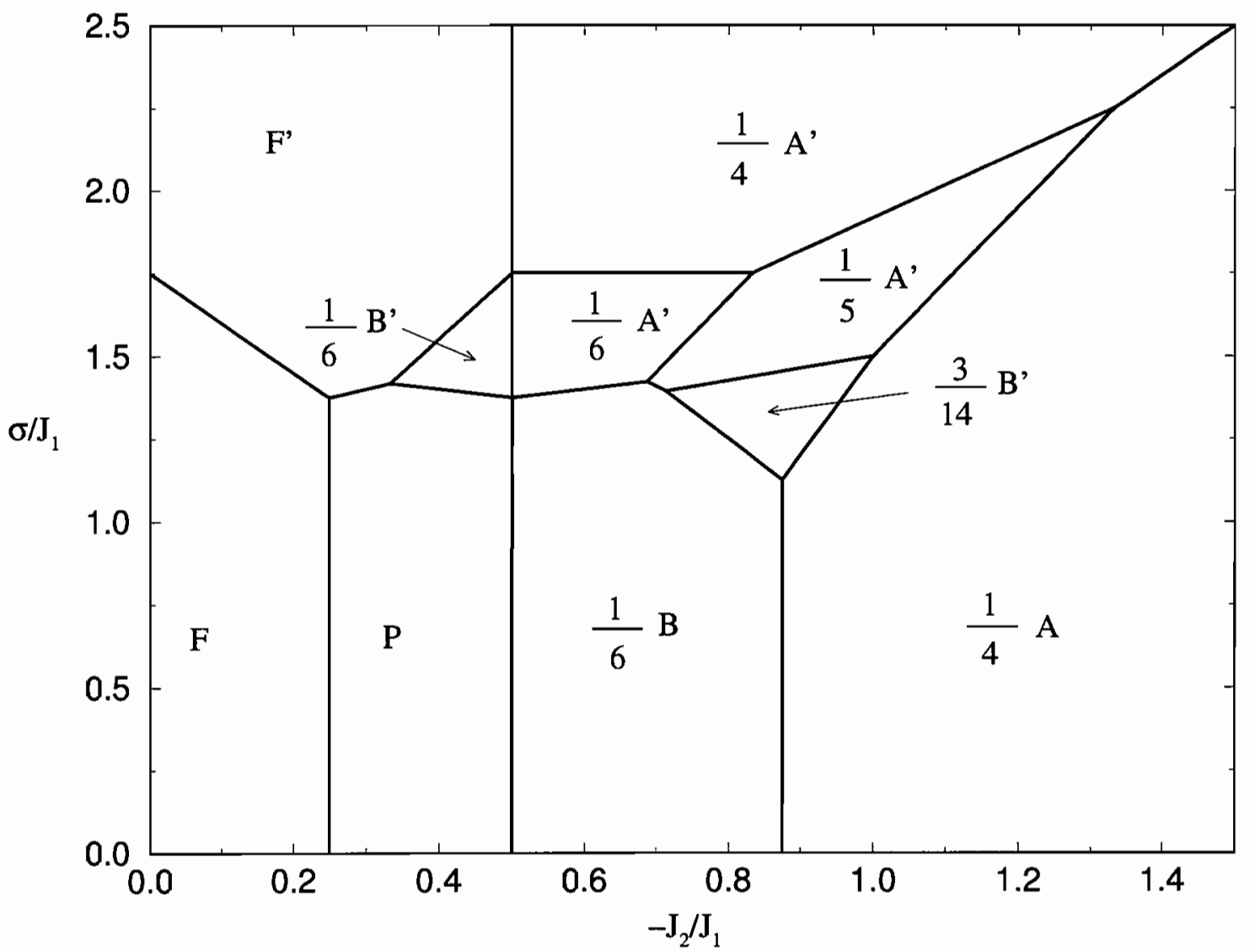

Figura 4.12: Diagrama de fases em $T=0$ no plano $\sigma / J_{1}$ versus $-J_{2} / J_{1}$ para $D_{0} / J_{1}=2.75$. 
Finalmente, na região IV construímos diagramas de fases do modelo em $T=0$ considerando $D_{0} / J_{1}=3,3.125,4$ e 5 , o que é mostrado nas figuras $4.13,4.14,4.15$ e 4.16 , respectivamente.

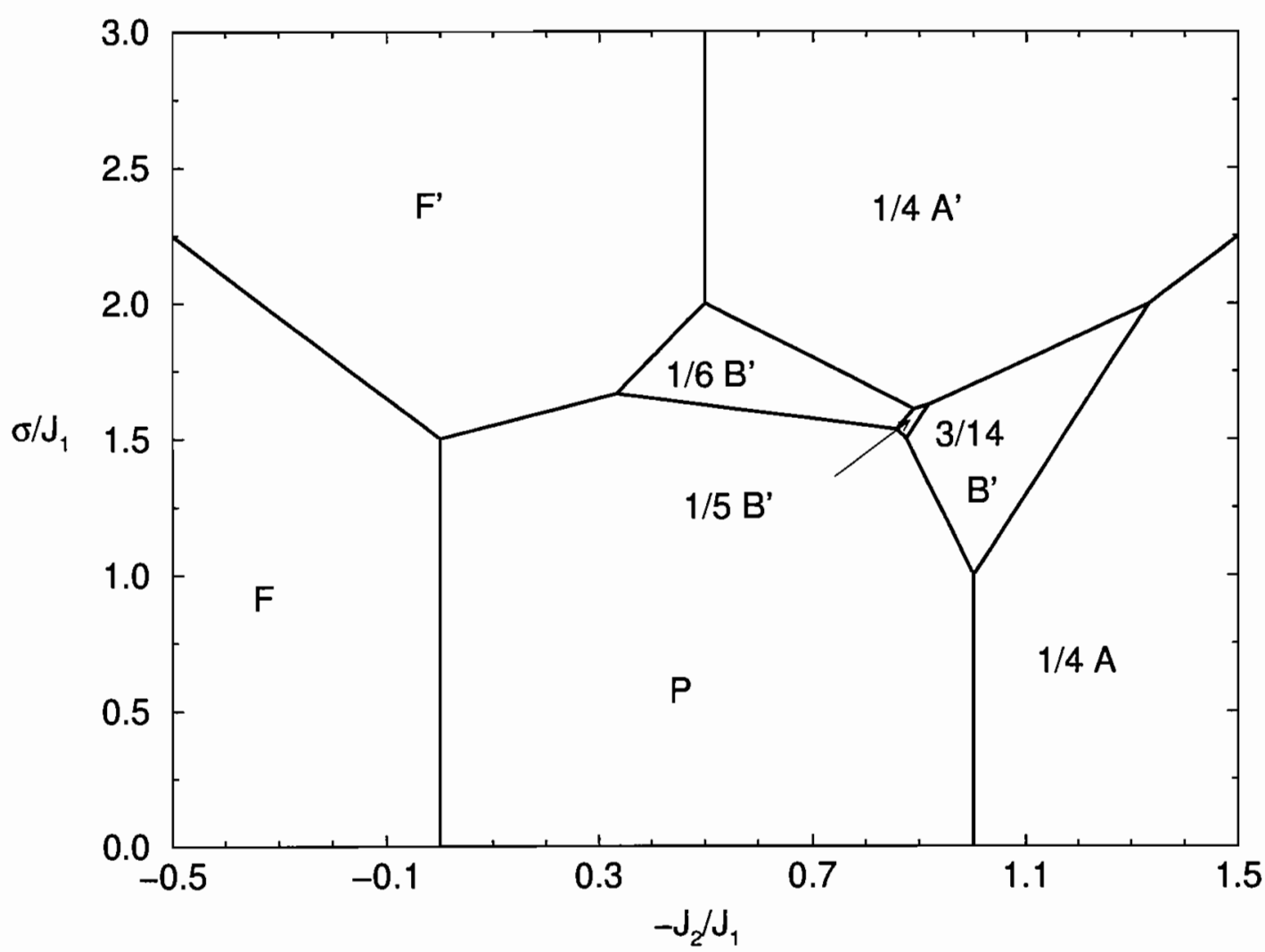

Figura 4.13: Diagrama de fases em $T=0$ no plano $\sigma / J_{1}$ versus $-J_{2} / J_{1}$ para $D_{0} / J_{1}=3$.

Para $D_{0} / J_{1}=3$ (figura 4.13) as fases $\frac{1}{6} \mathrm{~A}^{\prime}, \frac{1}{6} \mathrm{~B}$ e $\frac{1}{5} \mathrm{~A}^{\prime}$ estão ausentes. As fases $\frac{1}{6} \mathrm{~B}^{\prime}$ e $\frac{3}{14} \mathrm{~B}^{\prime}$ ocupam uma região maior do diagrama e entre elas há uma nova fase presente, $\frac{1}{5} \mathrm{~B}^{\prime}$. Na figura 4.14 , onde $D_{0} / J_{1}=3.125$, observamos o desaparecimento da fase $\frac{1}{5} \mathrm{~B}^{\prime}$ e para $D_{0} / J_{1}=4$ (figura 4.15 ) a fase $\frac{3}{14} \mathrm{~B}^{\prime}$ não está mais presente no diagrama de fases. No diagrama da figura 4.16, correspondente a $D_{0} / J_{1}=5$, vemos que a fase $\frac{1}{6} \mathrm{~B}^{\prime}$ é idêntica (em forma 


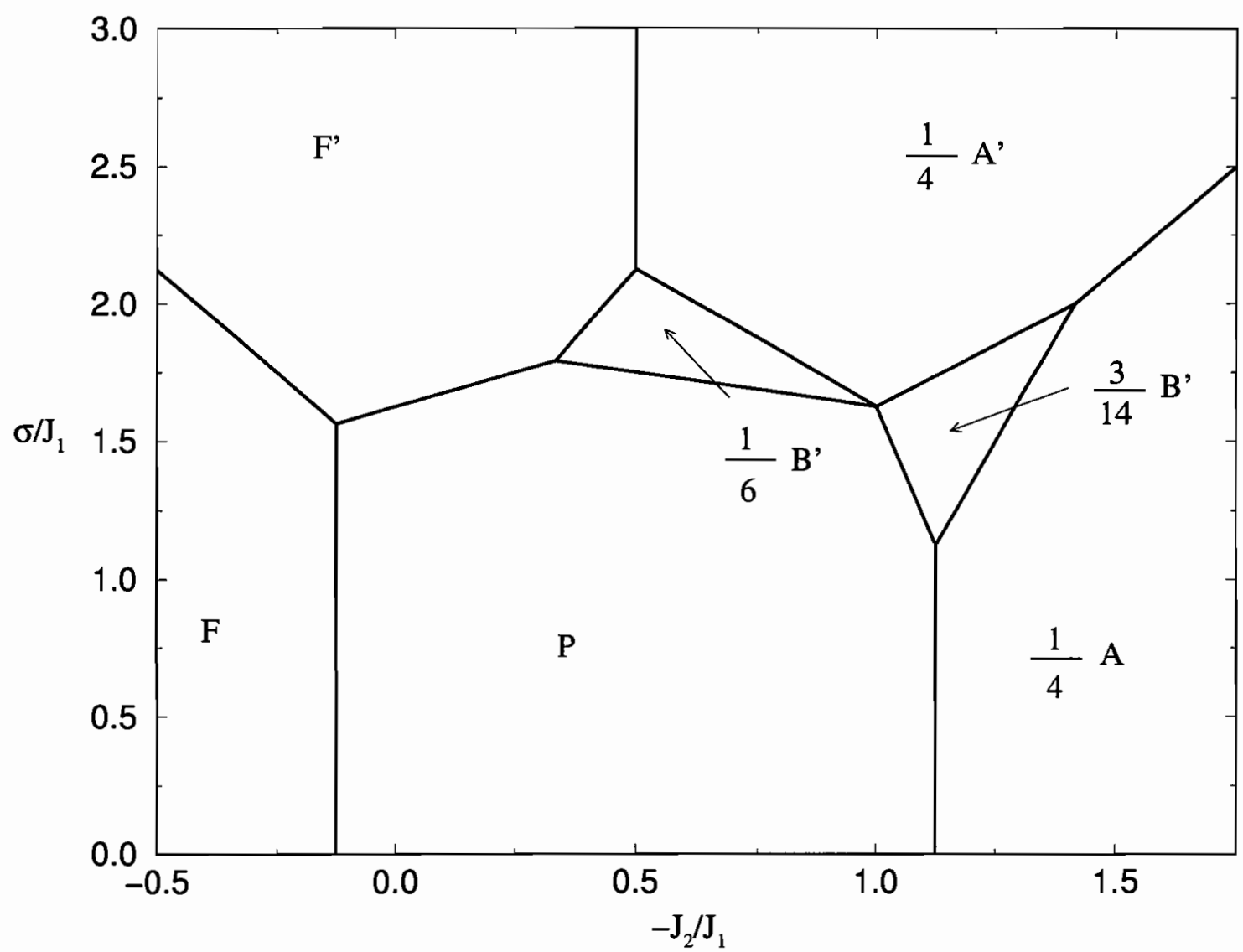

Figura 4.14: Diagrama de fases em $T=0$ no plano $\sigma / J_{1}$ versus $-J_{2} / J_{1}$ para $D_{0} / J_{1}=3.125$.

e tamanho) à obtida na figura 4.15. Como já mencionado anteriormente, as fronteiras das fases são funções lineares dos parâmetros $-J_{2} / J_{1}, D_{0} / J_{1}$ e $\sigma / J_{1}$. Portanto, como essa fase é idêntica para dois valores distintos de $D_{0} / J_{1}$, para qualquer valor $D_{0} / J_{1} \geq 4$ ela continuará sendo e quanto maior o valor de $D_{0} / J_{1}$, maior o valor de $\sigma / J_{1}$ no qual a fase $\frac{1}{6} \mathrm{~B}^{\prime}$ torna-se estável. 


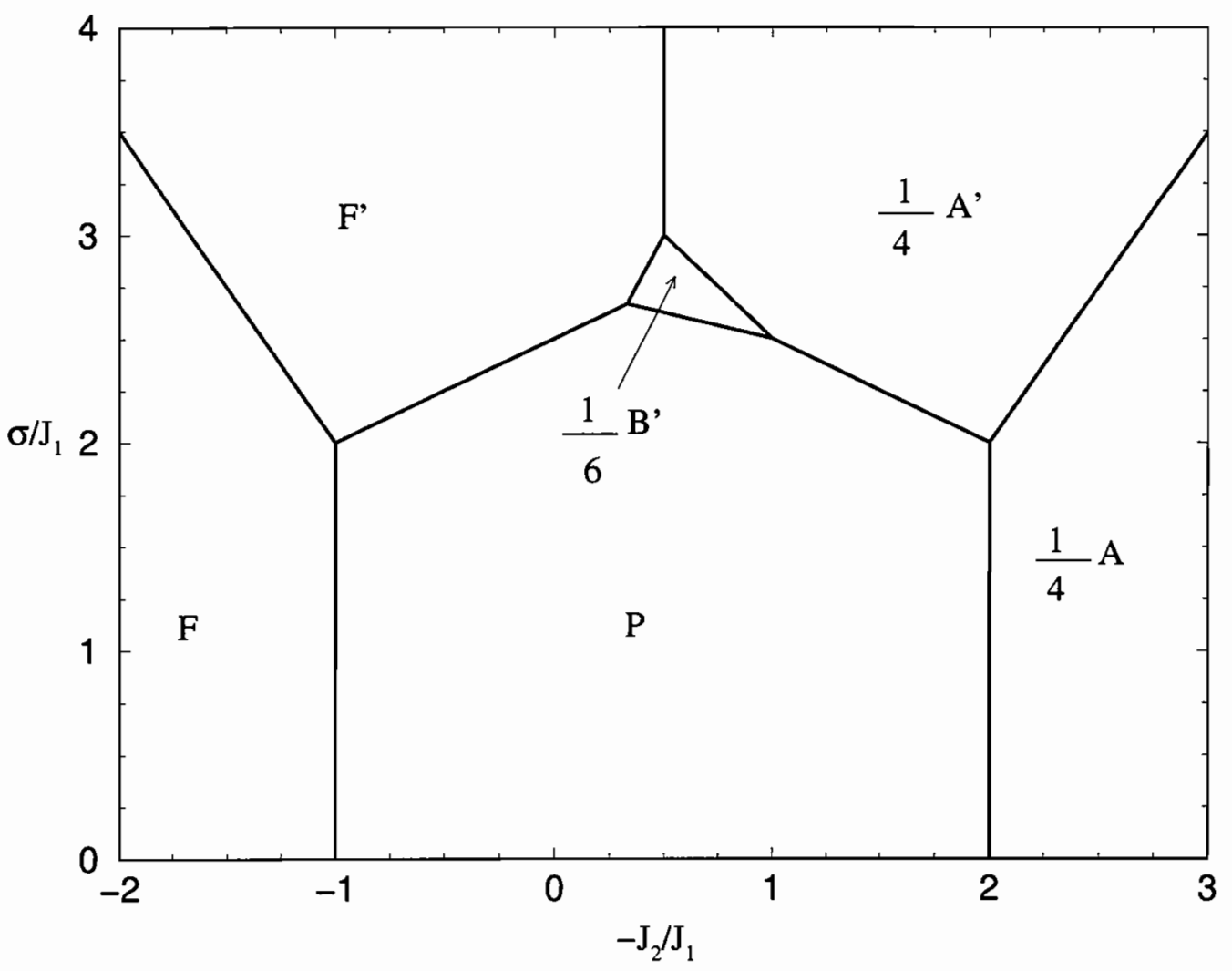

Figura 4.15: Diagrama de fases em $T=0$ no plano $\sigma / J_{1}$ versus $-J_{2} / J_{1}$ para $D_{0} / J_{1}=4$. 


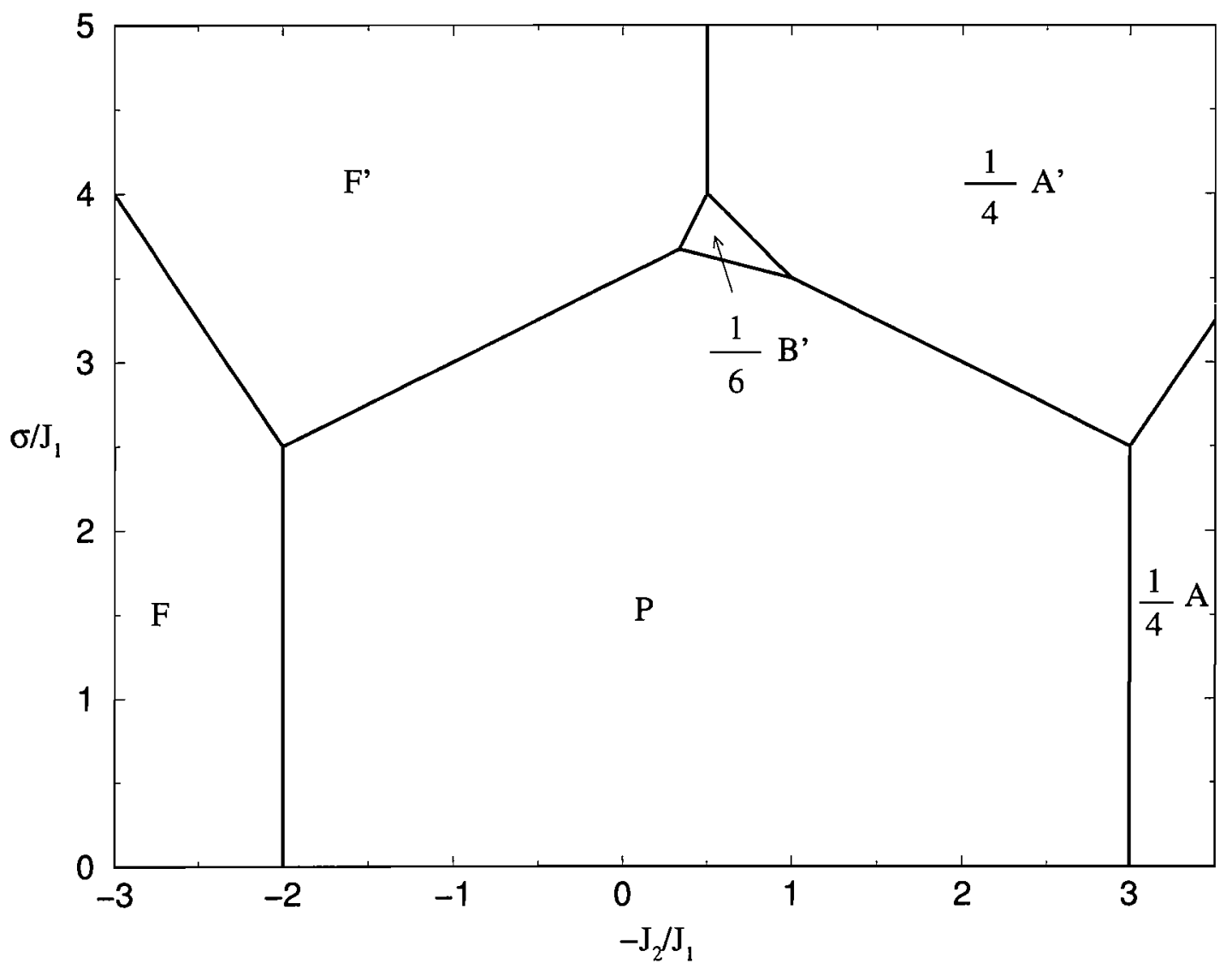

Figura 4.16: Diagrama de fases em $T=0$ no plano $\sigma / J_{1}$ versus $-J_{2} / J_{1}$ para $D_{0} / J_{1}=5$. 


\subsection{Linhas críticas e pontos tricríticos}

Considerando a distribuição de probabilidades delta-bimodal para o campo cristalino (4.1) e $J_{0}=J_{1}$, reescrevemos as equações (3.53) e (3.57) para a linha crítica e sua estabilidade, respectivamente, na forma

$$
\frac{k T_{c}}{J\left(q_{c}\right)}=\frac{1}{2+e^{\left(D_{0}+\sigma\right) / k T_{c}}}+\frac{1}{2+e^{\left(D_{0}-\sigma\right) / k T_{c}}}
$$

para a linha crítica, e

$$
\frac{6}{\left[2+e^{\left(D_{0}+\sigma\right) / k T_{c}}\right]^{2}}+\frac{6}{\left[2+e^{\left(D_{0}-\sigma\right) / k T_{c}}\right]^{2}}-\frac{1}{2+e^{\left(D_{0}+\sigma\right) / k T_{c}}}-\frac{1}{2+e^{\left(D_{0}-\sigma\right) / k T_{c}}}>0
$$

para a estabilidade da linha crítica, onde $J\left(q_{c}\right)$ é dado pela equação (3.52).

No caso da distribuição de probabilidades (4.1), as equações (3.53) e (3.59) definindo os pontos tricríticos e sua condição de estabilidade (3.61), ficam (4.14) e

$\frac{6}{\left[2+e^{\left(D_{0}+\sigma\right) / k T_{c}}\right]^{2}}+\frac{6}{\left[2+e^{\left.\left(D_{0}-\sigma\right) / k T_{c}\right]^{2}}\right.}-\frac{1}{2+e^{\left(D_{0}+\sigma\right) / k T_{c}}}-\frac{1}{2+e^{\left(D_{0}-\sigma\right) / k T_{c}}}=0$,

para os pontos tricríticos e

$$
\frac{1}{\left[2+e^{\left.\left(D_{0}+\sigma\right) / k T_{c}\right]^{2}}\right.}+\frac{1}{\left[2+e^{\left.\left(D_{0}-\sigma\right) / k T_{c}\right]^{2}}\right.}-\frac{5}{\left[2+e^{\left.\left(D_{0}+\sigma\right) / k T_{c}\right]^{3}}\right.}-\frac{5}{\left[2+e^{\left.\left(D_{0}-\sigma\right) / k T_{c}\right]^{3}}\right.}>0
$$

para a estabilidade dos pontos tricríticos.

$\mathrm{Na}$ figura 4.18 mostramos linhas críticas para $\sigma / J_{1}=2$ e diferentes valores médios $D_{0} / J_{1}$. Observamos que o comportamento das linhas críticas é análogo ao caso de anisotropia uniforme $(\sigma=0)$ mostrado na figura 4.17 (Cadorin e Yokoi, 1998). Para $\sigma / J_{1}=2$, observamos que os pontos tricríticos existem 
para $D_{0} / J_{1} \geq 3.2751323 \cdots$. Resolvendo as equações (4.14), (4.16) e (4.17) para outros valores de $D_{0} / J_{1}$, observamos que independentemente do quanto o valor médio $D_{0} / J_{1}$ cresça, sempre haverá um valor do desvio padrão $\sigma / J_{1}$ a partir do qual os pontos tricríticos deixam de existir. A linha no plano $\sigma / J_{1}$ versus $D_{0} / J_{1}$ separando as regiões onde existem ou não pontos tricríticos é mostrada na figura 4.19 .

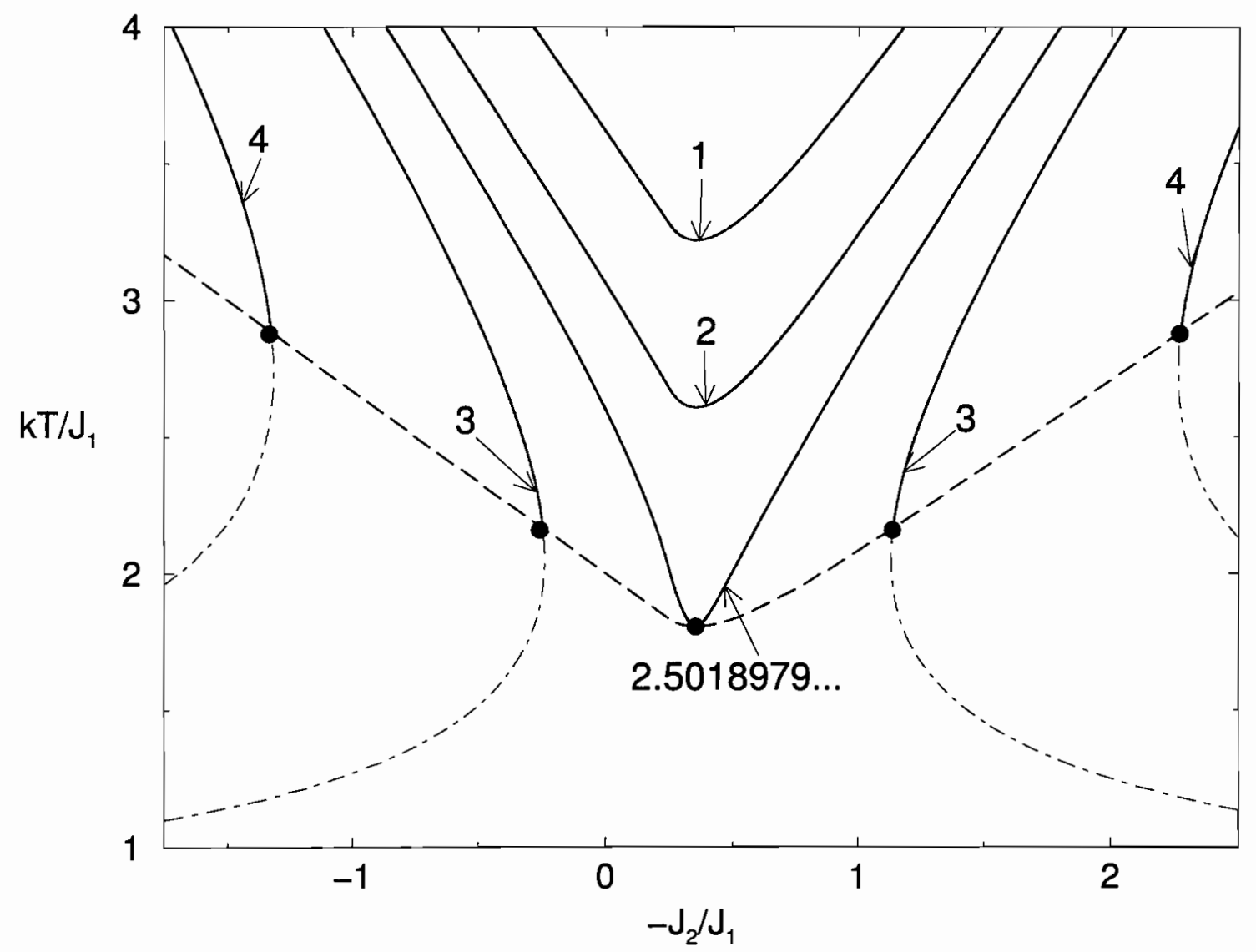

Figura 4.17: Linhas críticas no plano $k T / J_{1}$ versus $-J_{2} / J_{1}$ com $\sigma / J_{1}=0$ e alguns valores de $D_{0} / J_{1}$, representados pelos números associados a cada linha. As linhas contínuas correspondem aos trechos das linhas críticas onde a condição de estabilidade (4.15) é satisfeita, ao passo que os trechos instáveis correspondem às linhas traço-ponto. Para esse valor de $\sigma$ os pontos tricríticos existem apenas para $D_{0} / J_{1} \geq 2.5018979 \cdots$ e estão representados pelos círculos cheios. As linhas tracejada representam os loci dos pontos tricríticos. 


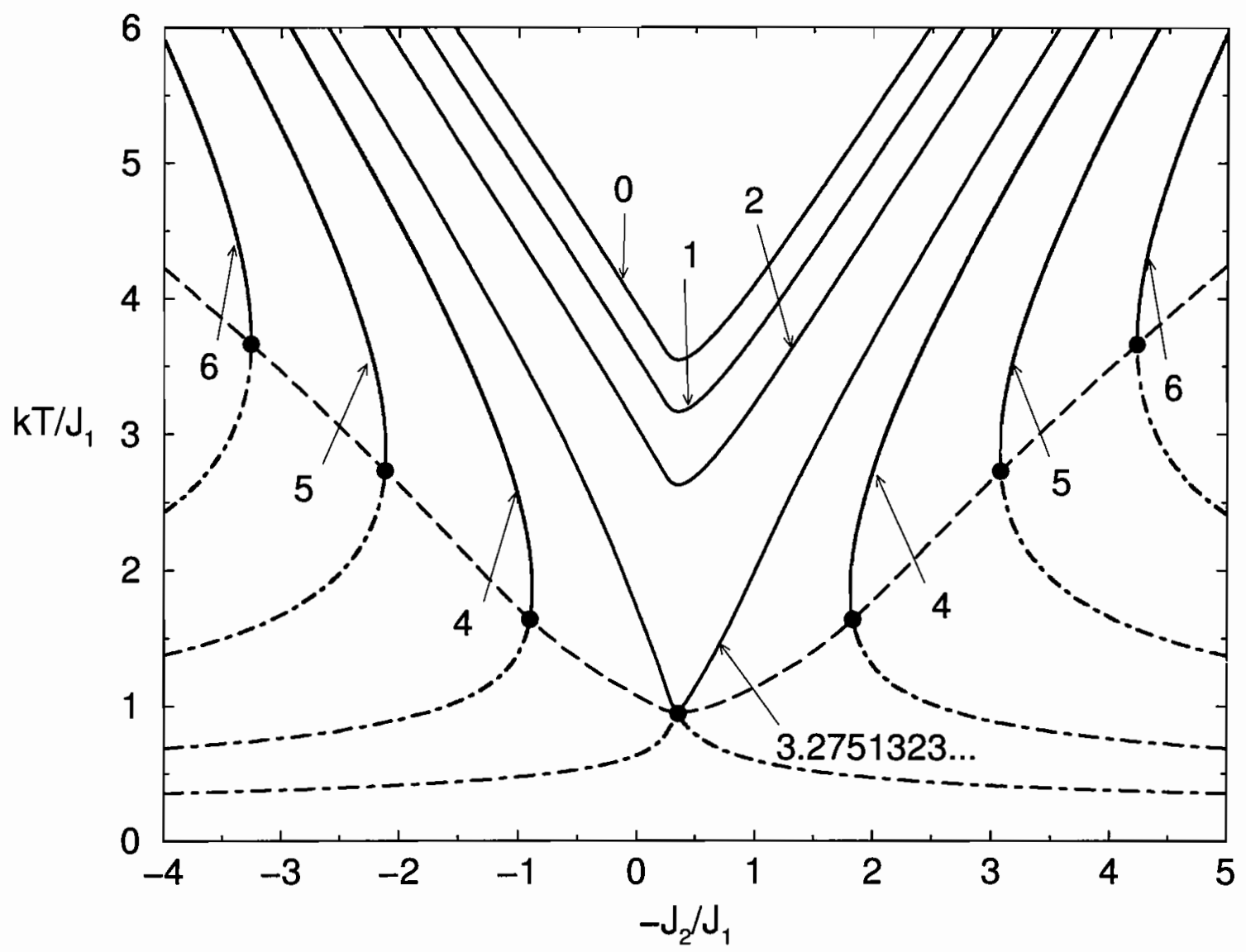

Figura 4.18: Linhas críticas no plano $k T / J_{1}$ versus $-J_{2} / J_{1}$ obtidas para $\sigma / J_{1}=2$. Os valores médios $D_{0} / J_{1}$ considerados estão indicados pelos números associados a cada linha crítica. As linhas contínuas correspondem às porções das linhas críticas onde a condição de estabilidade (4.15) é satisfeita, enquanto as porções instáveis correspondem às linhas traçoponto. Para esse valor de $\sigma / J_{1}$ os pontos tricríticos existem para $D_{0} / J_{1} \geq 3.2751323 \cdots$, e são representados pelos círculos. As linhas tracejadas representam os loci dos pontos tricríticos. 


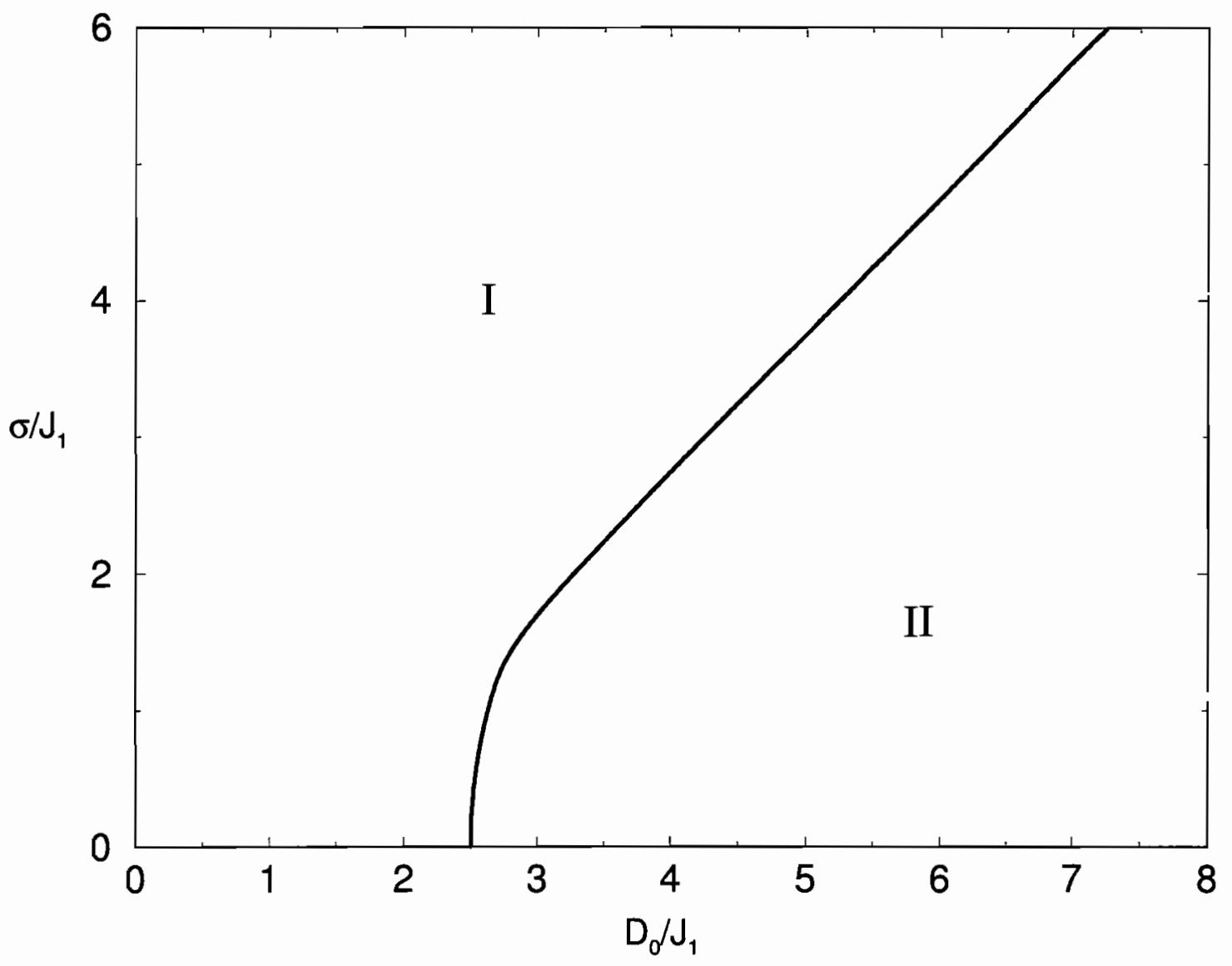

Figura 4.19: Linha que delimita as regiões II e I nas quais, respectivamente, existem e não existem pontos tricríticos. 


\subsection{Diagramas de fases para $T>0$}

Considerando a distribuição delta bimodal (4.1), a equação de campo médio (3.19) e o funcional da energia livre (3.22) ficam

$$
m_{z}=\frac{e^{-\frac{\left(D_{0}+\sigma\right)}{k T}} \sinh \frac{\eta_{z}}{k T}}{1+2 e^{-\frac{\left(D_{0}+\sigma\right)}{k T}} \cosh \frac{\eta_{z}}{k T}}+\frac{e^{\left.-\frac{\left(D_{0}-\sigma\right.}{k T}\right)} \sinh \frac{\eta_{z}}{k T}}{1+2 e^{\left.-\frac{\left(D_{0}-\sigma\right.}{k T}\right)} \cosh \frac{\eta_{z}}{k T}}
$$

e

$$
\begin{aligned}
N^{-3} \Phi= & -\frac{k T}{2} \sum_{z}\left\{\ln \left[1+2 e^{-\frac{\left(D_{0}+\sigma\right)}{k T}} \cosh \frac{\eta_{z}}{k T}\right]+\ln \left[1+2 e^{-\frac{\left(D_{0}-\sigma\right)}{k T}} \cosh \frac{\eta_{z}}{k T}\right]\right\} \\
& +\frac{1}{2 N} \sum_{z}\left[4 J_{1} m_{z}^{2}+J_{1} m_{z}\left(m_{z+1}+m_{z-1}\right)\right. \\
& \left.+J_{2} m_{z}\left(m_{z+2}+m_{z-2}\right)\right]
\end{aligned}
$$

respectivamente, sendo $\eta_{z}$ dado pela equação (4.3).

A seguir mostramos os diagramas de fases do modelo no plano $k T / J_{1}$ versus $-J_{2} / J_{1}$ para $J_{0}=J_{1}$ e alguns valores fixos de $D_{0} / J_{1}$ e $\sigma / J_{1}$. Para cada um desses diagramas consideramos diferentes valores de $\sigma / J_{1}$, mas o mesmo valor médio $D_{0} / J_{1}=2.2$. Isso nos permitiu observar como a desordem influencia o comportamento das fases moduladas. Encontramos as fronteiras das fases moduladas baseados na ocorrência de um processo de ramificação por combinação de estruturas (Selke e Duxbury, 1984). Nesse processo, dadas duas fases com números de onda $P / Q$ e $P^{\prime} / Q^{\prime}$, a fronteira entre essas duas fases é desestabilizada pelo surgimento de uma nova fase entre as duas fases iniciais cujo número de onda é dado pela regra $\left(P+P^{\prime}\right) /\left(Q+Q^{\prime}\right)$, de tal forma que o processo de ramificação pode ser representado por uma sequência de Farey. Assim, o número de fases relevantes cujas energias livres devem ser comparadas para a determinação da fronteira de uma dada fase diminui drasticamente. 
Portanto, esse fato fornece um meio sistemático e eficiente para explorar o diagrama de fases, permitindo considerar-se fases de períodos consideravelmente longos (da ordem de centenas de planos). Baseados nesse método, obtivemos as fases comensuráveis mais proeminentes mostradas nos diagramas expostos a seguir. A notação empregada para rotular as fases moduladas é a mesma utilizada na seç̧ão 4.1. Como $m_{z}$ pode variar continuamente em $T>0$, as fases moduladas ficam representadas apenas pelo seu número de onda seguido pela letra A, B ou C, não sendo necessário empregar a extensão ('). Em todos os diagramas, as linhas separando a fase paramagnética $(\mathrm{P})$ da região ordenada são de segunda ordem. Para tornar claros os comentários que faremos sobre as figuras expostas a seguir, explicamos que todas as comparações citadas em um dado diagrama foram feitas em relação ao diagrama imediatamente anterior, exceto quando explicitamente indicado. O diagrama mostrado na figura 4.20 corresponde ao caso do termo de campo cristalino uniforme e está em concordância com os resultados obtidos anteriormente (Cadorin e Yokoi, 1998). Como já havia sido observado por esses autores, notamos que as fases moduladas sofrem um estreitamento, correspondente à separação entre fases com mesmo número de onda mas com configurações diferentes da magnetização. A configuração da magnetização de cada uma das fases moduladas na região de temperaturas abaixo desse estreitamento é tal que existem planos desordenados (magnetização nula, fases tipo "B") e na região de temperaturas acima dele não existem tais planos (fases tipo "A").

No diagrama de fases obtido para $D_{0} / J_{1}=2.2$ e $\sigma / J_{1}=1$ mostrado na figura 4.21, observamos fases parcialmente desordenadas (tipo B) e o compor- 


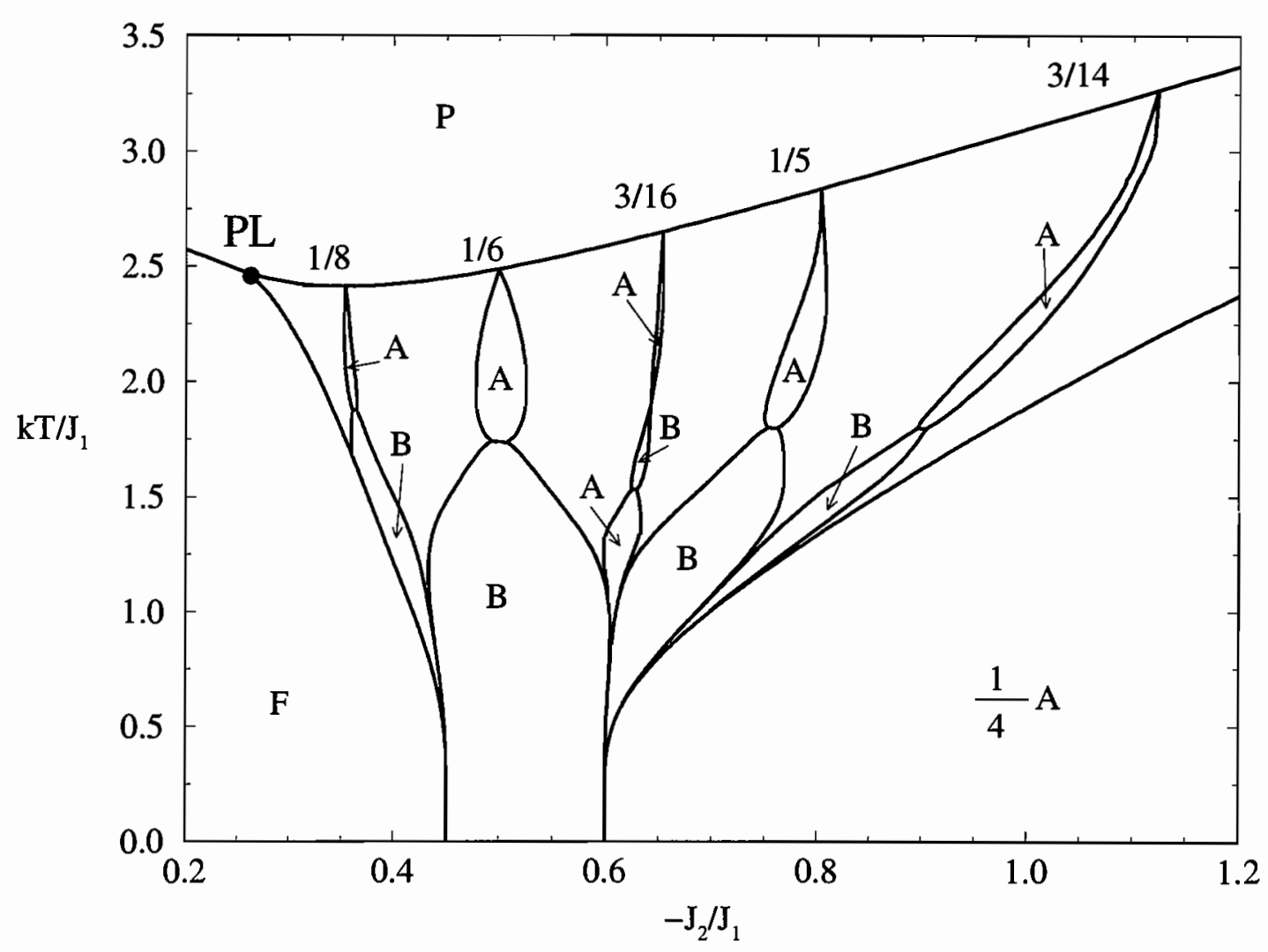

Figura 4.20: Diagrama de fases global do modelo no plano $k T / J_{1}$ versus $-J_{2} / J_{1}$ para $D_{0} / J_{1}=2.2$ e $\sigma / J_{1}=0$ (campo cristalino uniforme).

tamento global desse diagrama é análogo àquele observado para $D_{0} / J_{1}=2.2 \mathrm{e}$ $\sigma=0$ (campo cristalino constante) mostrado na figura 4.20 , revelando que não há alterações qualitativas consideráveis nos diagramas de fases para desordem suficientemente pequena.

Na figura 4.22, onde $\sigma / J_{1}=2$, as fronteiras das fases tornaram-se sinuosas e não existem mais estreitamentos das fases moduladas, podendo-se notar a ausência de fases do tipo B.

Aumentando o desvio padrão para $\sigma / J_{1}=2.25$, observamos na figura 4.23 que em um certo ponto $\left(-J_{2} / J_{1} \approx 0.8, k T / J_{1} \approx 0.5\right)$ do diagrama existe 


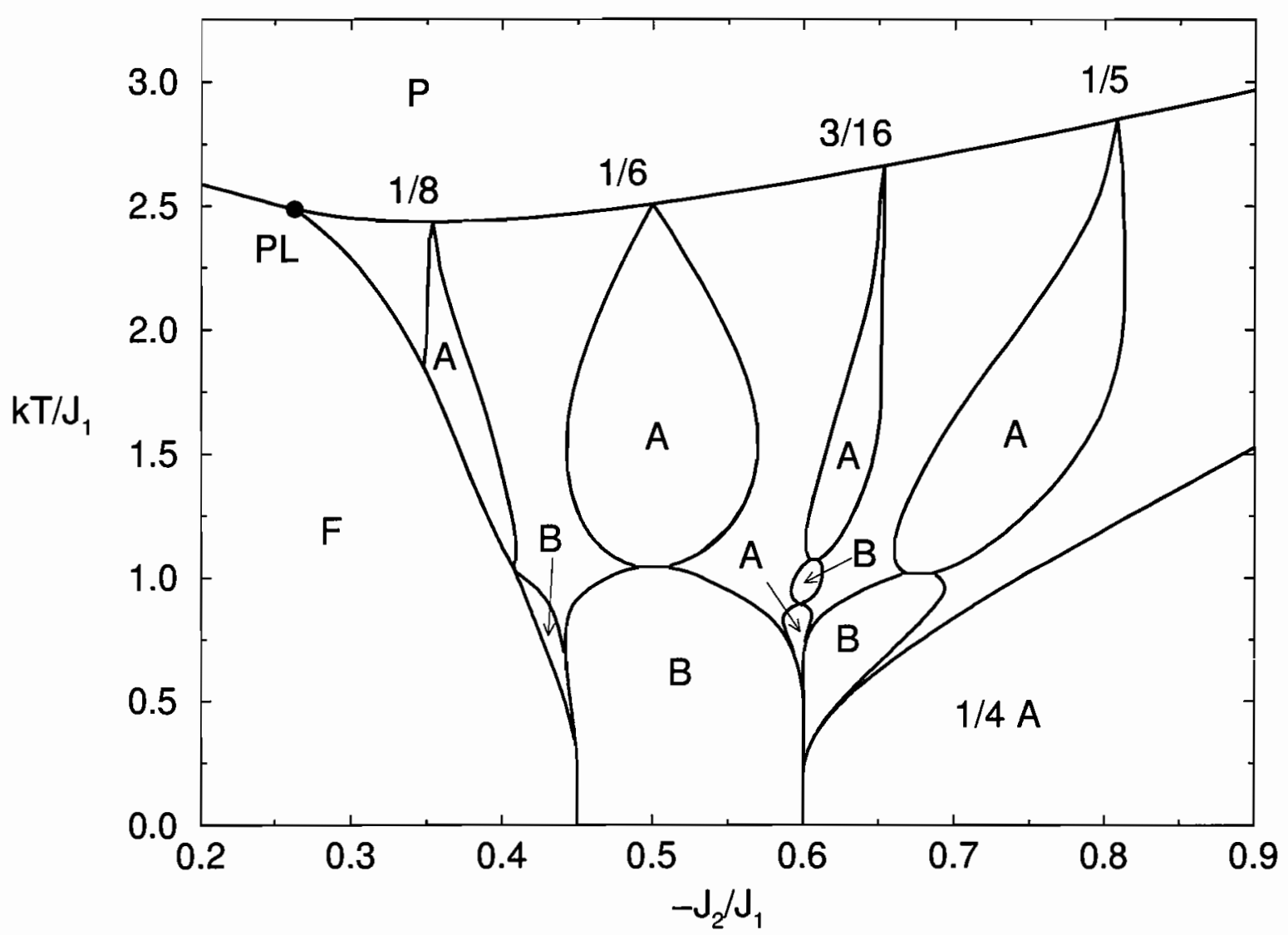

Figura 4.21: Diagrama de fases de campo médio do modelo no plano $k T / J_{1}$ versus $-J_{2} / J_{1}$, construído para $J_{0}=J_{1}, D_{0} / J_{1}=2.2$ e $\sigma / J_{1}=1$. As frações são os números de onda críticos das fases moduladas e as letras maiúsculas indicam a ausência (A) ou a presença (B) de planos desordenados na configuração da fase. A fase paramagnética $(\mathrm{P})$, a fase ferromagnética (F A) e a região modulada se encontram no ponto de Lifshitz (PL).

uma dobra acentuada da fronteira entre as fases $\frac{1}{6} \mathrm{~A}$ e $\frac{1}{5} \mathrm{~A}$. Acreditamos que o "bico" formado por essa dobra seja um ponto superdegenerado ou um ponto de multifases. Além disso, ainda notamos a sinuosidade das fronteiras entre as fases moduladas.

No caso $\sigma / J_{1}=2.3$ (figura 4.24 ), a dobra acentuada entre as fases $\frac{1}{6} \mathrm{~A}$ e $\frac{1}{5} \mathrm{~A}$ ainda existe, mas muito próximo a $T=0$. A sinuosidade das fronteiras permanece. 


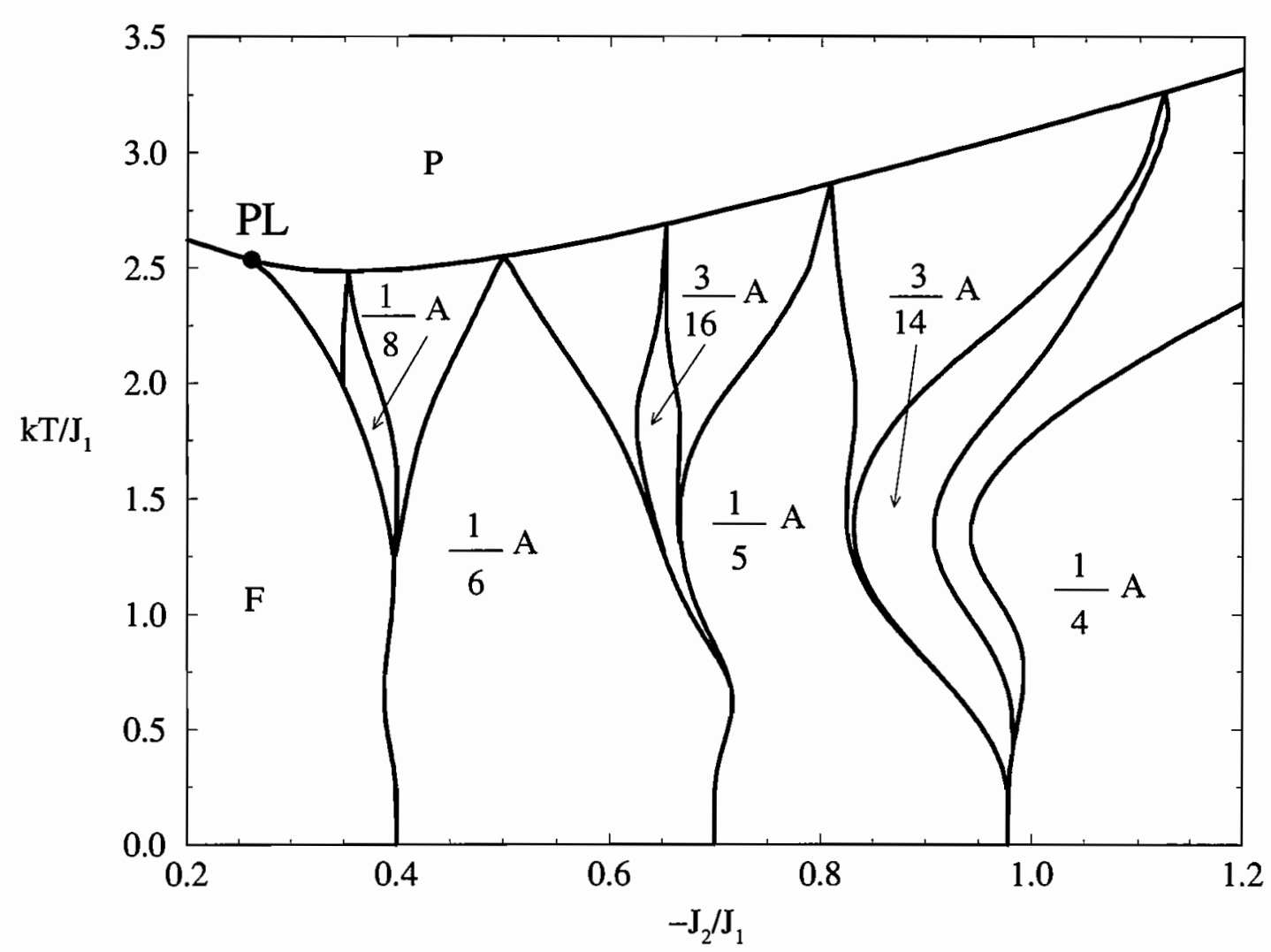

Figura 4.22: Diagrama de fases global do modelo no plano $k T / J_{1}$ versus $-J_{2} / J_{1}$ para $D_{0} / J_{1}=2.2$ e $\sigma / J_{1}=2$.

Para $\sigma / J_{1}=2.31$ o diagrama sofre uma mudança notável na região de baixas temperaturas, conforme mostrado na figura 4.25. A $T=0$, a fase $\frac{1}{6} \mathrm{~A}$ não está presente em um intervalo de valores de $-J_{2} / J_{1}$, mas apenas no ponto $\left(-J_{2} / J_{1}=0.5, k T / J_{1}=0\right)$. A fase $\frac{1}{4} \mathrm{~A}$ é estável a $T=0$ em duas regiões: uma dessas regiões está limitada a uma porção do diagrama onde o parâmtero $-J_{2} / J_{1}$ varia entre 0.5 e 0.85 e a temperatura $k T / J_{1}$ entre 0 e 0.25 , aproximadamente, e está separada da outra região onde a fase $\frac{1}{4} \mathrm{~A}$ não é limitada. Além disso existe uma dobra acentuada em cada uma das suas fronteiras com as fases $\frac{1}{6} \mathrm{~A}$ e $\frac{1}{5} \mathrm{~A}$. Para temperaturas mais altas, o comportamento do diagrama 


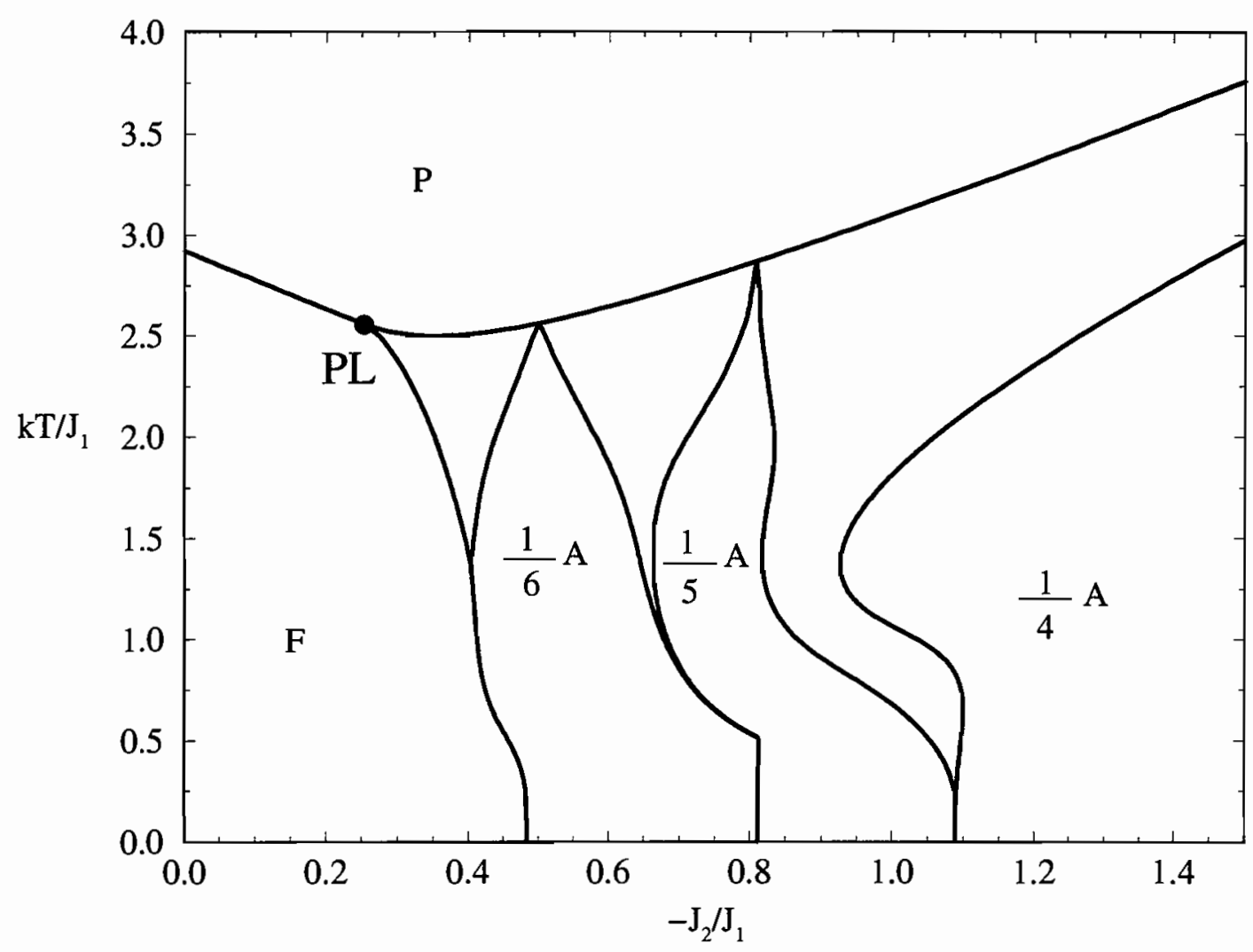

Figura 4.23: Diagrama de fases global do modelo no plano $k T / J_{1}$ versus $-J_{2} / J_{1}$ para $D_{0} / J_{1}=2.2$ e $\sigma / J_{1}=2.25$.

não foi alterado.

$\mathrm{Na}$ figura 4.26 mostramos o diagrama de fases obtido para $D_{0} / J_{1}=2.2$ and $\sigma / J_{1}=2.45$. Observamos apenas fases do tipo A. Para esse valor de $\sigma / J_{1}$ as regiões de ocorrência da fase $1 / 4 \mathrm{~A}$ se uniram e o diagrama de fases passou a consistir de duas regiões moduladas separadas pela fase 1/4A. Em uma dessas regiões as fases moduladas surgem do ponto de multifases de coordenadas $\left(-J_{2} / J_{1}=0.5 ; k T / J_{1}=0\right)$. O comportamento global dessa porção do diagrama de fases é análogo ao comportamento do modelo ANNNI usual de spin $\frac{1}{2}$, embora as sinuosidades das fronteiras de fases persistam para esse 


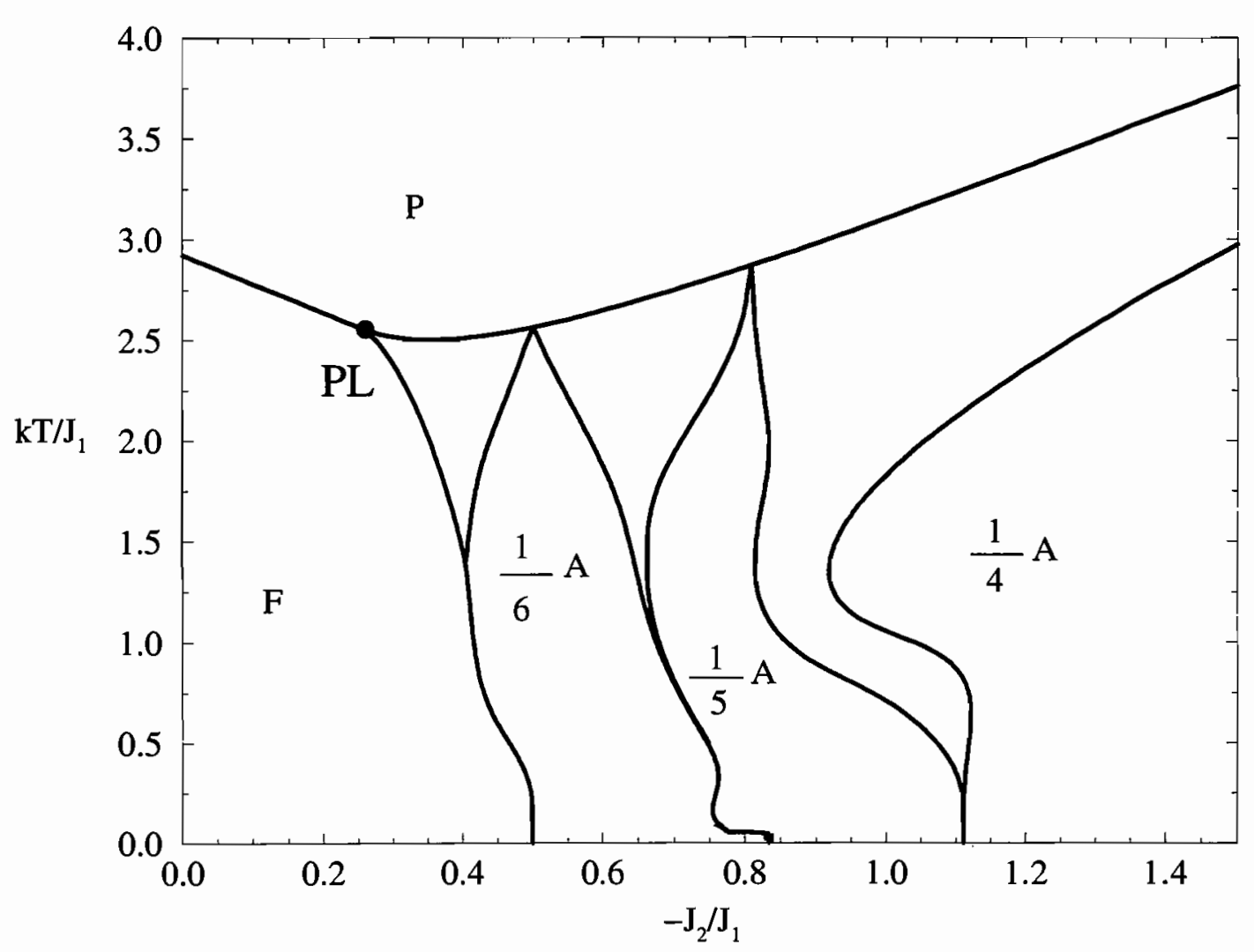

Figura 4.24: Diagrama de fases global do modelo no plano $k T / J_{1}$ versus $-J_{2} / J_{1}$ para $D_{0} / J_{1}=2.2$ e $\sigma / J_{1}=2.3$.

valor de $\sigma$. A outra região consiste predominantemente da fase $1 / 5 \mathrm{~A}$ e essa região é limitada à porção do diagrama de fases onde $1<-J_{2} / J_{1}<1.2 \mathrm{e}$ $0<k T / J_{1}<1$. Nessa região, as fases moduladas surgem do ponto de multifases $\left(-J_{2} / J_{1}=1.1778 \cdots, k T / J_{1}=0\right)$. Também localizamos um ponto triplo $(\mathrm{PT})$ em $\left(-J_{2} / J_{1}=0.977795 \cdots, k T / J_{1}=0.695 \cdots\right)$, no qual as fases $1 / 4 \mathrm{~A}, 1 / 5 \mathrm{~A}$ e $3 / 14 \mathrm{~A}$ se encontram. Conforme $\sigma / J_{1}$ aumenta, a região modulada menor ocupa porções ainda menores do diagrama de fases até desaparecer completamente. O comportamento do modelo no limite $\sigma / J_{1} \rightarrow \infty$ será discutido na próxima secção. 


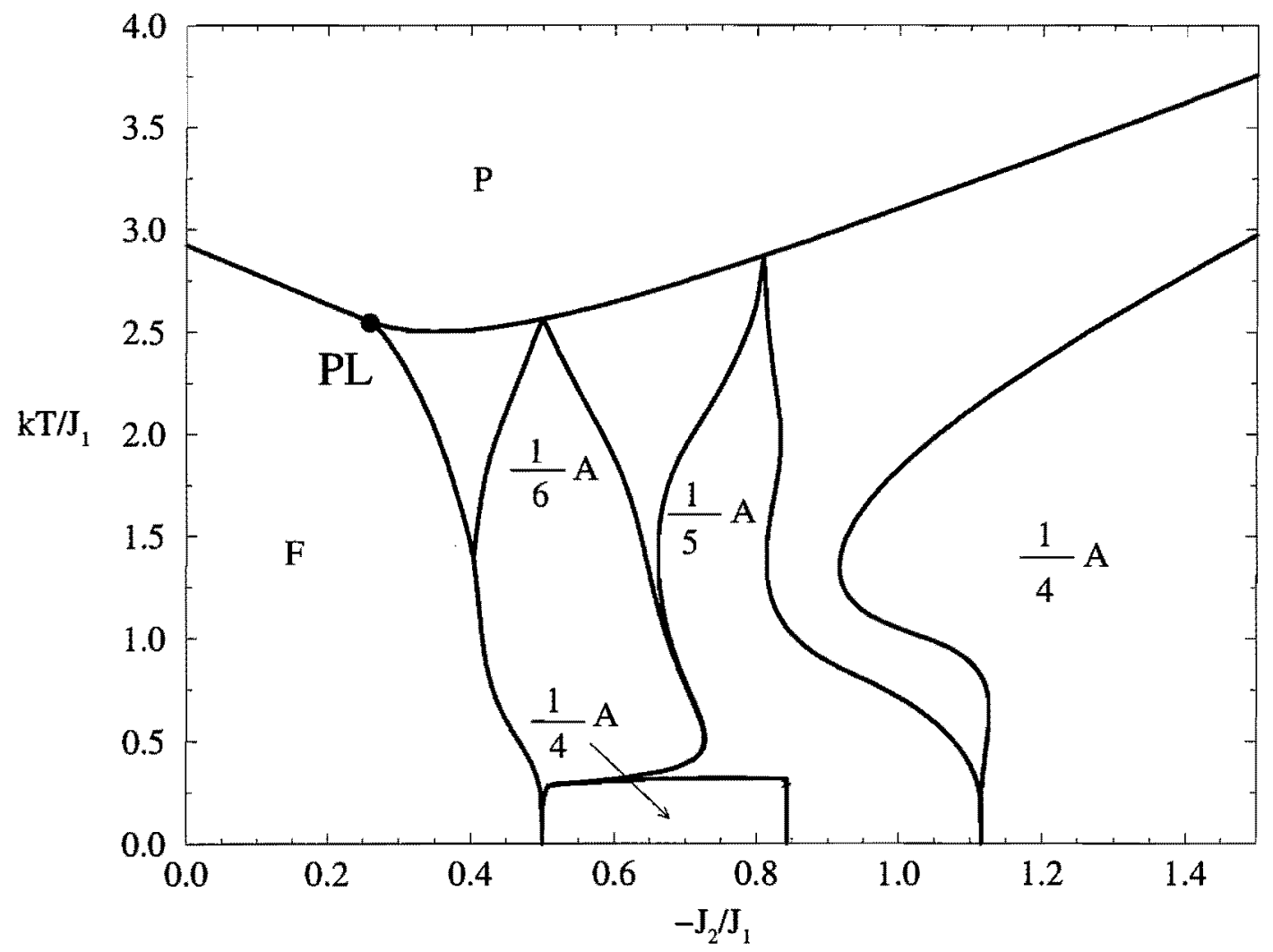

Figura 4.25: Diagrama de fases global do modelo no plano $k T / J_{1}$ versus $-J_{2} / J_{1}$ para $D_{0} / J_{1}=2.2$ e $\sigma / J_{1}=2.31$. 


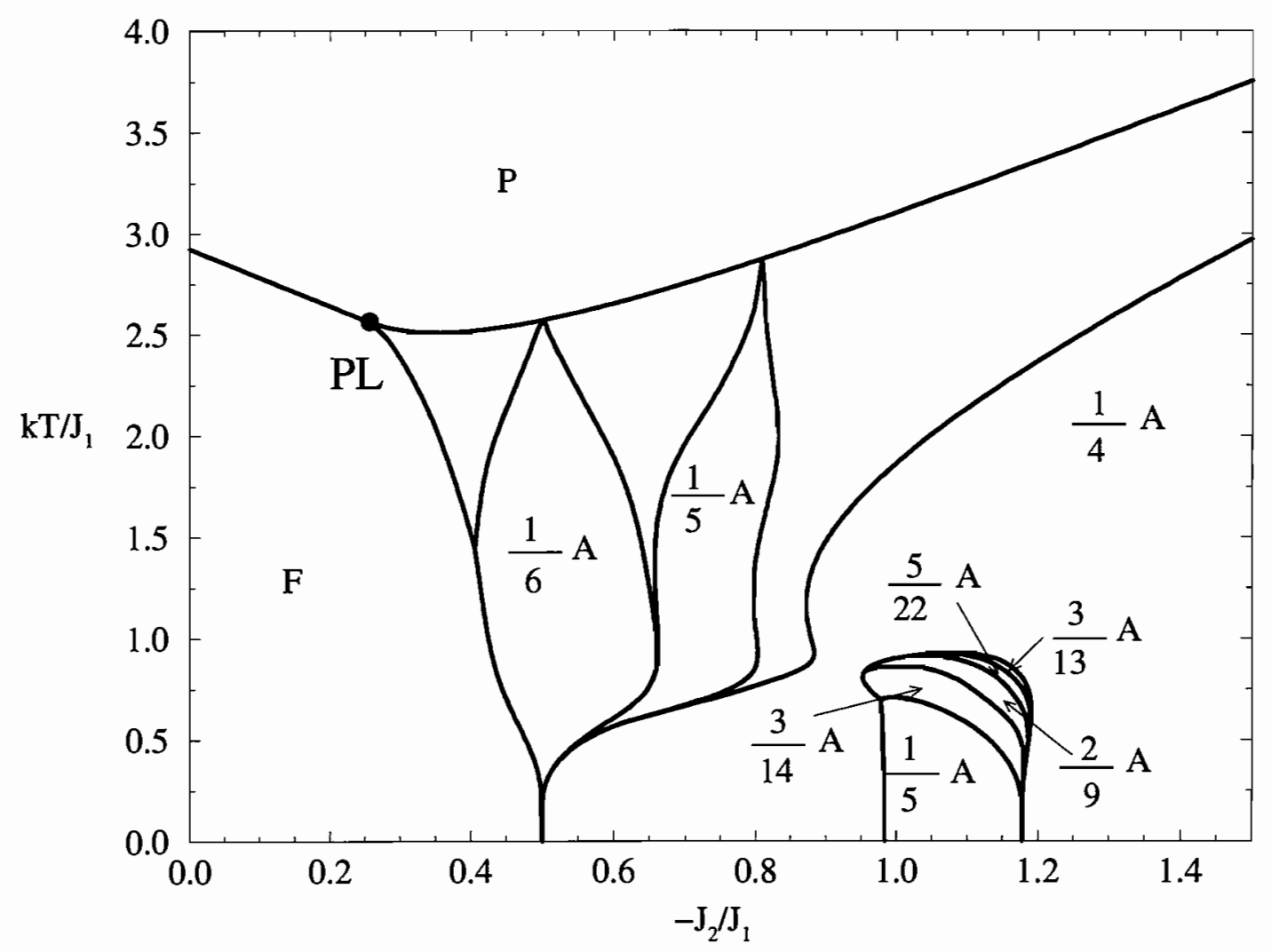

Figura 4.26: Diagrama de fases global do modelo no plano $k T / J_{1}$ versus $-J_{2} / J_{1}$ para $D_{0} / J_{1}=2.2$ e $\sigma / J_{1}=2.45$. 


\subsection{O modelo ANNNI com spin $1 / 2$ e diluição de sítios}

Nesta secção, ainda na aproximação de campo médio, mostramos que no limite $\sigma / J_{1} \rightarrow \infty$ o modelo ANNNI com spin 1 e campo cristalino deltabimodal corresponde ao modelo ANNNI usual com spin $\frac{1}{2}$ e diluição de sítios, o qual, por sua vez, pode ser mapeado ao modelo ANNNI usual com spin $\frac{1}{2}$. O modelo é definido numa rede cúbica simples, com variáveis de spin $S_{\mathbf{r}}= \pm 1$ nos sítios

$$
\mathbf{r}=x \hat{\mathbf{x}}+y \hat{\mathbf{y}}+z \hat{\mathbf{z}}
$$

sendo $x, y$ e $z$ inteiros entre 1 e $N$ (número de spins em cada uma das três direções) e $(\hat{\mathbf{x}}, \hat{\mathbf{y}}, \hat{\mathbf{z}})$ os vetores de base da célula primitiva. A hamiltoniana pode ser escrita

$$
\mathcal{H}=-\frac{1}{2} \sum_{\mathbf{r}, \mathbf{r}^{\prime}} J_{\mathbf{r r}} S_{\mathbf{r}} S_{\mathbf{r}^{\prime}} c_{\mathbf{r}} c_{\mathbf{r}^{\prime}}
$$

onde as somas em $\mathbf{r}$ e $\mathbf{r}^{\prime}$ são realizadas sobre todos os sítios da rede e as interações $J_{\mathbf{r r}^{\prime}}$ são dadas pela equação 1.3. As variáveis $c_{\mathbf{r}}$ são denominadas variáveis de ocupação, podendo assumir os valores 1 (sítio ocupado) ou 0 (sítio vazio) e obedecem a uma distribuição de probabilidades independente e identicamente distribuída para cada sítio

$$
P\left(c_{\mathbf{r}}\right)=p \delta\left(c_{\mathbf{r}}-1\right)+(1-p) \delta\left(c_{\mathbf{r}}\right)
$$

onde $p=1$ corresponde ao modelo puro (sem diluição). Como discutido na introdução desta tese, em geral podem ser considerados dois tipos de desordem. No caso da desordem temperada (quenched) as médias térmica e configuracional são independentes entre si, ao contrário do caso recozido (annealed). A desordem recozida em geral não é encontrada em sistemas magnéticos reais, 
uma vez que na maior parte dos casos a energia magnética não determina a desordem configuracional (Stinchcombe, 1983). Sendo assim, em nossos cálculos consideramos a desordem temperada.

A seguir vamos obter a energia livre $G$ usando a desigualdade de Bogoliubov (Falk, 1970; Callen, 1985). No caso temperado a energia livre $G$ é a média configuracional das energias livres $G\left(\left\{c_{\mathbf{r}}\right\}\right)$ para uma configuração fixa da desordem, ou seja

$$
G=\left\langle G\left(\left\{c_{\mathbf{r}}\right\}\right)\right\rangle_{c}
$$

onde

$$
\langle(\cdots)\rangle_{c}=\int_{-\infty}^{+\infty} \prod_{\mathbf{r}} \mathrm{d} c_{\mathbf{r}}(\cdots) P\left(c_{\mathbf{r}}\right),
$$

sendo $P\left(c_{\mathbf{r}}\right)$ a distribuição de probabilidades da equação (4.22). Tomando a média configuracional da desigualdade de Bogoliubov obtemos

$$
G \leq\left\langle G_{0}\left(\left\{c_{\mathbf{r}}\right\}\right)+\left\langle\mathcal{H}\left(\left\{c_{\mathbf{r}}\right\}\right)-\mathcal{H}_{0}\left(\left\{c_{\mathbf{r}}\right\}\right)\right\rangle_{0}\right\rangle_{c} \equiv \Phi
$$

onde a média $\langle\cdots\rangle_{0}$ é tomada em relação a uma hamiltoniana arbitrária $\mathcal{H}_{0}$. Como se trata de um cálculo variacional, escolhemos a hamiltoniana tentativa $\mathcal{H}_{0}$ de forma a levar em conta a maior quantidade de informação possível da hamiltoniana original, mas ainda permitindo resolver o lado direito da equação (4.25). Sendo assim, o parâmetro variacional será introduzido como um campo efetivo que depende do particular sítio r, ou seja,

$$
\mathcal{H}_{0}=-\sum_{\mathbf{r}} \eta_{\mathbf{r}} S_{\mathbf{r}} c_{\mathbf{r}}
$$

O termo $G_{0}$ na equação (4.25) é obtido a partir da hamiltoniana tentativa por meio da equação

$$
G_{0}=-\frac{1}{\beta} \ln Z_{0},
$$


sendo

$$
Z_{0}=\sum_{\{S\}}^{\prime} e^{\beta \sum_{\mathbf{r}}^{\prime} \eta_{\mathbf{r}} S_{\mathbf{r}}}=\Pi_{\mathbf{r}}^{\prime}\left[\sum_{S_{\mathbf{r}}= \pm 1} e^{\beta \eta_{\mathbf{r}} S_{\mathbf{r}}}\right]
$$

onde a restrição ' indica que as somas devem ser efetuadas apenas sobre os sítios não diluídos $\left(c_{\mathbf{r}}=1\right)$. Para escrevermos $Z_{0}$ em função das variáveis de ocupação $c_{\mathbf{r}}$, introduzimos o fator $\left(1+c_{\mathbf{r}}\right) / 2$ na equação $(4.28)$ e a produtória passa a ser sobre todos os sítios da rede, ou seja,

$$
Z_{0}=\prod_{\mathbf{r}}\left[\sum_{S_{\mathbf{r}}= \pm 1} e^{\beta \eta_{\mathbf{r}} S_{\mathbf{r}}} \frac{\left(1+c_{\mathbf{r}}\right)}{2}\right]
$$

Finalmente, efetuando a soma entre colchetes

$$
Z_{0}=\prod_{\mathbf{r}}\left[\cosh \left(\beta \eta_{\mathbf{r}} c_{\mathbf{r}}\right)\left(1+c_{\mathbf{r}}\right)\right]
$$

Substituindo a equação acima em (4.27) obtemos

$$
G_{0}=-\frac{1}{\beta} \ln \left[\cosh \left(\beta \eta_{\mathbf{r}} c_{\mathbf{r}}\right)\left(1+c_{\mathbf{r}}\right)\right] .
$$

Agora, a fim de obtermos a energia livre $G$, resta-nos calcular

$$
\left\langle\mathcal{H}-\mathcal{H}_{0}\right\rangle_{0}=-\frac{1}{2} \sum_{\mathbf{r r}^{\prime}}^{\prime} J_{\mathbf{r r}^{\prime}}\left\langle S_{\mathbf{r}} S_{\mathbf{r}^{\prime}}\right\rangle_{0}+\sum_{\mathbf{r}}^{\prime}\left\langle\eta_{\mathbf{r}} S_{\mathbf{r}}\right\rangle_{0}
$$

onde, como antes, o índice ' indica que as somas devem ser efetuadas apenas sobre os sítios não-diluídos. Para reescrevermos as somas de forma que sejam efetuadas sobre todos os sítios, fizemos a substituição $S_{\mathbf{r}} \rightarrow S_{\mathbf{r}} c_{\mathbf{r}}$ na equação (4.32) obtendo

$$
\left\langle\mathcal{H}-\mathcal{H}_{0}\right\rangle_{0}=-\frac{1}{2} \sum_{\mathbf{r} \mathbf{r}^{\prime}} J_{\mathbf{r r}}\left\langle S_{\mathbf{r}} S_{\mathbf{r}^{\prime}} c_{\mathbf{r}} c_{\mathbf{r}^{\prime}}\right\rangle_{0}+\sum_{\mathbf{r}}\left\langle\eta_{\mathbf{r}} S_{\mathbf{r}} c_{\mathbf{r}}\right\rangle_{0}
$$

com a soma sobre todos os sítios da rede. É fácil mostrar que

$$
\begin{aligned}
\left\langle S_{\mathbf{r}} S_{\mathbf{r}^{\prime}} c_{\mathbf{r}} c_{\mathbf{r}^{\prime}}\right\rangle_{0} & =c_{\mathbf{r}} c_{\mathbf{r}^{\prime}}\left\langle S_{\mathbf{r}}\right\rangle_{0}\left\langle S_{\mathbf{r}^{\prime}}\right\rangle_{0} \\
\left\langle\eta_{\mathbf{r}} S_{\mathbf{r}} c_{\mathbf{r}}\right\rangle_{0} & =\eta_{\mathbf{r}} c_{\mathbf{r}}\left\langle S_{\mathbf{r}}\right\rangle_{0}
\end{aligned}
$$


onde

$$
\left\langle S_{\mathbf{r}}\right\rangle_{0}=\tanh \left(\beta \eta_{\mathbf{r}}\right)
$$

Substituindo os resultados (4.31), (4.33), (4.34) e (4.35) no lado direito da desigualdade de Bogoliubov (4.25) obtemos

$$
\Phi=-\frac{1}{\beta} \sum_{\mathbf{r}}\left\langle\ln \left[\cosh \left(\beta \eta_{\mathbf{r}} c_{\mathbf{r}}\right)\right]\left(1+c_{\mathbf{r}}\right)\right\rangle_{c}-\frac{1}{2} \sum_{\mathbf{r}, \mathbf{r}^{\prime}} J_{\mathbf{r r}^{\prime}} m_{\mathbf{r}} m_{\mathbf{r}^{\prime}}+\sum_{\mathbf{r}} \eta_{\mathbf{r}} m_{\mathbf{r}}
$$

onde definimos

$$
m_{\mathbf{r}}=\left\langle c_{\mathbf{r}}\right\rangle_{c} \tanh \left(\beta \eta_{\mathbf{r}}\right)
$$

Sendo a desordem identicamente distribuída sobre todos os sítios da rede, não esperamos que a aleatoriedade modifique o tipo de ordenamento magnético. Por isso vamos impor que o parâmetro variacional dependa apenas da coordenada $z$, ou seja

$$
\eta_{\mathbf{r}} \equiv \eta_{z} \quad(z=\mathbf{r} \cdot \hat{\mathbf{z}})
$$

o que implica

$$
m_{\mathbf{r}} \equiv m_{z}
$$

O parâmetro variacional $\eta_{z}$ é calculado impondo-se que $\Phi$ seja estacionária ou seja,

$$
\frac{\partial \Phi}{\partial \eta_{z}}=0
$$

No ponto estacionário de $\Phi$ o parâmetro $m_{z}$ coincide com a magnetização por spin na camada $z$ e

$$
\begin{aligned}
\eta_{z} & =4 J_{0} m_{z}+J_{1}\left(m_{z+1}+m_{z-1}\right)+J_{2}\left(m_{z+2}+m_{z-2}\right) \\
m_{z} & =\left\langle c_{z}\right\rangle_{c} \tanh \left(\beta \eta_{z}\right) .
\end{aligned}
$$


Com as equações (4.42), (4.43) e calculando a média configuracional, o funcional da energia livre fica

$$
\begin{aligned}
N^{-3} \Phi\left(T, N ; \eta_{z}\right)= & -p k T \ln 2-\frac{p k T}{N} \sum_{z} \ln \left[\cosh \left(\beta \eta_{z}\right)\right] \\
& -\frac{1}{2 N} \sum_{z}\left[4 J_{0} m_{z}^{2}+J_{1} m_{z}\left(m_{z+1}+m_{z-1}\right)\right. \\
& \left.+J_{2} m_{z}\left(m_{z+2}+m_{z-2}\right)\right]+\frac{1}{N} \sum_{z} \eta_{z} m_{z}
\end{aligned}
$$

e a magnetização por spin na camada $z$ é obtida a partir das equações de campo médio

$$
m_{z}=p \tanh \left(\beta \eta_{z}\right)
$$

e

$$
\eta_{z}=4 J_{0} m_{z}+J_{1}\left(m_{z+1}+m_{z-1}\right)+J_{2}\left(m_{z+2}+m_{z-2}\right) .
$$

Para $p=1$ o funcional da energia livre (4.44) reduz-se ao funcional da energia livre do modelo puro, diferindo apenas por um fator multiplicativo para $p \neq 1$. Concluímos desse fato que, na aproximação de campo médio, o diagrama de fases do modelo ANNNI diluído exibe o mesmo comportamento do diagrama do modelo puro para qualquer valor de $p$ e pode ser obtido a partir do modelo puro pelo mapeamento $k T / J_{1} \rightarrow p k T / J_{1}$. Para ilustrar o que acabamos de dizer, mostramos na figura 4.27 as linhas críticas obtidas para diferentes valores de $p$. Para obter a expressão das linhas críticas, efetuamos a expansão de Landau do modelo. Os cálculos procedem de maneira análoga à desenvolvida no Capítulo 3 , secção 3.4, e por esse motivo apresentamos aqui apenas o resultado obtido para as linhas críticas

$$
k T_{c}=p J\left(q_{c}\right),
$$

sendo $J\left(q_{c}\right)$ dado pela equação (3.52). 


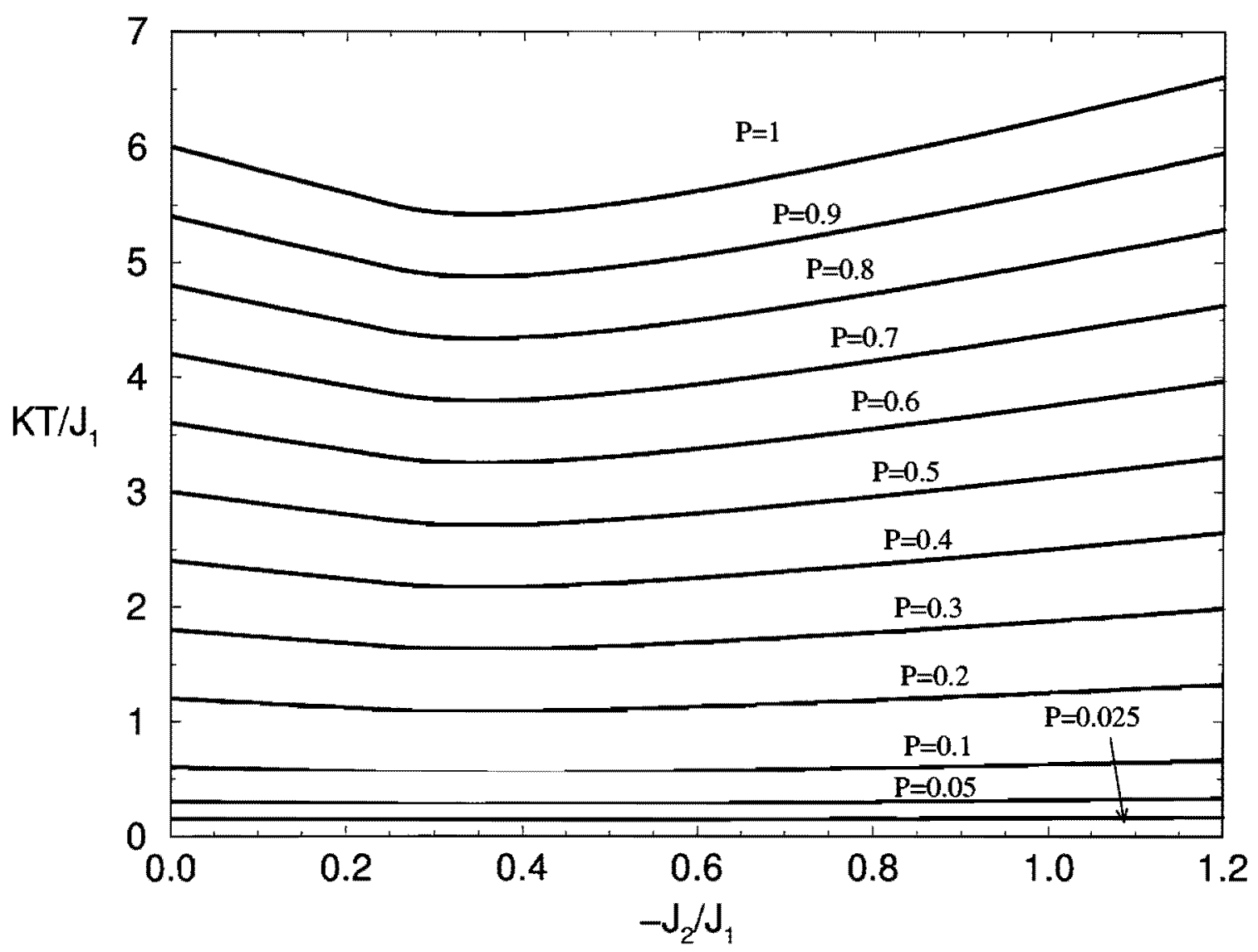

Figura 4.27: Linhas críticas do modelo ANNNI diluído para vários valores de $p$. O caso $p=1$ corresponde ao modelo puro, ao passo que para $p=0$ o modelo é completamente diluído. As linhas referentes a $p \neq 1$ podem ser obtidas a partir da linha referente ao modelo puro pelo mapeamento $k T / J_{1} \rightarrow p k T / J_{1}$.

Ainda na aproximação de campo médio, a seguir mostramos que o modelo ANNNI usual com spin $\frac{1}{2}$ e diluição de sítios é exatamente o limite $\sigma / J_{1} \rightarrow \infty$ do modelo ANNNI com spin 1 e campo cristalino delta-bimodal. Para isso, generalizamos a distribuição de probabilidades (4.1) do campo cristalino na forma

$$
\left.P(D)=p \delta\left(D-D_{0}+\sigma\right)+(1-p) \delta\left(D-D_{0}-\sigma\right)\right]
$$

Seguindo os cálculos de campo médio desenvolvidos na secção 3.2 , obtivemos 
o funcional da energia livre

$$
\begin{aligned}
N^{-3} \Phi= & -k T \sum_{z}\left\{p \ln \left[1+2 e^{-\frac{\left(D_{0}-\sigma\right)}{k T}} \cosh \frac{\eta_{z}}{k T}\right]\right. \\
+ & \left.(1-p) \ln \left[1+2 e^{-\frac{\left(D_{0}+\sigma\right)}{k T}} \cosh \frac{\eta_{z}}{k T}\right]\right\} \\
& -\frac{1}{2 N} \sum_{z}\left[4 J_{1} m_{z}^{2}+J_{1} m_{z}\left(m_{z+1}+m_{z-1}\right)\right. \\
& \left.+J_{2} m_{z}\left(m_{z+2}+m_{z-2}\right)\right]+\frac{1}{N} \sum_{z} \eta_{z} m_{z}
\end{aligned}
$$

e a equação de campo médio

$$
m_{z}=\frac{2 p e^{-\frac{\left(D_{0}-\sigma\right)}{k T}} \sinh \frac{\eta_{z}}{k T}}{1+2 e^{-\frac{\left(D_{0}-\sigma\right)}{k T}} \cosh \frac{\eta_{z}}{k T}}+\frac{2(1-p) e^{\left.-\frac{\left(D_{0}+\sigma\right.}{k T}\right)} \sinh \frac{\eta_{z}}{k T}}{1+2 e^{\left.-\frac{\left(D_{0}+\sigma\right.}{k T}\right)} \cosh \frac{\eta_{z}}{k T}}
$$

onde $\eta_{z}$ é dado pela equação (4.46). No limite $\sigma / J_{1} \rightarrow \infty$, a equação (4.49) fica

$$
\begin{aligned}
N^{-3} \Phi= & -p k T \ln 2+p\left(D_{0}-\sigma\right)-\frac{p k T}{N} \sum_{z} \ln \left[\cosh \frac{\eta_{z}}{k T}\right] \\
& -\frac{1}{2 N} \sum_{z}\left[4 J_{1} m_{z}^{2}+J_{1} m_{z}\left(m_{z+1}+m_{z-1}\right)\right. \\
& \left.+J_{2} m_{z}\left(m_{z+2}+m_{z-2}\right)\right]+\frac{1}{N} \sum_{z} \eta_{z} m_{z}
\end{aligned}
$$

a qual difere do funcional da energia livre (4.44) apenas pela constante aditiva $p\left(D_{0}-\sigma\right)$. Além disso, para $\sigma / J_{1} \rightarrow \infty$, a magnetização por spin por camada (4.50) torna-se exatamente a equação (4.45), que foi obtida para o modelo ANNNI usual com spin $\frac{1}{2}$ e diluição de sítios. Assim, mostramos que o limite $\sigma / J_{1} \rightarrow \infty$ do modelo ANNNI com spin 1 e termo de campo cristalino delta-bimodal corresponde ao modelo ANNNI usual com spin $\frac{1}{2}$ e diluição de sítios. Vale reforçar que todos os resultados obtidos nesta secção são válidos na aproximação de campo médio. 


\section{Capítulo 5}

\section{Resultados para uma distribuição gaussiana}

Neste capítulo apresentamos os resultados obtidos para uma distribuição gaussiana do campo cristalino

$$
P(D)=\frac{1}{\sigma \sqrt{2 \pi}} \exp \left[-\frac{\left(D-D_{0}\right)^{2}}{2 \sigma^{2}}\right],
$$

onde o valor médio $D_{0}$ e o desvio padrão $\sigma$ representam, respectivamente, a intensidade e a desordem do campo cristalino. No caso em que $\sigma=0$ recuperamos o modelo ANNNI com spin 1 e campo cristalino constante (Cadorin e Yokoi, 1998). Considerando $\sigma \neq 0$, na secção 5.1 construímos os diagramas de fases do modelo em $T=0$. Na seç̧ão 5.2 estudamos a região de altas temperaturas do modelo, correspondente às linhas críticas e pontos tricríticos. Finalmente, na seção 5.3 obtivemos os diagramas de fases do modelo para $T \neq 0$. 


\subsection{Diagramas de fases em $T=0$}

Com a distribuição de probabilidades (5.1), a equação de campo médio (3.29) fica

$$
m_{z}=\frac{\operatorname{sgn}\left(\eta_{z}\right)}{2} \operatorname{erfc}\left(\frac{\left(D_{0}-\left|\eta_{z}\right|\right)}{2 \sigma}\right),
$$

onde $\eta_{z}$ é dado pela equação $3.30 \mathrm{e}$

$$
\operatorname{erfc}(x)=\frac{2}{\sqrt{\pi}} \int_{x}^{\infty} e^{-t^{2}} \mathrm{~d} t
$$

é a função erro complementar. Ainda precisamos obter o funcional da energia livre em $T=0$. Para isso, observamos inicialmente que o primeiro termo no lado direito da equação (3.27) é o único que depende explicitamente da distribuição de probabilidades considerada. Como esse termo não depende de $m_{z}$ podemos ignorá-lo ao compararmos as energias livres das diferentes soluções. Portanto, para o funcional da energia livre em $T=0$ vamos levar em conta apenas os termos que dependem de $m_{z}$,

$$
\begin{aligned}
N^{-3} \Phi= & -\frac{1}{N} \sum_{z} \int_{0}^{\eta_{z}} m_{z} d \eta_{z}+\frac{1}{2 N} \sum_{z}\left[4 J_{0} m_{z}^{2}+J_{1} m_{z}\left(m_{z+1}+m_{z-1}\right)\right. \\
& \left.+J_{2} m_{z}\left(m_{z+2}+m_{z-2}\right)\right] .
\end{aligned}
$$

Com as equações (5.2), (3.30) e (5.4) podemos construir os diagramas de fases do modelo em $T=0$.

Todos os diagramas de fases apresentados a seguir foram obtidos para $J_{0}=J_{1}$. As fronteiras entre as fases moduladas foram obtidas segundo o processo de ramificação por combinação de estruturas (Duxbury e Selke, 1983; Selke e Duxbury, 1984) descrito na secção 4.3. Para estudarmos os efeitos da desordem, construímos diagramas de fases do modelo no plano $\sigma / J_{1}$ versus 
- $J_{2} / J_{1}$ para um valor médio $D_{0} / J_{1}$ fixo. Para sermos sistemáticos nesse estudo, analogamente ao que fizemos no capítulo 4 , consideramos as 4 regiões definidas no diagrama de fases da figura 4.4. Em cada uma dessas quatro regiões fixamos diferentes valores de $D_{0} / J_{1}$, para os quais levantamos os diagramas de fases do modelo em $T=0$ no plano $\sigma / J_{1}$ versus $-J_{2} / J_{1}$. A notação empregada para as fases moduladas é a mesma empregada no capítulo 4 . Note que no caso da distribuição gaussiana do campo cristalino, a magnetização $m_{z}$ pode variar continuamente mesmo em $T=0$, não sendo necessário o uso da extensão $\left({ }^{\prime}\right)$.

$\mathrm{Na}$ região I construímos os diagramas de fases das figuras 5.1 e 5.2 para os valores médios $D_{0} / J_{1}=1$ e $D_{0} / J_{1}=2$, respectivamente. Na figura 5.1 observamos o surgimento das fases moduladas segundo o processo de ramificação por combinação de estruturas (Selke e Duxbury, 1984), explicado no capítulo anterior. As fases $\frac{1}{6} \mathrm{~A}$ e $\frac{1}{5} \mathrm{~A}$ são as mais proeminentes do diagrama de fases. Além disso, as fronteiras entre as fases não são retas, como no caso de uma distribuição bimodal. Não observamos a existência de fases tipo "B" (parcialmente desordenadas). Na figura 5.2 observamos um pequeno alargamento da região ocupada pelas fases moduladas, em comparação à figura 5.1, além do surgimento da fase $\frac{1}{8} \mathrm{~A}$. 


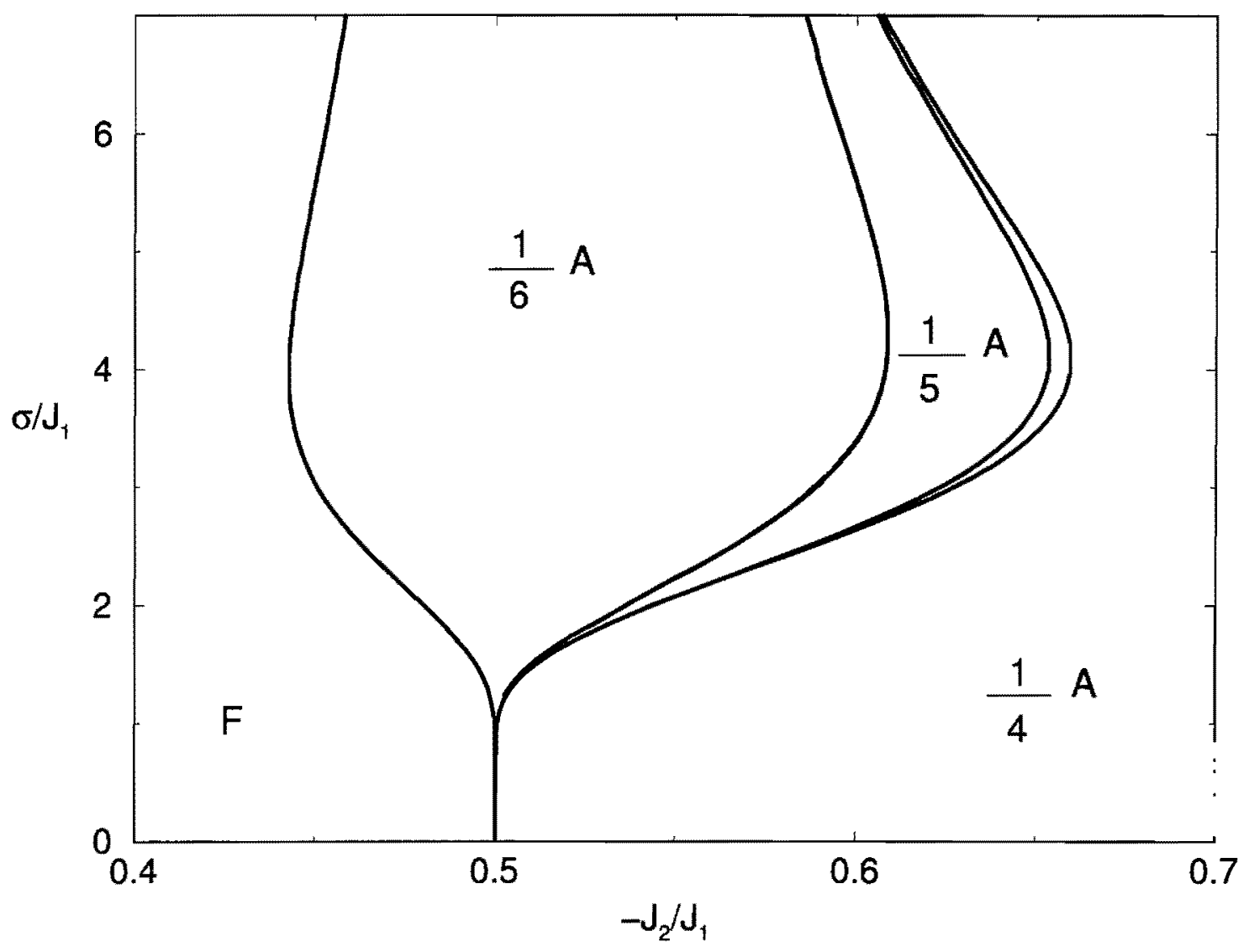

Figura 5.1: Diagrama de fases em $T=0$ no plano $\sigma / J_{1}$ versus $-J_{2} / J_{1}$ para $D_{0} / J_{1}=1$. 


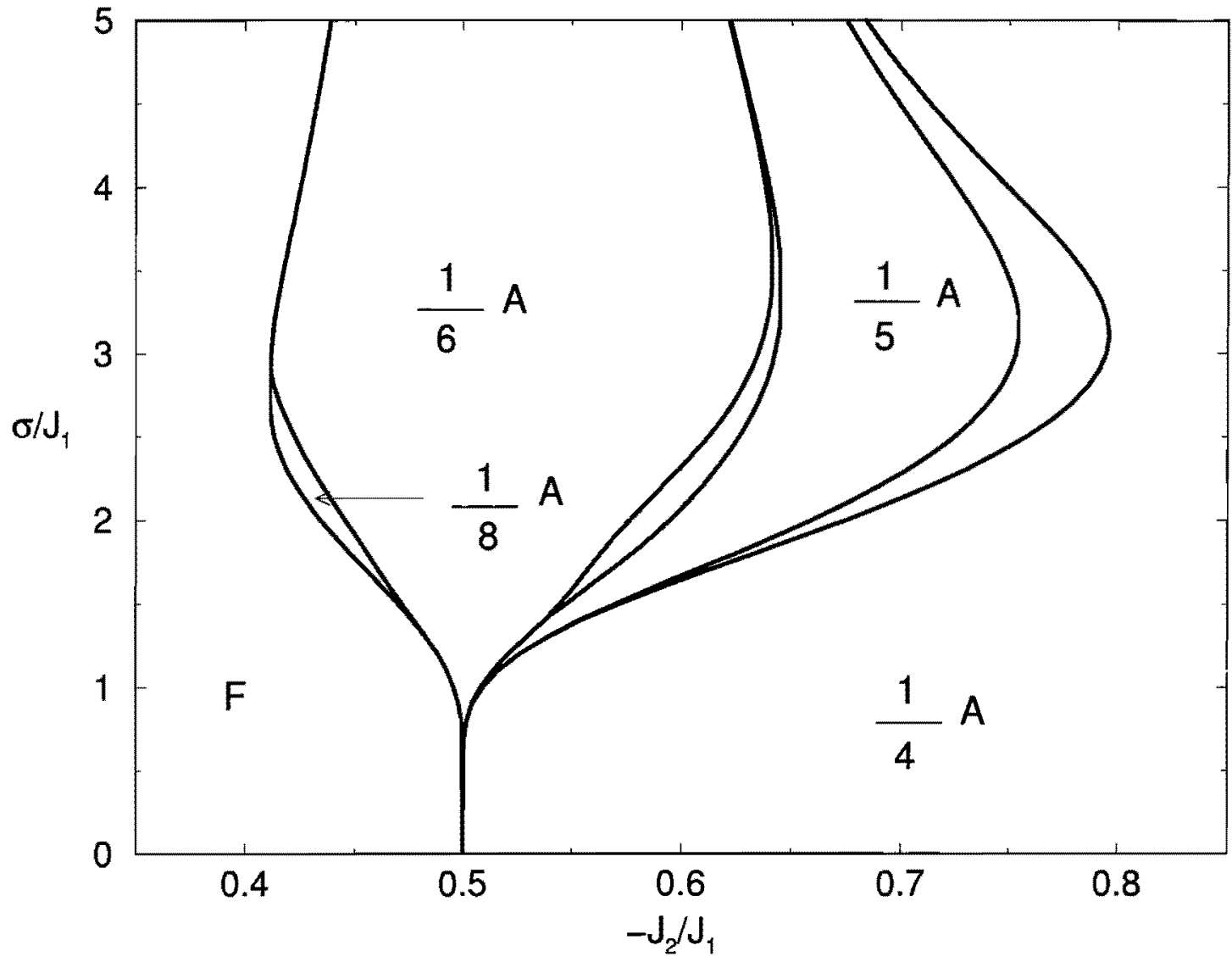

Figura 5.2: Diagrama de fases em $T=0$ no plano $\sigma / J_{1}$ versus $-J_{2} / J_{1}$ para $D_{0} / J_{1}=2$. 
$\mathrm{Na}$ região II consideramos os valores médios $D_{0} / J_{1}=2.2$ e $D_{0} / J_{1}=$ $2.666 \cdots$. Os diagramas de fases respectivos estão mostrados nas figuras 5.3 e 5.4. Nesses diagramas observamos que a fase $\frac{1}{6}$ é do tipo B até $\sigma / J_{1} \approx 2$. Aumentando-se a desordem, a fase $\frac{1}{6}$ sofre uma transição para o tipo A. Notamos também a existência da fase $\frac{1}{5} \mathrm{~B}$.

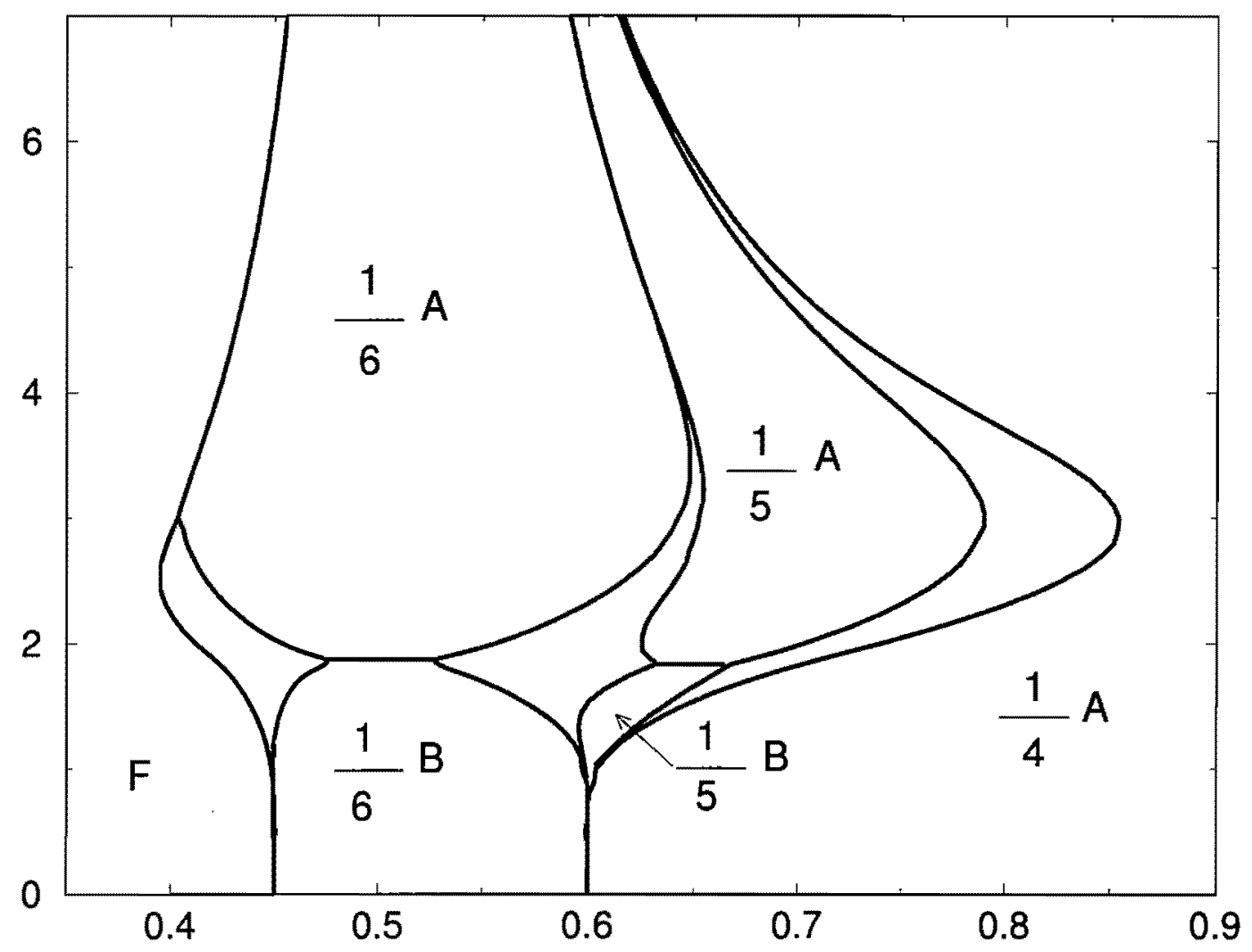

Figura 5.3: Diagrama de fases em $T=0$ no plano $\sigma / J_{1}$ versus $-J_{2} / J_{1}$ para $D_{0} / J_{1}=2.2$. 


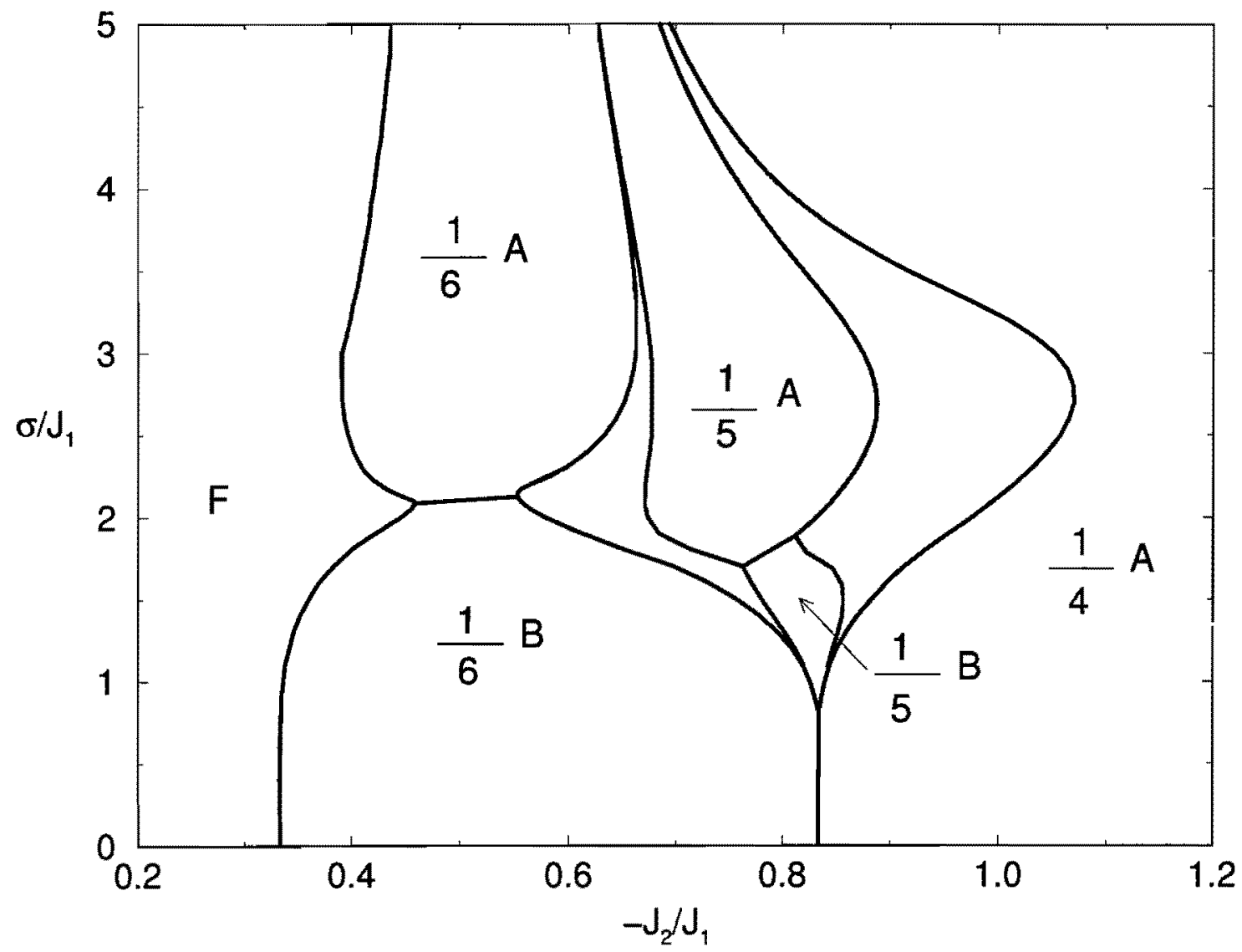

Figura 5.4: Diagrama de fases em $T=0$ no plano $\sigma / J_{1}$ versus $-J_{2} / J_{1}$ para $D_{0} / J_{1}=$ $2.666 \cdots$ 
$\mathrm{Na}$ região III construímos o diagrama de fases da figura 5.5 para o valor médio $D_{0} / J_{1}=2.8$. Nesse diagrama notamos a existência de duas novas fases: $\mathrm{F}^{\prime \prime}$ e $\frac{1}{4} \mathrm{~A}^{\prime \prime}$. A extensão (") indica que uma fase se torna paramagnética para $\sigma / J_{1}=0$ e $T=0$. As fases $F$ e $F^{\prime \prime}$ são separadas por uma transição de primeira ordem e tornam-se iguais no ponto crítico de coordenadas $\left(-J_{2} / J_{1}=\right.$ $\left.0.2 ; \sigma / J_{1}=2.2340768 \cdots\right)$. No apêndice B desenvolvemos explicitamente os cálculos para a obtenção das coordenadas do ponto crítico

$$
\begin{aligned}
-\frac{J_{2}}{J_{1}} & =3-\frac{D_{0}}{J_{1}} \\
\frac{\sigma}{J_{1}} & =\frac{D_{0}}{J_{1}} \sqrt{\frac{2}{\pi}}
\end{aligned}
$$




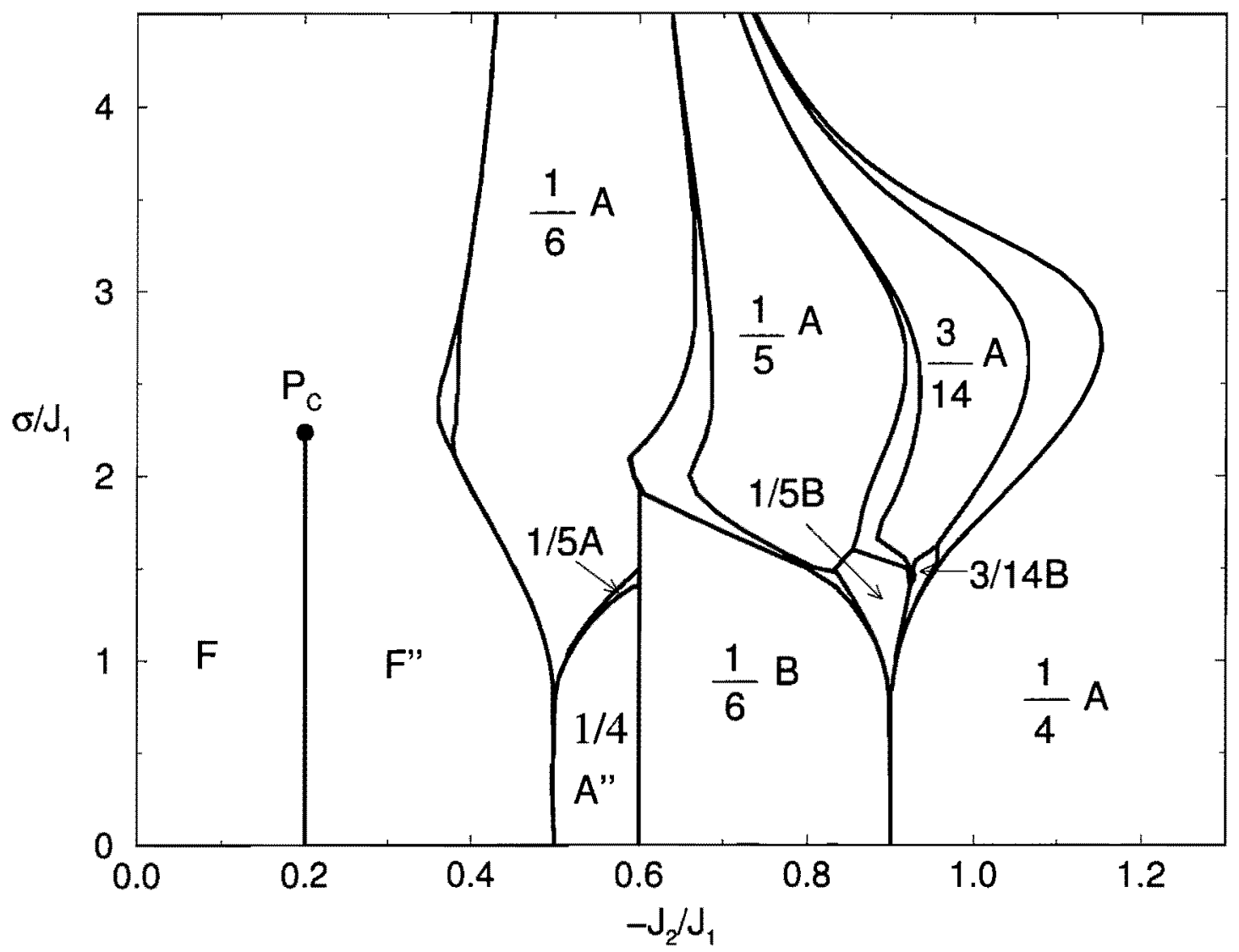

Figura 5.5: Diagrama de fases em $T=0$ no plano $\sigma / J_{1}$ versus $-J_{2} / J_{1}$ para $D_{0} / J_{1}=2.8$. As coordenadas do ponto crítico $\mathrm{P}_{\mathrm{c}}$ são $\left(-J_{2} / J_{1}=0.2 ; \sigma / J_{1}=2.2340768\right)$. 
Finalmente, na região IV consideramos os valores médios $D_{0} / J_{1}=3 \mathrm{e}$ $D_{0} / J_{1}=3.25$, para os quais os diagramas de fases estão apresentados nas figuras 5.6 e 5.7, respectivamente. No diagrama da figura 5.6 observamos que a fase $\frac{1}{6} \mathrm{~B}$ está ausente. Além disso, a porção estreita da fase $\frac{1}{5} \mathrm{~A}$ entre as fases $\frac{1}{4} \mathrm{~A}^{\prime \prime}$ e $\frac{1}{6} \mathrm{~A}$ unificou-se à sua porção mais larga. Entre as fases $\frac{1}{4} \mathrm{~A}^{\prime \prime}$ e $\frac{1}{5} \mathrm{~A}$ existe uma porção estreita da fase $\frac{3}{14} \mathrm{~A}$. Notamos que existe uma transição direta entre as fases $\frac{1}{4} \mathrm{~A}^{\prime \prime} \mathrm{e} \frac{1}{4} \mathrm{~A}$ para $\sigma / J_{1}=0$. O ponto crítico $\mathrm{P}_{C}$ está localizado em $\left(-J_{2} / J_{1}=0 ; \sigma / J_{1}=2.3936537 \cdots\right)$.

No diagrama da figura 5.7 observamos que a fase $\frac{1}{5} \mathrm{~B}$ está ausente. A porção estreita da fase $\frac{3}{14} \mathrm{~A}$ unificou-se à sua porção mais larga. Entre as fases $\frac{1}{4} \mathrm{~A}^{\prime \prime} \mathrm{e}$ $\frac{3}{14} \mathrm{~A}$ existe uma porção estreita da fase $\frac{4}{18} \mathrm{~A}$. Existe uma transição direta entre as fases $\frac{1}{4} \mathrm{~A}^{\prime \prime}$ e $\frac{1}{4} \mathrm{~A}$ para $\sigma / J_{1}>0$.

Em todos os diagramas de fases mostrados nas figuras 5.1 a 5.7 , para $\sigma / J_{1} \rightarrow \infty$, todas as linhas de transição colapsam sobre a reta $-J_{2} / J_{1}=0.5$, permanecendo no diagrama de fases em $T=0$ apenas as fases $\mathrm{F}$ e $\frac{1}{4} \mathrm{~A}$. 


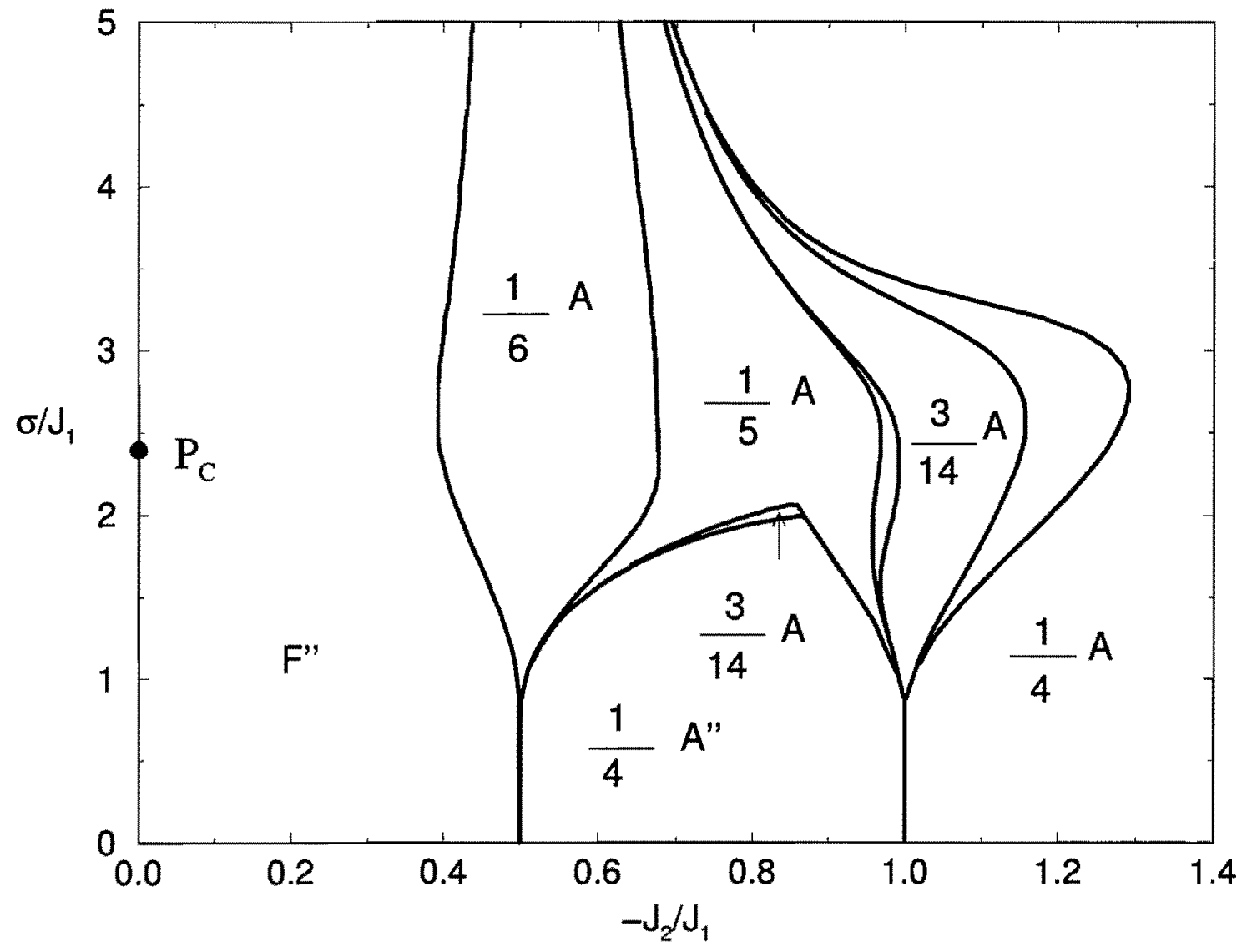

Figura 5.6: Diagrama de fases em $T=0$ no plano $\sigma / J_{1}$ versus $-J_{2} / J_{1}$ para $D_{0} / J_{1}=3$. As coordenadas do ponto crítico $\mathrm{P}_{\mathrm{C}}$ são $\left(-J_{2} / J_{1}=0 ; \sigma / J_{1}=2.3936537 \cdots\right)$. 


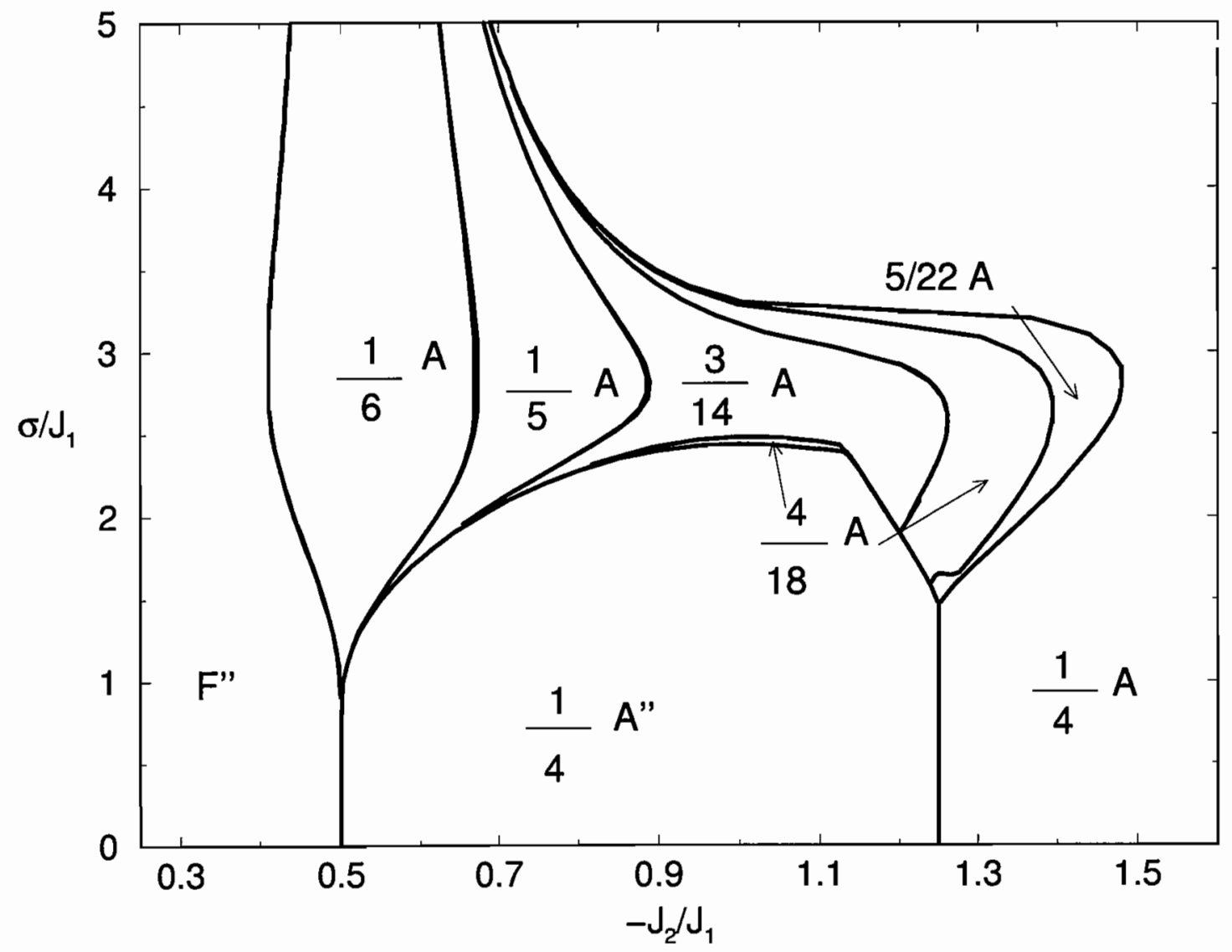

Figura 5.7: Diagrama de fases em $T=0$ no plano $\sigma / J_{1}$ versus $-J_{2} / J_{1}$ para $D_{0} / J_{1}=3.25$. 


\subsection{Linhas críticas e pontos tricríticos}

Para a distribuição de probabilidades gaussiana (5.1), a equação (3.53) da linha crítica fica

$$
k T_{c}=J\left(q_{c}\right) \int_{-\infty}^{+\infty} f(D) \exp \left[-\frac{\left(D-D_{0}\right)^{2}}{2 \sigma^{2}}\right] d D,
$$

e a condição (3.57) de estabilidade da linha

$$
\int_{-\infty}^{+\infty} f(D)[1-3 f(D)] \exp \left[-\frac{\left(D-D_{0}\right)^{2}}{2 \sigma^{2}}\right] d D<0,
$$

sendo

$$
f(D)=\frac{1}{1+\frac{1}{2} e^{\frac{D}{k T_{c}}}} .
$$

A quantidade $J\left(q_{c}\right)$ na equação (5.7) é dada pela equação (3.52). Com a distribuição gaussiana (5.1), a equação (3.59) fica

$$
\int_{-\infty}^{+\infty} f(D)[1-3 f(D)] \exp \left[-\frac{\left(D-D_{0}\right)^{2}}{2 \sigma^{2}}\right] d D=0,
$$

e o ponto tricrítico é então determinado pelas equações (5.7) e (5.10). A condição de estabilidade (3.61) desses pontos fica

$$
\int_{-\infty}^{+\infty}[f(D)]^{2}\left[1-\frac{5}{2} f(D)\right] \exp \left[-\frac{\left(D-D_{0}\right)^{2}}{2 \sigma^{2}}\right] d D>0 .
$$

Para verificarmos a influência da desordem sobre as linhas críticas e os pontos tricríticos do modelo, construímos linhas críticas para alguns valores de $D_{0}$ e $\sigma$. Para verificarmos como a desordem afeta o comportamento das linhas críticas, nas figuras (5.8) e (5.9) construímos linhas críticas obtidas para $\sigma / J_{1}=0.5$ e 1.5 , respectivamente. 


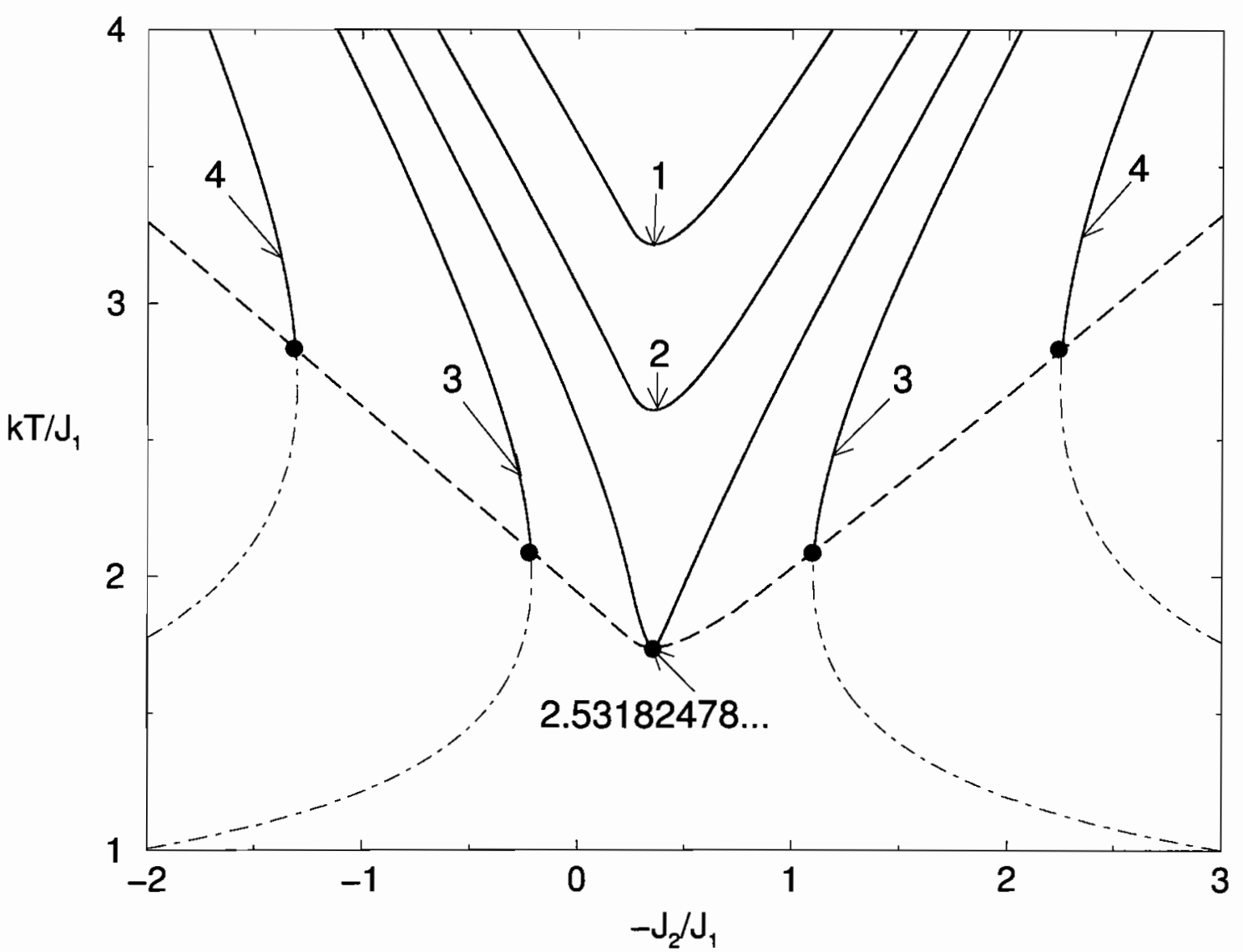

Figura 5.8: Linhas críticas no plano $k T / J_{1}$ versus $-J_{2} / J_{1}$ com $\sigma / J_{1}=0.5$ e alguns valores de $D_{0} / J_{1}$, representados pelos números associados a cada linha. Para esse valor de $\sigma / J_{1}$ o comportamento das linhas críticas é semelhante ao observado no caso $\sigma=0$ (figura 4.17). As linhas contínuas correspondem aos trechos das linhas críticas onde a condição de estabilidade (5.8) é satisfeita, ao passo que os trechos instáveis correspondem às linhas traço-ponto. Para esse valor de $\sigma$ os pontos tricríticos existem apenas para $D_{0} / J_{1} \geq$ $2.53182478 \cdots$ e estão representados pelos círculos cheios. As linhas tracejadas são os loci dos pontos tricríticos. 


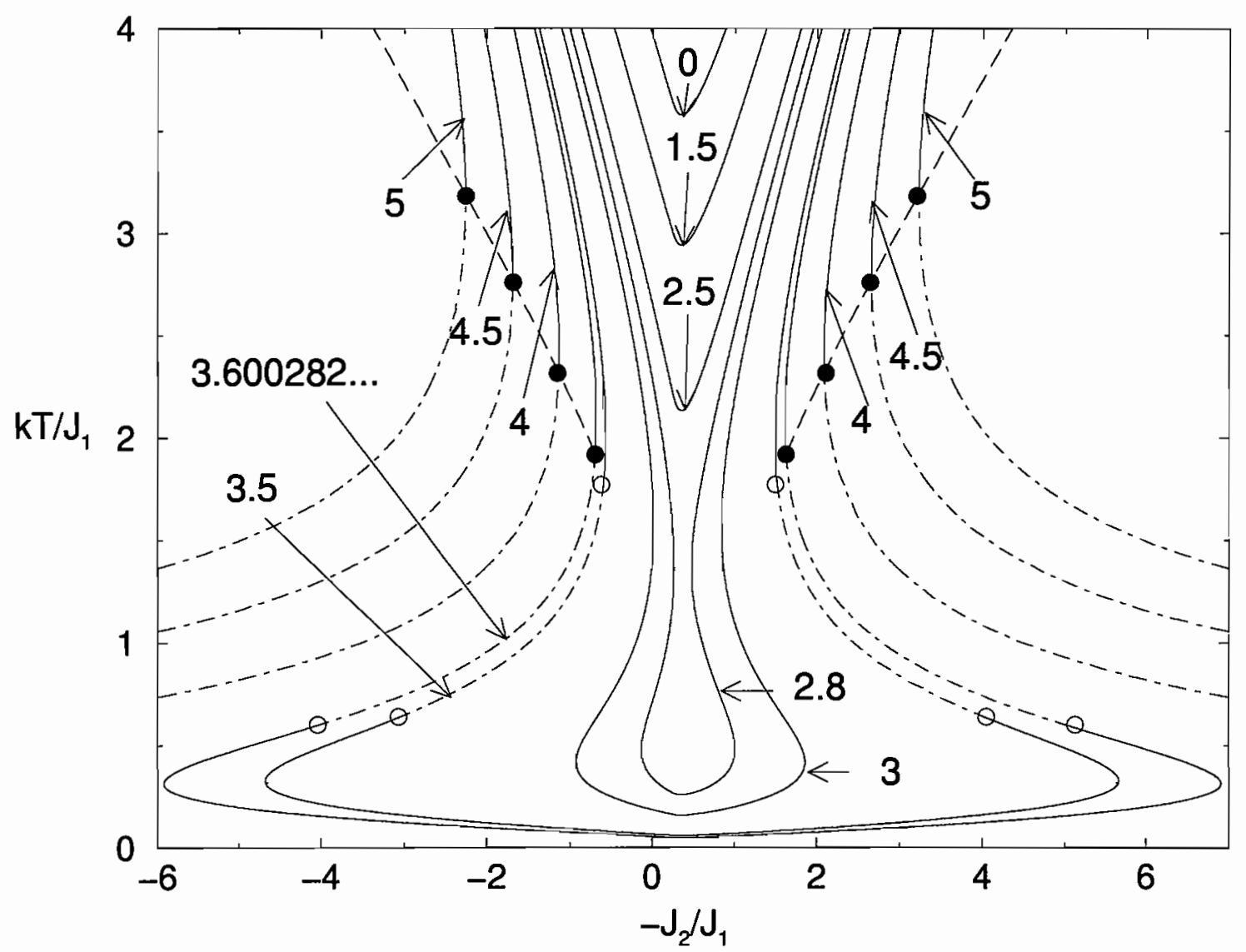

Figura 5.9: Linhas críticas no plano $k T / J_{1}$ versus $-J_{2} / J_{1}$ com $\sigma / J_{1}=1.5$ e alguns valores de $D_{0} / J_{1}$, representados pelos números associados a cada linha. Para esse valor de $\sigma$ o comportamento das linhas críticas é bastante distinto do observado no caso $\sigma=0$ (figura 4.17). Os pontos tricríticos existem apenas para $D_{0} / J_{1} \geq 3.600282 \cdots$. Os trechos das linhas críticas satisfazendo a condição de estabilidade (5.8) são representados pelas linhas contínuas, ao passo que as linhas traço-ponto representam os trechos onde as linhas críticas são instáveis. Os círculos vazios representam pontos tricríticos instáveis, ou seja, nesses pontos as equações (5.7) e (5.10) são satisfeitas, mas a condição de estabilidade dos pontos tricríticos (5.11) é violada. Os círculos cheios representam pontos tricríticos estáveis, onde as equações (5.7), (5.10) e (5.11) são satisfeitas. As linhas críticas para $D_{0} / J_{1}=4,4.5$ e 5 também possuem pontos tricríticos instáveis, embora não tenham sido incluídos na figura por estarem numa região fora da escala dos gráficos. As linhas tracejadas são os loci dos pontos tricríticos.

\section{INSTITUTO DE FÍSICA}

Serviço de Biblioteca e Infonrmaçăo

Tombo. $\frac{2587}{e x \cdot 1}$ 
Finalmente, na figura 5.10 mostramos a curva que delimita as regiões no plano $\sigma / J_{1}$ versus $D_{0} / J_{1}$ para as quais existem ou não pontos tricríticos. Concluímos que a desordem inibe a ocorrência de pontos tricríticos, ou seja, por maior que seja o valor de $D_{0}$, sempre haverá um valor de $\sigma$ a partir do qual os pontos tricríticos deixam de existir.

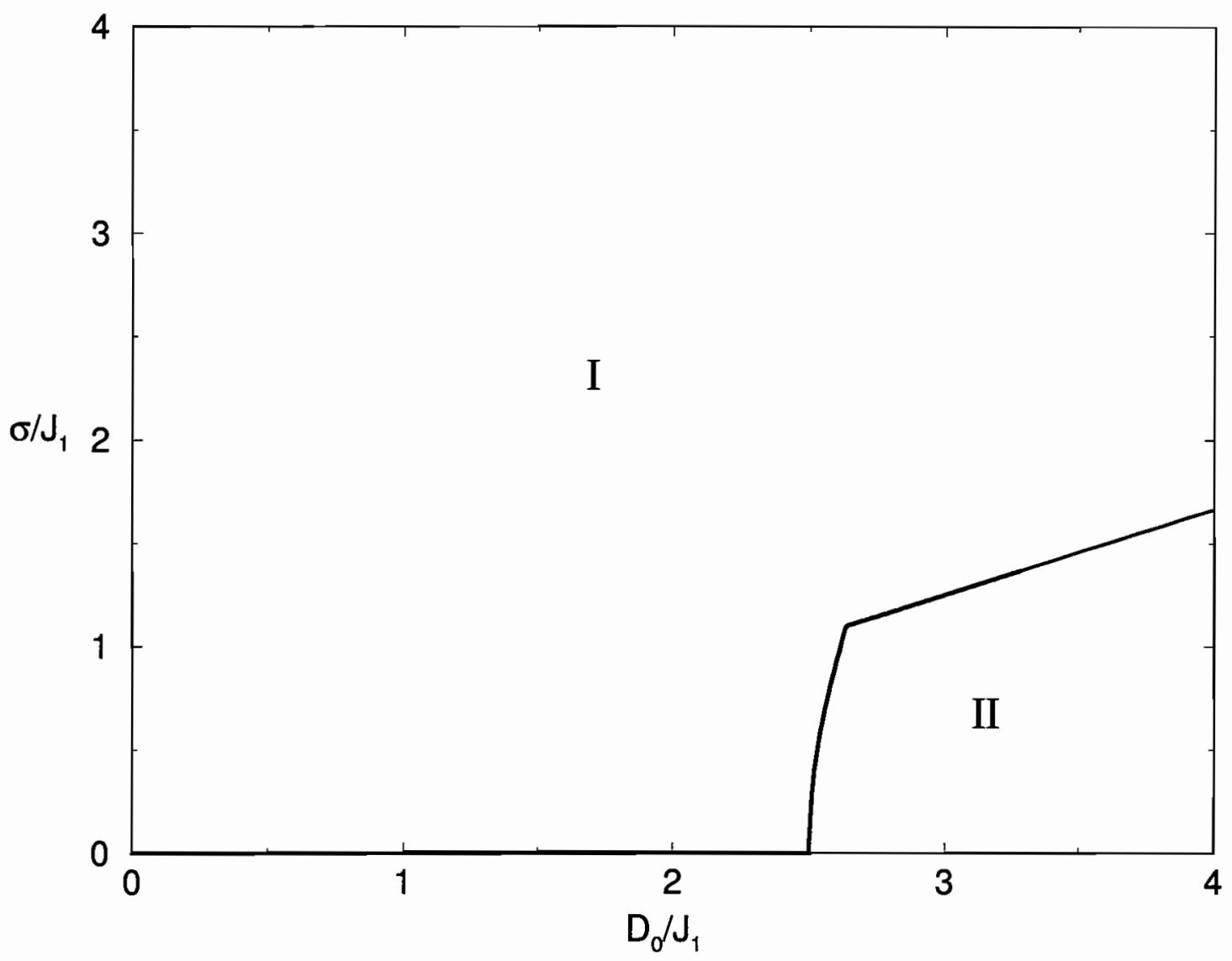

Figura 5.10: Linha que delimita as regiões II e I nas quais, respectivamente, existem e não existem pontos tricríticos. 


\subsection{Diagramas de fases para $T>0$}

Para a distribuição de probabilidades gaussiana (5.1), as equações de campo médio (3.19) e (3.20) ficam

$$
m_{z}=\frac{1}{\sigma \sqrt{2 \pi}} \int_{-\infty}^{+\infty} \frac{2 e^{-\beta D} \sinh \beta \eta_{z}}{1+2 e^{-\beta D} \cosh \beta \eta_{z}} \exp \left[-\frac{\left(D-D_{0}\right)^{2}}{2 \sigma^{2}}\right] d D
$$

e

$$
\eta_{z}=4 J_{0} m_{z}+J_{1}\left(m_{z+1}+m_{z-1}\right)+J_{2}\left(m_{z+2}+m_{z-2}\right) .
$$

Para o funcional da energia livre, novamente observamos que o primeiro termo no lado direito da equação (3.27) não depende de $m_{z}$. Portanto podemos ignorá-lo ao compararmos as energias livres das diferentes soluções. Logo, para o funcional da energia livre utilizado na região de temperaturas intermediárias vamos levar em conta apenas os termos que dependem de $m_{z}$,

$$
\begin{aligned}
N^{-3} \Phi= & -\frac{1}{N} \sum_{z} \int_{0}^{\eta_{z}} m_{z} d \eta_{z}+\frac{1}{2 N} \sum_{z}\left[4 J_{0} m_{z}^{2}+J_{1} m_{z}\left(m_{z+1}+m_{z-1}\right)\right. \\
& \left.+J_{2} m_{z}\left(m_{z+2}+m_{z-2}\right)\right]
\end{aligned}
$$

A partir das equações (5.12), (5.13) e (5.14) construímos os diagramas de fases do modelo no plano $k T / J_{1}$ versus $-J_{2} / J_{1}$, com $D_{0} / J_{1}$ e $\sigma / J_{1}$ fixos.

As fases moduladas são rotuladas apenas pelo seu número de onda seguido pela letra A (ausência de planos desordenados), B (presença de planos desordenados) ou $\mathrm{C}$ (fase sem qualquer simetria), sem qualquer tipo de extensão.

$\mathrm{Na}$ figura 5.11 apresentamos o diagrama de fases obtido para $D_{0} / J_{1}=1 \mathrm{e}$ $\sigma / J_{1}=0.5$. Notamos que o comportamento das fases moduladas é semelhante ao observado no diagrama de fases do modelo ANNNI usual com spin $\frac{1}{2}$. Com esse resultado concluímos que para o valor $\sigma / J_{1}=0.5$ a desordem do campo cristalino não exerce influência perceptível sobre o diagrama de fases. 


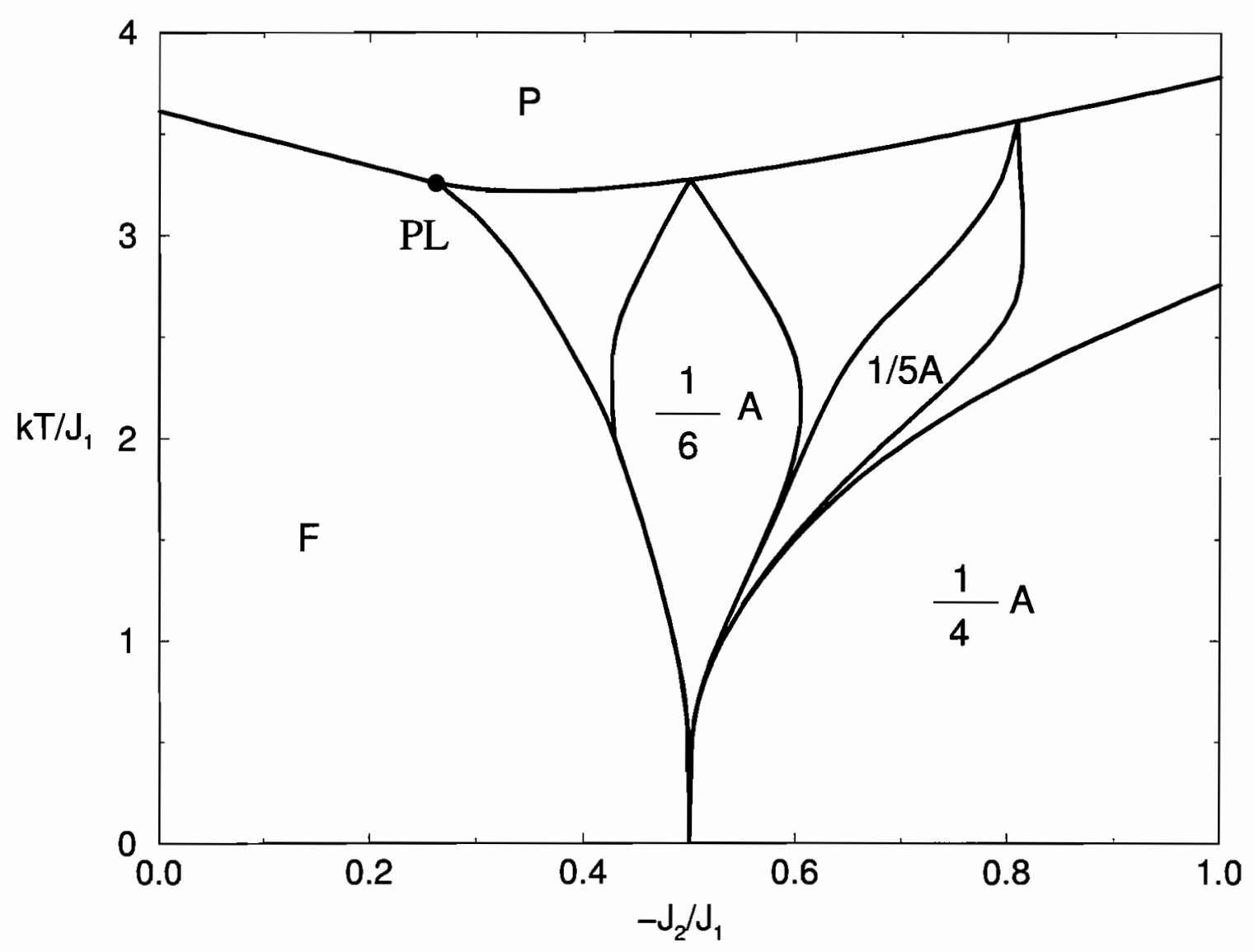

Figura 5.11: Diagrama de fases no plano $\left(k T / J_{1}\right)$ versus $-J_{2} / J_{1}$ para $D_{0} / J_{1}=1$ e $\sigma / J_{1}=$ 0.5 . As frações são os números de onda das fases moduladas. A linha crítica, separando a fase paramagnética $(\mathrm{P})$ da região ordenada, encontra-se com a linha de primeira ordem, separando a fase ferromagnética $(\mathrm{F})$ da região modulada, no ponto de Lifshitz (PL).

Na figura 5.12 mostramos o diagrama de fases obtido para $D_{0} / J_{1}=2.2$ e $\sigma / J_{1}=0.5$. Comparando esse diagrama com aquele obtido no caso de um campo cristalino constante (figura 4.20), não observamos influência perceptível da desordem sobre o comportamento das fases moduladas para $\sigma / J_{1}=0.5$.

Finalmente, na figura 5.13 apresentamos o diagrama de fases obtido para $D_{0} / J_{1}=2.8$ e $\sigma / J_{1}=1.5$. Para esses valores de $D_{0} / J_{1}$ e $\sigma / J_{1}$ notamos que o diagrama de fases torna-se muito complexo. Existe uma porção da linha crí- 


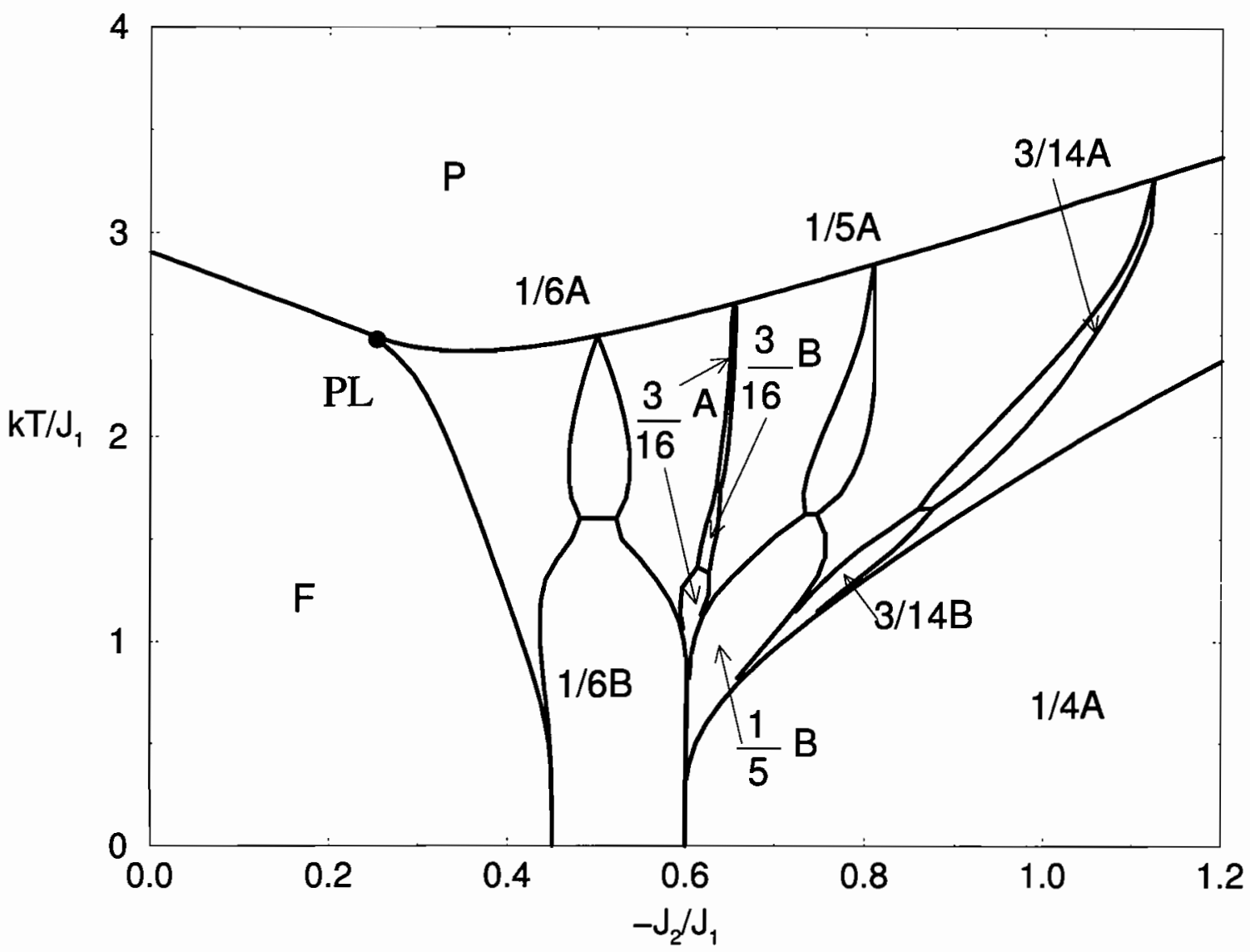

Figura 5.12: Diagrama de fases no plano $k T / J_{1}$ versus $-J_{2} / J_{1}$ para $D_{0} / J_{1}=2.2 \mathrm{e} \sigma / J_{1}=$ 0.5 . As frações são os números de onda das fases moduladas. A linha crítica, separando a fase paramagnética $(\mathrm{P})$ da região ordenada, encontra-se com a linha de primeira ordem, separando a fase ferromagnética (F) da região modulada, no ponto de Lifshitz (PL).

tica em temperaturas mais baixas que é desestabilizada pelo surgimento das fases moduladas (linha tracejada na figura 5.13). Dentro da região modulada e da fase ferromagnética $(\mathrm{F})$, a porção tracejada da linha crítica não existe, embora tenha sido incluída na figura 5.13. Acreditamos que nos pontos onde a linha crítica é desestabilizada surjam pontos bicríticos. No entanto, a localização precisa dos pontos bicríticos (caso realmente existam) torna-se muito difícil devido à complexidade dos diagramas de fases e porque seria necessário 
considerar-se fases de períodos extremamente longos.

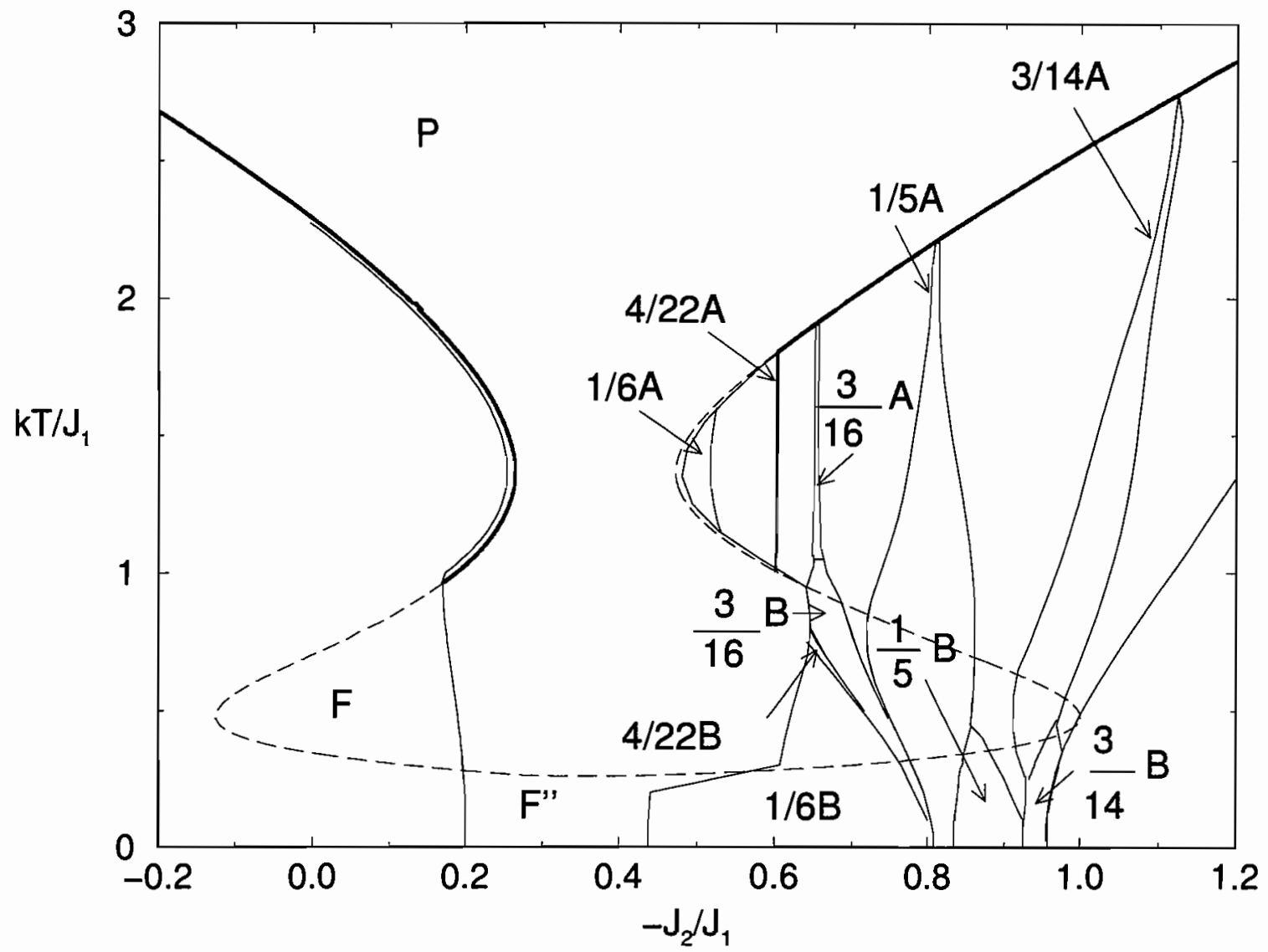

Figura 5.13: Diagrama de fases no plano $k T / J_{1}$ versus $-J_{2} / J_{1}$ para $D_{0} / J_{1}=2.8$ e $\sigma / J_{1}=$ 1.5. As frações são os números de onda das fases moduladas. A linha crítica é representada pela linha contínua grossa, ao passo que as transições de primeira ordem são representadas pelas linhas finas. A linha tracejada corresponde à porção da linha crítica desestabilizada pelo surgimento das fases moduladas. 


\section{Capítulo 6}

\section{Considerações finais}

Neste trabalho, estudamos os efeitos da desordem do campo cristalino sobre os diagramas de fases do modelo ANNNI com spin 1.

No capítulo 2 realizamos um estudo de Monte Carlo do modelo no caso de um campo cristalino constante (sem desordem). Determinamos os diferentes ordenamentos a partir da análise de Fourier do perfil da magnetização. Pudemos identificar dois ordenamentos distintos da fase comensurável de período 6 (fase 1/6): em um deles, existem planos desordenados na configuração da fase $(1 / 6 \mathrm{~B})$, ao passo que no outro ordenamento, tais planos estão ausentes $(1 / 6 \mathrm{~A})$. Ambas as fases 1/6A e 1/6B haviam sido encontradas anteriormente na aproximação de campo médio do modelo (Cadorin e Yokoi, 1998). Estudamos a distribuição de probabilidades da amplitude e da fase do harmônico principal. Em particular, a grande flutuação observada para a fase do harmônico principal próximo à transição entre as fases 1/6A e 1/6B pode ser interpretada como consequência de uma energia de ancoramento muito baixa na região de transição. Nossos resultados confirmam explicitamente a aplicabilidade da aproximação de campo médio no caso em que uma fase parcialmente desordenada está presente e fornecem credibilidade aos resultados de campo médio 
obtidos no caso em que as interações intraplanares $J_{0}$ não são muito pequenas em comparação às interações interplanares $J_{1}$.

No capítulo 3 iniciamos o estudo de campo médio do modelo com desordem do campo cristalino. Para uma distribuição de probabilidades genérica da anisotropia, obtivemos o funcional da energia livre e as equações de campo médio, assim como expressões para as linhas críticas e pontos tricríticos do modelo.

No capítulo 4 consideramos uma distribuição de probabilidades do campo cristalino delta-bimodal, com valor médio $D_{0}$ e desvio padrão $\sigma$. Assim, o modelo puro (sem desordem) corresponde a $\sigma=0$. Obtivemos diagramas de fases tanto em $T=0$ quanto em $T>0$ e investigamos os efeitos da desordem comparando nossos resultados, obtidos para $\sigma>0$, com resultados anteriores obtidos para $\sigma=0$ (Cadorin e Yokoi, 1998). No caso em que a desordem é fraca $\left(\sigma / J_{1} \approx 1\right)$, observamos que não há efeitos significativos sobre os diagramas de fases. Entretanto, conforme a desordem aumenta, os pontos tricríticos são desestabilizados e tornam-se ausentes do diagrama de fases em valores suficientemente altos do desvio padrão $\sigma$. Para investigarmos como a desordem afeta as fases moduladas, obtivemos numericamente o diagrama de fases do modelo em $T=0$. Para $\sigma=0$, a única fase modulada presente em $T=0$ é a fase $1 / 6 \mathrm{~B}$. Entretanto, para $\sigma>0$, observamos que outras fases moduladas tornam-se estáveis em $T=0$, dando origem a regiões moduladas complexas. Na região de temperaturas intermediárias dos diagramas de fases, nossos resultados numéricos mostraram que as fases com planos desordenados ocorrem em temperaturas mais baixas com o aumento de $\sigma$. Para valores de 
$\sigma$ suficientemente altos, as fases com planos desordenados tornam-se ausentes dos diagramas de fases. Para desordem ainda mais intensa, observamos que o diagrama de fases do modelo torna-se muito semelhante ao do modelo ANNNI com spin 1/2. Para verificarmos esse resultado numérico, realizamos um estudo de campo médio do modelo ANNNI com spin $1 / 2$ e diluição de sítios. Nessa aproximação, mostramos que existe o mapeamento $k T / J_{1} \rightarrow p k T / J_{1}, m_{z} \rightarrow$ $p m_{z}$ entre o modelo ANNNI com spin 1/2 e a sua versão com diluição de sítios, sendo $p$ a probabilidade de ocupação de um sítio. Ainda na aproximaçã̃o de campo médio, mostramos que o modelo ANNNI com spin $1 / 2$ e diluição de sítios corresponde ao limite $\sigma / J_{1} \rightarrow \infty$ do modelo ANNNI com spin 1 e campo cristalino delta-bimodal, em concordância com os resultados numéricos obtidos para valores de $\sigma$ mais altos.

No capítulo 5 estudamos o modelo na aproximação de campo médio e considerando uma distribuição de probabilidades do campo cristalino gaussiana, com valor médio $D_{0}$ e desvio padrão $\sigma$. Na região de altas temperaturas dos diagramas de fases nossos resultados mostraram que os pontos tricríticos são desestabilizados com o aumento da desordem. Também observamos que existem pontos tricríticos instáveis para certos valores de $D_{0}$ e $\sigma$. Esse fato indica a possibilidade de existência de pontos bicríticos e de pontos críticos terminais nos diagramas de fases. Nos diagramas de fases obtidos numericamente para $T=0$, observamos a existência de pontos críticos e a possibilidade de fases comensuráveis com períodos longos e fases incomensuráveis, diferentemente do caso de anisotropia delta-bimodal, no qual encontramos apenas períodos $Q \leq 14$. Finalmente, exploramos numericamente a região de temperaturas 
intermediárias dos diagramas de fases e observamos que, dependendo dos valores de $D_{0}$ e $\sigma$, a linha crítica pode ser desestabilizada pelas fases moduladas, possibilitando o surgimento de pontos bicríticos e pontos críticos terminais. 


\section{Apêndice A}

\section{Expansão de Landau para uma distribuição de probabilidades genérica do campo cristalino}

Neste apêndice desenvolvemos a expansão de Landau do funcional da energia livre (3.44) em potências do parâmetro de ordem do modelo, $\left|m_{q_{c}}\right|$. Usando a identidade

$$
\sum_{z} e^{i Q z}=N \Delta(Q)
$$

onde

$$
\Delta(Q)=\sum_{m=-\infty}^{+\infty} \delta_{Q+2 \pi m, 0}
$$

$\mathrm{e}$

$$
Q=\frac{2 \pi}{N} k \quad(k \text { inteiro }),
$$

o funcional (3.44) fica escrito na forma

$$
N^{-3} \Phi=-k T\left\langle\ln \left(1+2 e^{-\beta D}\right)\right\rangle_{c}+S_{2}+\frac{b_{3}}{4} S_{4}+\frac{b_{5}}{6} S_{6},
$$

onde

$$
\begin{gathered}
S_{2}=\frac{1}{2} \sum_{q_{1}, q_{2}}\left[b_{1}-J\left(q_{1}\right)\right] m_{q_{1}} m_{q_{2}} \Delta\left(q_{1}+q_{2}\right), \\
S_{n}=\sum_{q_{1}, q_{2}, \cdots, q_{n}} m_{q_{1}} m_{q_{2}} \cdots m_{q_{n}} \Delta\left(q_{1}+q_{2}+\cdots+q_{n}\right), \quad(n>2) .
\end{gathered}
$$


Para prosseguirmos a expansão, vamos supor que próximo à linha crítica a fase modulada é bem representada por uma magnetização por camada senoidal com um certo número de onda $q_{c}$. Vamos também supor que os harmônicos superiores são apenas correções que se tornam mais importantes à medida em que nos afastamos da linha crítica, penetrando na fase modulada (Yokoi et al., 1981). Também estamos supondo que os harmônicos superiores têm a forma

$$
\left|m_{n q_{c}}\right| \sim\left|m_{q_{c}}\right|^{n}
$$

Assim, consideramos a amplitude do harmônico principal $\left|m_{q_{c}}\right|$ como o parâmetro de ordem distinguindo as fases ordenada $\left(\left|m_{q_{c}}\right| \neq 0\right)$ e desordenada $\left(\left|m_{q_{c}}\right|=0\right)$. Até o quinto harmônico, a soma $S_{2}$ é

$$
\begin{aligned}
S_{2}= & {\left[b_{1}-J\left(q_{c}\right)\right]\left|m_{q_{c}}\right|^{2}+\left[b_{1}-J\left(2 q_{c}\right)\right]\left|m_{2 q_{c}}\right|^{2}+\left[b_{1}-J\left(3 q_{c}\right)\right]\left|m_{3 q_{c}}\right|^{2} } \\
& +\left[b_{1}-J\left(4 q_{c}\right)\right]\left|m_{4 q_{c}}\right|^{2}+\left[b_{1}-J\left(5 q_{c}\right)\right]\left|m_{5 q_{c}}\right|^{2}
\end{aligned}
$$

$\mathrm{Na}$ soma $S_{4}$ mantivemos termos até ordem $\left|m_{q_{c}}\right|^{6}$, e os termos em ordem dominante até o quinto harmônico. Observamos que os termos de Umklapp (correspondendo aos valores de $Q$ satisfazendo a soma (A.2) para $m \neq 0$ ) são termos de ordem superior e portanto ficam ausentes na soma. Logo,

$$
\begin{aligned}
S_{4}= & 6\left|m_{q_{c}}\right|^{4}+6\left|m_{2 q_{c}}\right|^{4}+4\left(m_{3 q_{c}} m_{q_{c}}^{*^{3}}+m_{3 q_{c}}^{*} m_{q_{c}}^{3}\right) \\
& +12\left(m_{4 q_{c}} m_{2 q_{c}}^{*} m_{q_{c}}^{*^{2}}+m_{4 q_{c}}^{*} m_{2 q_{c}} m_{q_{c}}^{2}+m_{5 q_{c}} m_{q_{c}}^{*} m_{2 q_{c}}^{*^{2}}\right. \\
& \left.+m_{5 q_{c}}^{*} m_{q_{c}} m_{2 q_{c}}^{2}+m_{5 q_{c}} m_{3 q_{c}}^{*} m_{q_{c}}^{*^{2}}+m_{5 q_{c}}^{*} m_{3 q_{c}} m_{q_{c}}^{2}\right)= \\
= & 6\left|m_{q_{c}}\right|^{4}+8 \operatorname{Re}\left\{m_{3 q_{c}}^{*} m_{q_{c}}^{3}\right\}+24\left(\operatorname { R e } \left\{m_{4 q_{c}}^{*} m_{2 q_{c}} m_{q_{c}}^{2}+m_{5 q_{c}}^{*} m_{q_{c}} m_{2 q_{c}}^{2}\right.\right. \\
& \left.+m_{5 q_{c}}^{*} m_{3 q_{c}} m_{q_{c}}^{2}\right\}+6\left|m_{2 q_{c}}\right|^{4}
\end{aligned}
$$

onde $\operatorname{Re}\{\cdots\}$ indica a parte real. Analogamente, obtivemos para $S_{6}$

$$
S_{6}=20\left(\left|m_{q_{c}}\right|^{6}+\left|m_{2 q_{c}}\right|^{6}\right)+30\left(m_{3 q_{c}}\left|m_{q_{c}}\right|^{2} m_{q_{c}}^{*^{3}}+m_{3 q_{c}}^{*}\left|m_{q_{c}}\right|^{2} m_{q_{c}}^{3}\right)
$$




$$
\begin{aligned}
& +120\left(m_{4 q_{c}}\left|m_{q_{c}}\right|^{2} m_{q_{c}}^{*^{2}} m_{2 q_{c}}^{*}+m_{4 q_{c}}^{*}\left|m_{q_{c}}\right|^{2} m_{q_{c}}^{2} m_{2 q_{c}}\right) \\
& +6\left(m_{5 q_{c}} m_{q_{c}}^{*^{5}}+m_{5 q_{c}}^{*} m_{q_{c}}^{5}\right) \\
& =20\left(\left|m_{q_{c}}\right|^{6}+\left|m_{2 q_{c}}\right|^{6}\right)+60 \operatorname{Re}\left\{m_{3 q_{c}}^{*}\left|m_{q_{c}}\right|^{2} m_{q_{c}}^{3}\right\}+12 \operatorname{Re}\left\{m_{5 q_{c}}^{*} m_{q_{c}}^{5}\right\} \\
& +240 \operatorname{Re}\left\{m_{4 q_{c}}^{*}\left|m_{q_{c}}\right|^{2} m_{q_{c}}^{2} m_{2 q_{c}}\right\} .
\end{aligned}
$$

Substituindo as equações (A.8), (A.9) and (A.10) no funcional (A.4) obtivemos

$$
\begin{aligned}
N^{-3} \Phi= & -k T\left\langle\ln \left(1+2 e^{-\beta D}\right)\right\rangle_{c}+\left[b_{1}-J\left(q_{c}\right)\right]\left|m_{q_{c}}\right|^{2}+\left[b_{1}-J\left(2 q_{c}\right)\right]\left|m_{2 q_{c}}\right|^{2} \\
+ & {\left[b_{1}-J\left(3 q_{c}\right)\right]\left|m_{3 q_{c}}\right|^{2}+\left[b_{1}-J\left(4 q_{c}\right)\right]\left|m_{4 q_{c}}\right|^{2}+\left[b_{1}-J\left(5 q_{c}\right)\right]\left|m_{5 q_{c}}\right|^{2} } \\
+ & {\left[b_{1}-J\left(6 q_{c}\right)\right]\left|m_{6 q_{c}}\right|^{2}+\frac{b_{3}}{4}\left[6\left|m_{q_{c}}\right|^{4}+6\left|m_{2 q_{c}}\right|^{4}+8 \operatorname{Re}\left\{m_{3 q_{c}}^{*} m_{q_{c}}^{3}\right\}\right.} \\
& \left.+24\left(\operatorname{Re}\left\{m_{4 q_{c}}^{*} m_{2 q_{c}} m_{q_{c}}^{2}+m_{5 q_{c}}^{*} m_{q_{c}} m_{2 q_{c}}^{2}+m_{5 q_{c}}^{*} m_{3 q_{c}} m_{q_{c}}^{2}\right\}\right)\right] \\
& +\frac{10}{3} b_{5}\left(\left|m_{q_{c}}\right|^{6}+\left|m_{2 q_{c}}\right|^{6}\right)+10 b_{5} \operatorname{Re}\left\{m_{3 q_{c}}^{*}\left|m_{q_{c}}\right|^{2} m_{q_{c}}^{3}\right\} \\
& +40 b_{5} \operatorname{Re}\left\{m_{4 q_{c}}^{*}\left|m_{q_{c}}\right|^{2} m_{q_{c}}^{2} m_{2 q_{c}}\right\}+2 b_{5} \operatorname{Re}\left\{m_{5 q_{c}}^{*} m_{q_{c}}^{5}\right\} .
\end{aligned}
$$

Para eliminarmos os termos $m_{n q_{c}}(n>1)$ e escrever a expansão apenas em termos do parâmetro de ordem $\left|m_{q_{c}}\right|$, usamos o fato de que $\Phi$ deve ser estacionário em relação a $m_{n q_{c}}$. Assim, levando em conta apenas termos em ordem dominante envolvendo o segundo harmônico, obtivemos para $\left|m_{2 q_{c}}\right|$

$$
\frac{\partial\left(N^{-3} \Phi\right)}{\partial\left|m_{2 q_{c}}\right|}=2\left[b_{1}-J\left(2 q_{c}\right)\right]\left|m_{2 q_{c}}\right|+6 b_{3}\left|m_{2 q_{c}}\right|^{3}=0,
$$

o que implica

$$
\left|m_{2 q_{c}}\right|=0 \text {. }
$$

Analogamente, todos os harmônicos pares se anulam, isto é,

$$
\left|m_{n q_{c}}\right|=0 \quad \text { para } n=2,4,6,8, \cdots
$$


O próximo harmônico que devemos calcular é $m_{3 q_{c}}$. Antes de impor a condição de ponto estacionário para $\Phi$ relativamente a esse harmônico, vamos determinar o seu ângulo de fase, $\phi_{3 q_{c}}$. Com a definição

$$
m_{n q_{c}}=\left|m_{n q_{c}}\right| e^{i \phi_{n q_{c}}}
$$

podemos escrever

$$
b_{3} \operatorname{Re}\left\{m_{3 q_{c}}^{*} m_{q_{c}}^{3}\right\}=b_{3}\left|m_{3 q_{c}}\right|\left|m_{q_{c}}^{3}\right| \cos \left(-\phi_{3 q_{c}}+3 \phi_{q_{c}}\right)
$$

Assim, para minimizarmos o funcional da energia livre $\Phi$, devemos ter

$$
\phi_{3 q_{c}}= \begin{cases}3 \phi_{q_{c}}+\pi & , \text { se } b_{3}>0 \\ 3 \phi_{q_{c}} & , \text { se } b_{3}<0\end{cases}
$$

o que implica

$$
b_{3} \operatorname{Re}\left\{m_{3 q_{c}}^{*} m_{q_{c}}^{3}\right\}=-\left|b_{3}\right|\left|m_{3 q_{c}}\right|\left|m_{q_{c}}\right|^{3} .
$$

Com as equações (A.14) e (A.18), o funcional da energia livre (A.11) fica

$$
\begin{aligned}
N^{-3} \Phi= & -k T\left\langle\ln \left(1+2 e^{-\beta D}\right)\right\rangle_{c}+\left[b_{1}-J\left(q_{c}\right)\right]\left|m_{q_{c}}\right|^{2}+\left[b_{1}-J\left(3 q_{c}\right)\right]\left|m_{3 q_{c}}\right|^{2} \\
& +10 b_{5} \operatorname{Re}\left\{m_{3 q_{c}}^{*}\left|m_{q_{c}}\right|^{2} m_{q_{c}}^{3}\right\}+\left[b_{1}-J\left(5 q_{c}\right)\right]\left|m_{5 q_{c}}\right|^{2}+\frac{3}{2} b_{3}\left|m_{q_{c}}\right|^{4} \\
& -2\left|b_{3}\right|\left|m_{3 q_{c}}\right|\left|m_{q_{c}}\right|^{3}+6 b_{3} \operatorname{Re}\left\{m_{5 q_{c}}^{*} m_{3 q_{c}} m_{q_{c}}^{2}\right\} \\
& +2 b_{5} \operatorname{Re}\left\{m_{5 q_{c}}^{*} m_{q_{c}}^{5}\right\}+\frac{10}{3} b_{5}\left|m_{q_{c}}\right|^{6}
\end{aligned}
$$

A seguir, impusemos a condição de ponto estacionário para $\Phi$,

$$
\frac{\partial\left(N^{-3} \Phi\right)}{\partial\left|m_{3 q_{c}}\right|}=2\left[b_{1}-J\left(3 q_{c}\right)\right]\left|m_{3 q_{c}}\right|-2\left|b_{3}\right|\left|m_{q_{c}}\right|^{3}=0,
$$

considerando apenas termos de ordem dominante envolvendo o terceiro harmônico. Com isso, da equação (A.20) obtemos

$$
\left|m_{3 q_{c}}\right|=\left|b_{3}\right|\left[b_{1}-J\left(3 q_{c}\right)\right]^{-1}\left|m_{q_{c}}\right|^{3} .
$$


Analogamente, para calcular $m_{5 q_{c}}$ consideramos

$$
6 b_{3} \operatorname{Re}\left\{m_{5 q_{c}}^{*} m_{3 q_{c}} m_{q_{c}}^{2}\right\}+2 b_{5} \operatorname{Re}\left\{m_{5 q_{c}}^{*} m_{q_{c}}^{5}\right\}
$$

o qual, usando a notação (A.15), fica

$2 b_{5}\left|m_{5 q_{c}}\right|\left|m_{q_{c}}\right|^{5} \cos \left(-\phi_{5 q_{c}}+5 \phi_{q_{c}}\right)+6 b_{3}\left|m_{5 q_{c}}\right|\left|m_{3 q_{c}}\right|\left|m_{q_{c}}\right|^{2} \cos \left(-\phi_{5 q_{c}}+\phi_{3 q_{c}}+2 \phi_{q_{c}}\right)$.

Usando o resultado (A.17) podemos ainda escrever o resultado acima sob a forma

$$
\left\{2 b_{5}-6\left|b_{3}\right|^{2}\left[b_{1}-J\left(3 q_{c}\right)\right]^{-1}\right\}\left|m_{5 q_{c}}\right|\left|m_{q_{c}}\right|^{5} \cos \left(-\phi_{5 q_{c}}+5 \phi_{q_{c}}\right) .
$$

Logo, para minimizar a energia livre, devemos ter

$$
\phi_{5 q_{c}}=5 \phi_{q_{c}}+\pi \quad, \text { se } 2 b_{5}-6\left|b_{3}\right|^{2}\left[b_{1}-J\left(3 q_{c}\right)\right]^{-1}>0
$$

e

$$
\phi_{5 q_{c}}=5 \phi_{q_{c}} \quad, \text { se } 2 b_{5}-6\left|b_{3}\right|^{2}\left[b_{1}-J\left(3 q_{c}\right)\right]^{-1}<0 \text {. }
$$

Assim,

$$
\begin{aligned}
& b_{3} \operatorname{Re}\left\{m_{5 q_{c}}^{*} m_{3 q_{c}} m_{q_{c}}^{2}\right\}+2 b_{5} \operatorname{Re}\left\{m_{5 q_{c}}^{*} m_{q_{c}}^{5}\right\}= \\
= & -\left.\left.2\left|b_{5}-3\right| b_{3}\right|^{2}\left[b_{1}-J\left(3 q_{c}\right)\right]^{-1}|| m_{5 q_{c}}|| m_{q_{c}}\right|^{5}
\end{aligned}
$$

Com o resultado (A.27), o funcional (A.19) fica

$$
\begin{aligned}
N^{-3} \Phi= & -k T\left\langle\ln \left(1+2 e^{-\beta D}\right)\right\rangle_{c}+\left[b_{1}-J\left(q_{c}\right)\right]\left|m_{q_{c}}\right|^{2}+\left[b_{1}-J\left(3 q_{c}\right)\right]\left|m_{3 q_{c}}\right|^{2} \\
& +\left[b_{1}-J\left(5 q_{c}\right)\right]\left|m_{5 q_{c}}\right|^{2}+\frac{3}{2} b_{3}\left|m_{q_{c}}\right|^{4}-2\left|b_{3}\right|\left|m_{3 q_{c}}\right|\left|m_{q_{c}}^{3}\right| \\
& +10 b_{5} \operatorname{Re}\left\{m_{3 q_{c}}^{*}\left|m_{q_{c}}\right|^{2} m_{q_{c}}^{3}\right\}-\left.\left.2\left|b_{5}-3\right| b_{3}\right|^{2}\left[b_{1}-J\left(3 q_{c}\right)\right]^{-1}|| m_{5 q_{c}}|| m_{q_{c}}\right|^{5} \\
& +\frac{10}{3} b_{5}\left|m_{q_{c}}\right|^{6} .
\end{aligned}
$$


Da condição de ponto estacionário para $\Phi$

$$
\begin{aligned}
\frac{\partial\left(N^{-3} \Phi\right)}{\partial\left|m_{5 q_{c}}\right|} & =2\left[b_{1}-J\left(5 q_{c}\right)\right]\left|m_{5 q_{c}}\right| \\
& =-\left.\left.2\left|b_{5}-3\right| b_{3}\right|^{2}\left[b_{1}-J\left(3 q_{c}\right)\right]^{-1}|| m_{q_{c}}\right|^{5}=0
\end{aligned}
$$

resulta

$$
\left|m_{5 q_{c}}\right|=\left[b_{1}-J\left(5 q_{c}\right)\right]^{-1}\left\{\left.\left|b_{5}-3\right| b_{3}\right|^{2}\left[b_{1}-J\left(3 q_{c}\right)\right]^{-1} \mid\right\}\left|m_{q_{c}}\right|^{5} .
$$

Os resultados (A.21) e (A.30) confirmam nossa hipótese inicial (A.7), isto é,

$$
\left|m_{n q_{c}}\right| \sim\left|m_{q_{c}}\right|^{n} \quad \text { para } n=3,5,7,9, \cdots
$$

Uma vez que nosso objetivo é obter a expansão do funcional da energia livre até termos da ordem $\left|m_{q_{c}}\right|^{6}$, levamos em conta apenas o terceiro e o quinto harmônicos, desde que harmônicos ímpares superiores estão acima da ordem de aproximação desejada. Portanto, substituindo os resultados (A.21) e (A.30) em (A.28), obtivemos o funcional da energia livre em sua forma final

$$
N^{-3} \Phi=\Phi_{0}+C_{2}\left|m_{q_{c}}\right|^{2}+C_{4}\left|m_{q_{c}}\right|^{4}+C_{6}\left|m_{q_{c}}\right|^{6}+\cdots,
$$

onde

$$
\begin{aligned}
\Phi_{0} & =-k T\left\langle\ln \left(1+2 e^{-\beta D}\right)\right\rangle_{c}, \\
C_{2} & =b_{1}-J\left(q_{c}\right) \\
C_{4} & =\frac{3}{2} b_{3} \\
C_{6} & =\frac{10}{3} b_{5}-b_{3}^{2}\left[b_{1}-J\left(3 q_{c}\right)\right]^{-1} .
\end{aligned}
$$




\section{Apêndice B}

\section{Determinação do ponto crítico em $T=0$ para uma distribuição gaussiana do campo cristalino}

Neste apêndice desenvolvemos os cálculos para a obtenção do ponto crítico encontrado nos diagramas de fases do modelo em $T=0$, no caso de uma distribuição gaussiana do campo cristalino. Para determinar o ponto crítico, vamos fazer $m_{z}=m$, e $\eta_{z}=\eta$ para todo $z$, uma vez que as fases envolvidas são ferromagnéticas $\left(\mathrm{F}_{\text {e }} \mathrm{F}^{\prime \prime}\right)$. Além disso, como o funcional da energia livre (5.4) é uma função par de $m$, podemos considerar $m>0$. Por outro lado, supondo que a quantidade $\left(4 J_{0}+2 J_{1}+2 J_{2}\right)>0$, se $m>0$ então $\eta>0$. Com isso, as equações de campo médio (5.2) e (3.30) ficam

$$
m=\frac{1}{2} \operatorname{erfc}\left(\frac{D_{0}-\eta}{\sigma \sqrt{2}}\right),
$$

e

$$
m=\frac{\eta}{6 J_{1}+2 J_{2}} .
$$

O sistema formado pelas equações (B.1) e (B.2) admite 3 soluções para $\sigma<\sigma_{c}$ (figura B.1a), e apenas uma solução para $\sigma \geq \sigma_{c}$ (figuras B.1b e B.1c). Para $\sigma<\sigma_{c}$, na figura B.1a observamos que existem dois valores de $\eta\left(\eta_{+}\right.$e $\left.\eta_{-}\right)$ 
satisfazendo a equação

$$
\frac{d}{d \eta}\left[\frac{1}{2} \operatorname{erfc}\left(\frac{D_{0}-\eta}{\sigma \sqrt{2}}\right)\right]=\frac{1}{\sigma \sqrt{2 \pi}} e^{\frac{-\left(D_{0}-\eta\right)^{2}}{2 \sigma^{2}}}=\frac{1}{6 J_{1}+2 J_{2}}
$$

sendo

$$
\eta_{ \pm}=D_{0} \pm \sigma \sqrt{2}\left[\ln \left(\frac{6 J_{1}+2 J_{2}}{\sigma \sqrt{2 \pi}}\right)\right]^{\frac{1}{2}} .
$$

No ponto crítico (figura B.1b), $\eta_{+}=\eta_{-}=\eta=D_{0}$. Com isso, das equações (B.1), (B.2) e (B.4) obtemos as coordenadas do ponto crítico

$$
\begin{aligned}
-\frac{J_{2}}{J_{1}} & =3-\frac{D_{0}}{J_{1}} \\
\frac{\sigma}{J_{1}} & =\frac{D_{0}}{J_{1}} \sqrt{\frac{2}{\pi}}
\end{aligned}
$$

e a magnetização $m=1 / 2$ como a única solução possível para $\sigma \geq \sigma_{c}$ (figuras B.1b e B.1c). Em princípio, poderíamos encontrar pontos críticos nos diagramas de fases em $T=0$ para todos os valores de $D_{0} / J_{1}$. No entanto, a fase $F^{\prime \prime}$ torna-se paramagnética para $\sigma=0$. Por outro lado, a fase paramagnética existe no diagrama de fases com $\sigma=0$ apenas nas regiões III e IV (figura 4.4). Portanto, em $T=0$ existem pontos críticos nos diagramas de fases para $D_{0} / J_{1}>2.666 \cdots$. 

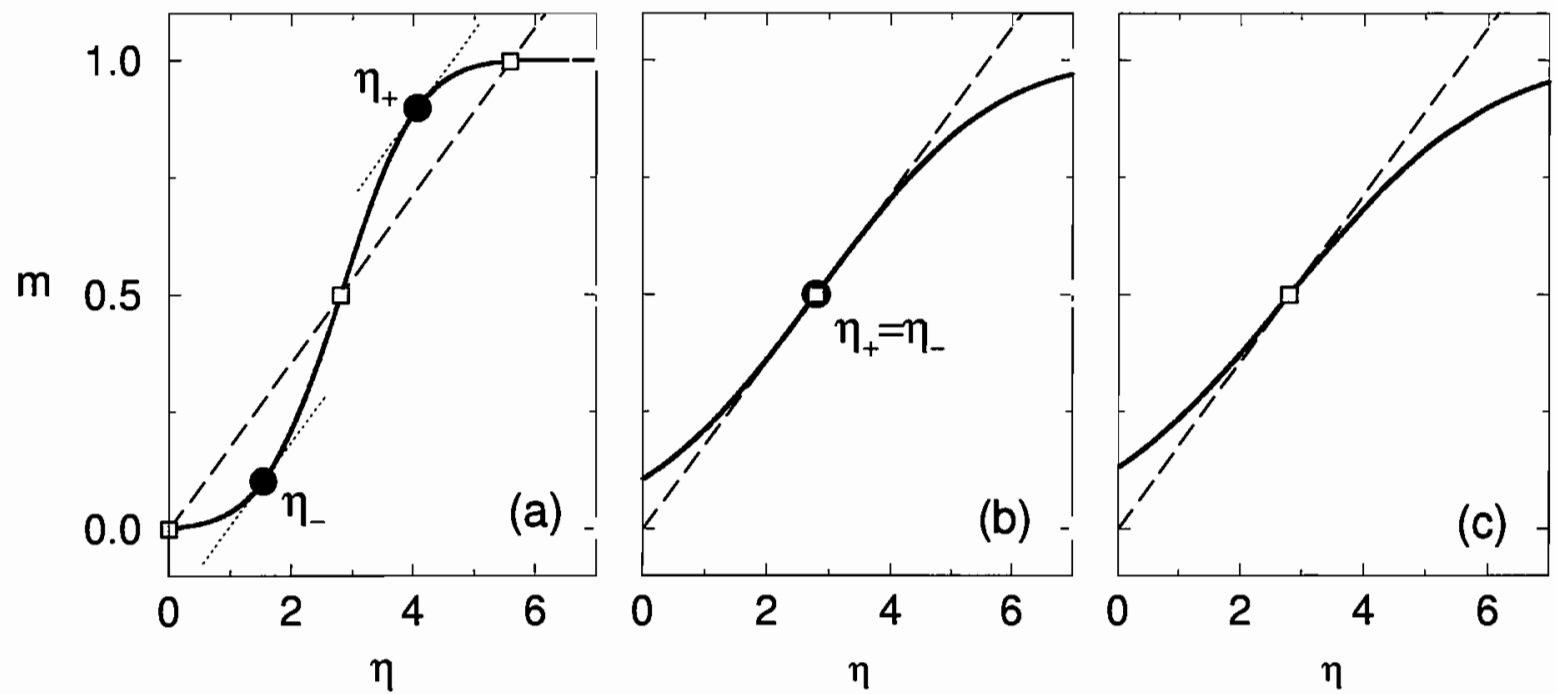

Figura B.1: Solução gráfica do sistema formado pelas equações (B.1) (linha contínua) e (B.2) (linha tracejada) para diferentes valores de $\sigma$. (a) Situação em que $\sigma<\sigma_{c}$. Existem três possíveis soluções para $m$ e $\eta$, representadas pelos quadrados. Os círculos correspondem aos valores $\eta_{ \pm}$satisfazendo a equação (B.4). Para $\sigma<\sigma_{c}, \eta_{+} \neq \eta_{-}$. As linhas pontilhadas são paralelas à linha tracejada e indicam que nos pontos $\eta_{+}$e $\eta_{-}$as derivadas das equações (B.1) e (B.2) são iguais. (b) Ponto crítico $\left(\sigma=\sigma_{c}\right)$. Existe apenas uma solução para as equações (B.1) e (B.2) dada por $m=0.5$ e $\eta=D_{0}$. Nesse caso, $\eta=\eta_{+}=\eta_{-}=D_{0}$. Com isso, os quadrados e os círculos colapsam no ponto $\left(m=0.5, \eta=D_{0}\right)$. (c) Caso $\sigma>\sigma_{c}$. Existe apenas a solução $m=0.5$ e $\eta=D_{0}$ para as equações (B.1) e (B.2) (quadrado). A equação (B.4) não admite solução para $\sigma>\sigma_{c}$ e por isso os círculos estão ausentes. 


\section{Bibliografia}

Alves Jr., N., e Yokoi, C. S. O. (2000). "Spin Pair Correlation of the ANNNI

Chain in a Field". Braz. J. Phys., 34(4), 667-670.

Aubry, S. (1978). Solitons and condensed matter. Berlin: Springer.

Bak, P. (1982). "Commensurate phases, incommensurate phases and the devil's staircase". Rep. Prog. Phys., 45, 587-629.

Bak, P., e Boehm, J. von. (1980). "Ising model with solitons, phasons and the Devil's staircase". Phys. Rev. B, 21(11), 5297-5308.

Bak, P., Coppersmith, S., Shapir, Y., Fishman, S., e Yeomans, J. (1985). "The instability of long-range commensurate phases in the presence of quenched impurities". J. Phys. C, 18(20), 3911-3918.

Barbosa Filho, F. F. (1996). Efeitos do campo magnético e do campo cristalino no diagrama de fases do modelo ANNNI. Unpublished doctoral dissertation, Universidade de São Paulo.

Barreto, M., e Yeomans, J. M. (1985). "The axial Ising model with third neighbour interactions: low temperature expansion". J. Phys. A, 134, $84-122$. 
Becerra, C. C., Shapira, Y., Oliveira Jr., N. F. de, e Chang, T. S. (1980). "Lifshitz Point in MnP". Phys. Rev. Lett., 44(25), 1692-1695.

Benyoussef, A., Biaz, T., Saber, M., e Touzani, M. (1987). "The spin-1 Ising model with a random crystal field: the mean-field solution". J. Phys. C, 20, 5349-5354.

Bhattacharyya, N., e Dasgupta, S. (1991). "Statistical mechanics of the 1D ferromagnetic ANNNI chain under an external field: revisited". J. Phys. $A$, 24, 3927-3934.

Binder, K., e Young, A. P. (1986). "Spin glasses: Experimental facts, theoretical concepts and open questions". Rev. Mod. Phys., 58(4), 801-976.

Blume, M. (1966). "Theory of the First-Order Magnetic Phase Change in uo. $_{2}$. Phys. Rev., 141(2), 517-524.

Boehm, J. von, e Bak, P. (1979). "Devil's Stairs and the CommensurateCommensurate Transitions in CeSb". Phys. Rev. Lett., 42(2), 122-125.

Bohr, J., Gibbs, D., Moncton, D. E., e Damico, K. L. (1986). "Spin slips and lattice modulations in holmium - a magnetic-x-ray scattering study". Physica A, 140(1-2), 349-358.

Borelli, M. E. S., e Carneiro, C. E. I. (1996). "Global mean-field phase diagram of the spin-1 Ising ferromagnet in a random crystal field". Physica A, 230(1-2), 249-256.

Cadorin, J. L., e Yokoi, C. S. O. (1993). "Mean-Field Axial Next-Nearest- 
Neighbor Ising Model with an Arbitrary Intralayer Interaction". Braz. J. Phys., 23, 382-386.

Cadorin, J. L., e Yokoi, C. S. O. (1998). "Spin-1 ANNNI model with crystalfield anisotropy". Physica A, 248, 176-184.

Callen, H. B. (1985). Thermodynamics and an Introduction to Thermostatistics (2nd ed.). New York: John Wiley \& Sons.

Capel, H. W. (1966). "On the possibility of first-order phase transitions in Ising systems of triplet ions with zero-field splitting". Physica (Utrecht), 32, 966-988.

Carneiro, C. E. I., Henriques, V. B., e Salinas, S. R. A. (1989). "Comment on the mean-field phase diagram of the spin-1 Ising model in a random crystal field". J. Phys.: Condensed Matter, 1, 3687-3689.

Carneiro, C. E. I., Henriques, V. B., e Salinas, S. R. A. (1990). "Meanfield phase diagram of the spin-1 Ising ferromagnet in a Gaussian random crystal field". J. Phys. A, 23, 3383-3388.

Comes, R., Shapiro, S. M., Shirane, G., Garito, A. F., e Heeger, A. J. (1975). "Neutron-scattering study of $38-\mathrm{k}$ and $54-\mathrm{k}$ phase-transitions in deuterated tetrathiafulvalene-tetracyanoquinodimethane (ttf-tcnq)". Phys. Rev. Lett., 35(22), 1518-1521.

Cooper, R. B. (1968). "Magnetic Properties of Rare Earth Metals". In F. Seitz, D. Turnbull, e H. Ehrenreich (Eds.), Solid state physics (Vol. 21). New York: Academic Press. (pp. 393-490) 
Cummins, H. Z. (1990). "Experimental studies of structurally incommensurate crystal phases". Phys. Rep., 185(5-6), 211-409.

Duxbury, P. M., e Selke, W. (1983). "Branching-processes in the ANNNI model". J. Phys. A, 16(18), L741-L744.

Elliott, R. J. (1961). "Phenomenological discussion of magnetic ordering in the heavy rare earth metals". Phys. Rev., 124(2), 346-353.

Elliott, R. J. (1972). Magnetic Properties of Rare Earth Metals. New York: Plenum.

Falk, H. (1970). "Inequalities of J. W. Gibbs". Am. J. Phys., 38(7), 858-869.

Felcher, G. P., Lander, G. H., Arai, T., Sinha, S. K., e Spedding, F. H. (1976). "Asphericity in magnetization distribution of holmium". Phys. Rev. B, 13(7), 3034-3045.

Fischer, J. E., e Thompson, T. E. (1978). "Graphite intercalation compounds". Phys. Today, 31(7), 36.

Fischer, K. H., e Hertz, J. A. (1993). Spin Glasses. Cambridge: Cambridge University Press.

Fisher, M. E., e Selke, W. (1980). "Infinitely many commensurate phases in a simple Ising-model". Phys. Rev. Lett., 44(23), 1502-1505.

Fishman, S., e Yeomans, J. (1985). "Can long-period phases remain stable in the presence of quenched impurities?". J. Phys. C, 18(4), 857-867. 
Gennes, P. G. de. (1974). The Physics of Liquid Crystals. Oxford: Oxford University Press.

Gibbs, D., Bohr, J., Axe, J. D., Moncton, D. E., e Damico, K. L. (1986). "Magnetic-structure of erbium". Phys. Rev. B, 34(11), 8182-8185.

Griffiths, R. B. (1990). "Frenkel-Kontorova models of commensurateincommensurate phase transitions". In H. van Beijeren (Ed.), Fundamental problems in statistical physics (Vol. VII). Amsterdam: Elsevier Science. (pp. 69-110)

Hornreich, R. M., Luban, M., e Shtrikman, S. (1975). "Critical behavior at the onset of k-space instability on the $\lambda$ line". Phys. Rev. Lett., 35(25), 1678-1681.

Houlrik, J. M., e Jessen, S. J. K. (1986). "Phase diagram of the three-state chiral clock model studied by Monte Carlo renormalization-group calculations". Phys. Rev. B, 34(1), 325-329.

Huse, D. A. (1981). "Simple 3-state model with infinitely many phases". Phys. Rev. B, 24(9), 5180-5194.

Iizumi, M., Axe, J. D., Shirane, G., e Shimaoka, K. (1977). "Structural phase transformation in $\mathrm{K}_{2} \mathrm{SeO}_{4}$ ". Phys. Rev. B, 15(9), 4392-4411.

Izyumov, Y. A. (1984). "The modulated or long-period magnetic-structures of crystals". Usp. Fiz. Nauk., 144(3), 439-474.

Kaplan, T. A. (1959). "Classical spin-configuration stability in the presence of competing exchange forces". Phys. Rev., 116(4), 888-889. 
Kasuya, T. (1956). "A Theory of Metallic and Antiferromagnetism on Zener's model". Prog. Theor. Phys., 16(1), 45-57.

Kawasaki, T. (1984). "Concentration Dependence of Modulated Phases in Diluted ANNNI models". Prog. Theor. Phys., 71(2), 246-255.

Kincaid, J. M., e Cohen, E. G. D. (1975). "Phase Diagrams of Liquid Helium Mixtures and Metamagnets: Experiment and Mean-Field Theory". Phys. Rep., 22(2), 57-143.

Koehler, W. C., Cable, J. W., Child, H. R., Wilkinson, M. K., e Wollan, E. O. (1966). "Antiferromagnetism in chromium alloys. I. Neutron diffraction". Phys. Rev. B, 151, 414.

Koehler, W. C., Cable, J. W., Child, H. R., Wilkinson, M. K., e Wollan, E. O. (1967). "Magnetic structures of holmium. II. Magnetization process". Phys. Rev. B, 158, 450 .

Metropolis, N., Rosenbluth, A. W., Rosenbluth, M. N., Teller, A. H., e Teller, E. (1953). "Equation of State Calculations by Fast Computing Machines". J. Chem. Phys., 21(6), 1087-1092.

Metropolis, N., e Ulam, S. (1949). "The Monte Carlo Method". J. Am. Stat. Assoc., 44(247), 335-341.

Mézard, M., Parisi, G., e Virasoro, M. A. (1987). Spin glass theory and beyond (Vol. 9). Singapore: World Scientific.

Micheletti, C., Seno, F., e Yeomans, J. M. (1995). "Upsilon point in a spin model". Phys. Rev. B, 52(6), 4353-4359. 
Moorjani, K., e Coey, J. M. D. (1984). Methods and Phenomena 6 - Magnetic Glasses. Amsterdam: Elsevier.

Morita, T., e Horiguchi, T. (1972). "Spin orderings of the one-dimensional Ising magnet with the nearest and next nearest neighbor interactions". Phys. Lett., 38A(4), 223-224.

Muraoka, Y., Oda, K., e Idogaki, T. (1999). "Ground state phase diagrams of $S=1$ ANNNI model with the single-ion anisotropy". J. Magn. Magn. Mater., 195, 156-167.

Mydosh, J. A. (1993). Spin glasses: an experimental introduction. London: Burgess Science Press.

Nagamiya, T. (1967). "Helical Spin Ordering-1 Theory of Helical Spin Configurations". In F. Seitz, D. Turnbull, e H. Ehrenreich (Eds.), Solid state physics (Vol. 20). New York: Academic Press. (pp. 305)

Nakanishi, K. (1989). "Nonlinear Effect on the Axial Next-Nearest-Neighbor Ising (ANNNI) Model: Application to CeSb". J. Phys. Soc. Jpn., 58(4), $1296-1306$.

Nakanishi, K. (1992). "Absence of Partially Disordered States in the Axial Next-Nearest-Neighbor Ising (ANNNI) model". J. Phys. Soc. Jpn., 61(8), 2901-2908.

Neubert, B., Pleimling, M., e Siems, R. (1998). "Transitions between phases with equal wavenumbers in a double Ising spin model. Application to betaine calcium chloride dihydrate". J. Phys. C, 10,6883-6896. 
Newman, M. E. J., e Barkema, G. T. (1999). Monte Carlo Methods in Statistical Physics. Oxford (NY): Clarendon Press.

Ostlund, S. (1981). "Incommensurate and commensurate phases in asymmetric clock models". Phys. Rev. B, 24(1), 398-405.

Ottinger, H. C. (1983). "Numerical-solution of the mean-field theory of the ANNNI-model in an external-field - a comparison of 2 methods". J. Phys. A, 16(7), 1483-1489.

Pechan, M. J., e Stassis, C. (1984). "Magnetic-structure of holmium". J. Appl. Phys., 55(6), 1900-1902.

Pokrovsky, V. L., e Uimin, G. V. (1982). "Phase-diagram of the ANNNI model in external magnetic-field - application to CeSb". J. Phys. C, 15(11), L353-L356.

Rasmussen, E. B., e Jessen, S. J. K. (1981). "Devil's staircase behavior of a simple spin model". Phys. Rev. B, 24(5), 2744-2750.

Redner, S., e Stanley, H. E. (1977). "The r-s model for magnetic systems with competing interactions: series expansions and some rigorous results". $J$. Phys. C, 10, 4765-4784.

Roeder, H., e Yeomans, J. (1985). "Annealed Defects in Long Period Structures". J. Phys. C, 18(7), L163-L168.

Rossat-Mignod, J., Burlet, P., Bartholin, H., Vogt, O., e Lagnier, R. (1980). "Specific heat analysis of the magnetic phase diagram of CeSb". J. Phys. C, 13, 6381-6389. 
Rossat-Mignod, J., Burlet, P., Villain, J., Bartholin, H., Tcheng-Si, W., Florence, D., e Vogt, O. (1977). "Phase Diagrams and Magnetic Structures of CeSb". Phys. Rev. B, 16(1), 440-461.

Rothaus, F., e Selke, W. (1993). "Comment on Absence of Partially Disordered States in the Axial Next-Nearest-Neighbor Ising (ANNNI) model". $J$. Phys. Soc. Jpn., 62(1), 378-379.

Ruderman, M. A., e Kittel, C. (1954). "Indirect Exchange Coupling of Nuclear Moments by Conduction Electrons". Phys. Rev., 96(1), 99-102.

Rujan, P., Selke, W., e Uimin, G. V. (1983). "Rectangular lattice gas model with competing interactions and the two-dimensional ANNNI model in a field". Z. Phys. B, 53, 221-231.

Sasaki, K. (1992). "Modulated Phases and Upsilon Points in a Spin Model with Helical Ordering". J. Stat. Phys., 68(5-6), 1013-1035.

Sato, A., e Matsubara, F. (1999). "Equilibrium properties of an axial nextnearest-neighbor Ising model in two dimensions". Phys. Rev. B, 60(14), 10316-10324.

Scholten, P. D., e King, D. R. (1996). "Monte Carlo study of the three dimensional chiral clock model". Phys. Rev. B, 53(6), 3359-3363.

Selke, W. (1978). "Monte Carlo Calculations Near a Uniaxial Lifshitz Point". Z. Phys. B, 29, 133-137.

Selke, W. (1988). "The ANNNI model - theoretical analysis and experimental application". Phys. Rep., 170, 213-264. 
Selke, W. (1992). Spatially modulated structures in systems with competing interactions (Vol. 15). New York: Academic Press. (Capítulo 1, pp. 1-72)

Selke, W., Barreto, M., e Yeomans, J. M. (1985). "Axial Ising model with third-neighbour interactions". J. Phys. C, 18, L393-L399.

Selke, W., e Duxbury, P. M. (1984). "The mean-field theory of the three dimensional ANNNI model". Z. Phys. B, 57, 49-58.

Selke, W., e Fisher, M. E. (1979). "Monte Carlo study of the spatially modulated phase in an Ising model". Phys. Rev. B, 20(1), 257-265.

Smith, J., e Yeomans, J. M. (1982). "Low-temperature analysis of the ANNNI model in a field". J. Phys. C, 15, L1053-L1057.

Smith, J., e Yeomans, J. M. (1983). "Phase diagram of the ANNNI model in a field using a low temperature series technique". J. Phys. C, 16, 5305-5320.

Stephens, P. W., Heiney, P. A., Birgeneau, R. J., Horn, P. M., Moncton, D. E., e Brown, G. S. (1984). "High-resolution X-ray scattering study of the commensurate-incommensurate transition of monolayer-Kr on graphite". Phys. Rev. B, 29(13), 3512-3532.

Stephenson, J. (1970a). "Ising Model with Antiferromagnetic Next-NearestNeighbor Couplings: Spin Correlations and Disorder Points". Phys. Rev. $B, \mathbf{1}(11), 4405-4409$.

Stephenson, J. (1970b). "Two one-dimensional Ising models with disorder points". Can. J. Phys., 48, 1724-1734. 
Stinchcombe, R. B. (1983). Dilute magnetism (Vol. 7). London: Academic Press. (Capítulo 3, pp. 151-280)

Szpilka, A. M., e Fisher, M. E. (1985). "Domain-wall interactions and spatially modulated phases". Phys. Rev. Lett., 57, 1044-1047.

Takeda, K., Konishi, K., Deguchi, H., Iwata, N., e Shigeoka, T. (1991). "Thermodynamical study of the magnetic transition in commensurate phases in $\mathrm{PrCo}_{2} \mathrm{Si}_{2}$ ". J. Magn. Magn. Mater., 104-107, 901-902.

Tamashiro, M. N., Yokoi, C. S. O., e Salinas, S. R. A. (1997). "Mean-field calculations for the Axial Next-Nearest-Neighbor Ising model in a random field". Phys. Rev. B, 56(13), 8204-8211.

Toulouse, G. (1977). "Theory of Frustration Effect in Spin-Glasses 1". Commun. Phys., 2(4), 115-119.

Uimin, G. V. (1984). "Low-temperature analysis of the ANNNI model in an external magnetic field: cascades of phase transitions, complete devil's staircases". J. Stat. Phys., 34, 1-34.

Villain, J. (1959). "The structure of magnetic substances". J. Phys. Chem. Solids, 11(3-4), 303-309.

Yamada, Y., Hoshino, S., e Shibuya, I. (1963). "Phase transition in $\mathrm{NaNO}_{2}$ ". J. Phys. Soc. Jpn., 18, 1594-1603.

Yeomans, J. M. (1988). Solid State Physics (Vol. 41). San Diego (CA): Academic Press. (pp. 151-200) 
Yokoi, C. S. O. (1991). "Asymmetric phases in the mean-field theory of the Axial Next-Nearest-Neighbor Ising model". Phys. Rev. B, 43(10), 84878490 .

Yokoi, C. S. O., Coutinho-Filho, M. D., e Salinas, S. R. A. (1981). "Ising model with competing axial interactions in the presence of a field: A mean-field treatment". Phys. Rev. B, 24(7), 4047-4061.

Yokoi, C. S. O., Coutinho-Filho, M. D., e Salinas, S. R. A. (1985). "Isingmodel with competing axial interactions in the presence of a field - a renormalization-group treatment". Phys. Rev. B, 31(7), 4502-4507.

Yokoi, C. S. O., Tang, L.-H., e Chou, W. (1988). "Ground state of the onedimensional chiral XY model in a field". Phys. Rev. B, 37(4), 2173-2198.

Yoshimori, A. (1959). "A new type of antiferromagnetic structure in the rutile type crystal". J. Phys. Soc. Jpn., 14(6), 807-821.

Yosida, K. (1957). "Magnetic Properties of Cu-Mn Alloys". Phys. Rev., 106(5), 893-898.

Young, A. P. (editor). (1997). Spin glass theory and beyond (Vol. 12). Singapore: World Scientific.

Ziěba, A., Becerra, C., Fjellvåg, H., Oliveira Jr., N. F., e Kjekshus, A. (1992). " $\mathrm{Mn}_{0.9} \mathrm{Co}_{0.1} \mathrm{P}$ in an external field: Lifshitz point and irreversibility behavior of disordered incommensurate phases". Phys. Rev. B, 46, 3380-3390.

Ziěba, A., Becerra, C., Oliveira Jr., N. F., Fjellvåg, H., e Kjekshus, A. (1992). 
" $\mathrm{Mn}_{0.9} \mathrm{Co}_{0.1} \mathrm{P}$ in field parallel to hard direction: phase diagram and irreversibility of CONE phase". J. Magn. Magn. Mater., 104-107, 71-72.

Ziěba, A., Fjellvåg, H., e Kjekshus, A. (1988). "Magnetic phase diagrams and properties of transition metal substituted". J. Phys. Chem. Solids, $\mathbf{4 9}$, $1087-1090$. 